\title{
Clinical Evidence for Thermometric Parameters to Guide Hyperthermia Treatment
}

\author{
Adela Ademaj ${ }^{1,2}$, Danai P. Veltsista ${ }^{3}$, Pirus Ghadjar ${ }^{3}$, Dietmar Marder ${ }^{1}\left(\mathbb{D}\right.$, Eva Oberacker $^{3} \mathbb{D}$, Oliver J. Ott $^{4,5} \mathbb{D}^{\mathbb{D}}$, \\ Peter Wust ${ }^{3}$, Emsad Puric ${ }^{1}$, Roger A. Hälg 1,6 , Susanne Rogers ${ }^{1}$, Stephan Bodis 1,7, Rainer Fietkau 4,5, \\ Hans Crezee ${ }^{8}(\mathbb{D})$ and Oliver Riesterer ${ }^{1, *(\mathbb{D}}$
}

check for

updates

Citation: Ademaj, A.; Veltsista, D.P.; Ghadjar, P.; Marder, D.; Oberacker, E.; Ott, O.J.; Wust, P.; Puric, E.; Hälg,

R.A.; Rogers, S.; et al. Clinical

Evidence for Thermometric

Parameters to Guide Hyperthermia

Treatment. Cancers 2022, 14, 625.

https://doi.org/10.3390/

cancers 14030625

Academic Editor: David Wong

Received: 30 November 2021

Accepted: 19 January 2022

Published: 26 January 2022

Publisher's Note: MDPI stays neutral with regard to jurisdictional claims in published maps and institutional affiliations.

Copyright: (c) 2022 by the authors. Licensee MDPI, Basel, Switzerland. This article is an open access article distributed under the terms and conditions of the Creative Commons Attribution (CC BY) license (https:// creativecommons.org/licenses/by/ $4.0 /)$.
1 Center for Radiation Oncology KSA-KSB, Cantonal Hospital Aarau, 5001 Aarau, Switzerland; adela.ademaj@ksa.ch (A.A.); dietmar.marder@ksa.ch (D.M.); emsad.puric@ksa.ch (E.P.); roger.haelg@ksa.ch (R.A.H.); susanne.rogers@ksa.ch (S.R.); s.bodis@bluewin.ch (S.B.)

2 Doctoral Clinical Science Program, Medical Faculty, University of Zurich, 8032 Zürich, Switzerland

3 Department Radiation Oncology, Charité-Universitätsmedizin Berlin, Corporate Member of Freie Universität Berlin and Humboldt-Universität zu Berlin, 13353 Berlin, Germany; paraskevi-danai.veltsista@charite.de (D.P.V.); pirus.ghadjar@charite.de (P.G.); eva.oberacker@charite.de (E.O.); peter.wust@charite.de (P.W.)

4 Department of Radiation Oncology, Universitätsklinikum Erlangen, 91054 Erlangen, Germany; oliver.ott@uk-erlangen.de (O.J.O.); rainer.fietkau@uk-erlangen.de (R.F.)

5 Comprehensive Cancer Center Erlangen-EMN, 91054 Erlangen, Germany

6 Institute of Physics, Science Faculty, University of Zurich, 8057 Zurich, Switzerland

7 Department of Radiation Oncology, University Hospital Zurich, University of Zurich, 8091 Zurich, Switzerland

8 Department of Radiation Oncology, Amsterdam UMC, University of Amsterdam, Cancer Center Amsterdam, 1105 AZ Amsterdam, The Netherlands; h.crezee@amsterdamumc.nl

* Correspondence: oliver.riesterer@ksa.ch; Tel.: +41-62838-4249

Simple Summary: Hyperthermia (HT) is a promising therapeutic option for multiple cancer entities as it has the potential to increase the cytotoxicity of radiotherapy (RT) and chemotherapy (CT). Thermometric parameters of HT are considered to have potential as predictive factors of treatment response. So far, only limited data about the prognostic and predictive role of thermometric parameters are available. In this review, we investigate the existing clinical evidence regarding the correlation of thermometric parameters and cancer response in clinical studies in which patients were treated with HT in combination with RT and/or CT. Some studies show that thermometric parameters correlate with treatment response, indicating their potential significance for treatment guidance. Thus, the establishment of specific thermometric parameters might pave the way towards a better standardization of HT treatment protocols.

Abstract: Hyperthermia (HT) is a cancer treatment modality which targets malignant tissues by heating to $40-43{ }^{\circ} \mathrm{C}$. In addition to its direct antitumor effects, HT potently sensitizes the tumor to radiotherapy (RT) and chemotherapy $(\mathrm{CT})$, thereby enabling complete eradication of some tumor entities as shown in randomized clinical trials. Despite the proven efficacy of HT in combination with classic cancer treatments, there are limited international standards for the delivery of HT in the clinical setting. Consequently, there is a large variability in reported data on thermometric parameters, including the temperature obtained from multiple reference points, heating duration, thermal dose, time interval, and sequence between HT and other treatment modalities. Evidence from some clinical trials indicates that thermal dose, which correlates with heating time and temperature achieved, could be used as a predictive marker for treatment efficacy in future studies. Similarly, other thermometric parameters when chosen optimally are associated with increased antitumor efficacy. This review summarizes the existing clinical evidence for the prognostic and predictive role of the most important thermometric parameters to guide the combined treatment of RT and CT with HT. In conclusion, we call for the standardization of thermometric parameters and stress the importance for their validation in future prospective clinical studies. 
Keywords: hyperthermia; thermometric parameters; preclinical data; clinical evidence

\section{Introduction}

Hyperthermia (HT) is a clinical treatment for cancer which extraneously and intrinsically heats malignant cells to a temperature of $40-43{ }^{\circ} \mathrm{C}$ for a suitable period of time [1,2]. Heat delivered to tumor tissues can act as a cytotoxic or sensitizing agent to enhance their remission or at least regression by utilizing several biological mechanisms and pleiotropic effects when combined with other conventional cancer treatment techniques, such as radiotherapy (RT) and/or chemotherapy (CT).

The biological effects of HT, which all favor its use in combination with RT and CT, include direct cytotoxicity, radiosensitization, chemosensitization, and immune modulation. HT-induced cell lethality is predominantly a result of conformational changes and the destabilization of macromolecule structures including the disruptions in cell metabolism, inhibition of DNA repair, and triggering of cellular apoptotic pathways [3-6]. The direct HT-induced cell lethality is known to be intrinsically tumor-selective for hypoxic cells [7]. During heating, enhanced blood perfusion in tumor tissues influences the radiosensitizing and chemosensitizing effects of HT by increasing the tumor oxygenation level and local concentration of CT drugs respectively $[4,8,9]$. Radiosensitization and chemosensitization effects, as well as the inhibition of DNA synthesis and repair, on the molecular level depend on the aggregation of proteins produced by HT-induced denaturation [10]. Moreover, protein unfolding and the intracellular accumulation of proteins trigger molecular chaperones including the heat shock proteins (HSPs) [11]. The release of HSPs and other "immune activating signals" underly the inflammatory and immunogenic responses to HT in combination with RT and/or CT and can promote anti-tumor immunity [12-14]. Exploiting molecular and physiological mechanisms evoked by HT can improve the efficacy of RT and CT. Therefore, HT in cancer treatment is used mainly within the framework of multimodal treatment strategies $[3,8]$.

Multiple preclinical studies have been designed to unravel the relationship between biological mechanisms induced by HT and thermometric parameters as predictors of tumor response [15-20]. The parameters investigated in these studies include the temperature achieved during HT [6,15], heating duration, thermal dose [21], time interval between HT and the other treatment modality [15,22,23], the number of HT sessions [24], and the sequence of treatment modality $[15,25,26]$. All of these parameters were shown to influence the extent to which HT enhances the effect of RT or CT using cellular assays and in vivo models. In addition to thermometric parameters, the treatment parameters of RT and $\mathrm{CT}$, such as total radiation dose, number of RT fractions, type of chemotherapeutic drug and the number of $\mathrm{CT}$ cycles, prescribed for a specific clinical indication, also play a significant role in attaining a therapeutic window with synergistic effects when combined with HT $[25,27,28]$.

The effectiveness of HT combined with RT and/or CT has been investigated in many clinical studies with different tumor types. Unfortunately, to date, there is no consensus on HT delivery when combined with these cancer treatment modalities, resulting in substantial heterogeneity of the HT treatment protocols applied. Any comparison of these studies in terms of outcome should be made with caution in view of this heterogeneity in HT protocols. A good understanding of thermometric parameters and their interpretation is mandatory in this regard. However, there is inconclusive clinical evidence about the relationship of thermometric parameters with both tumor and normal tissue responses to $\mathrm{HT}$ in combination with RT and/or CT. The reason for this is that thermometric parameters are inconsistently reported or analyzed in prospective clinical studies and the retrospective analyses are conflicting. For instance, minimum tumor temperature was identified as a prognostic factor in a few studies [29-31]. However, another study showed that different metrics such as temperature achieved in $90 \%\left(\mathrm{~T}_{90}\right), 50 \%\left(\mathrm{~T}_{50}\right)$, and $10 \%\left(\mathrm{~T}_{10}\right)$ in the target 
volume were more strongly correlated with cancer response than minimum achieved temperature [32]. Furthermore, a short time interval between HT and RT was shown to significantly predict treatment outcome in retrospective analyses of cervical cancer patients [22]. However, conflicting results have been also reported [33] which may be attributed to differences in time interval and tumor temperature achieved, and in patient population [34]. Thermal dose has been successfully tested in several clinical trials as a predictor of tumor response to combined RT and HT treatment [35-42]. These did not result in established thresholds for thermal dose for treating different cancer sites, even though European Society for Hyperthermic Oncology (ESHO) guidelines recommend superficial HT maintains $\mathrm{T}_{50} \geq 41{ }^{\circ} \mathrm{C}$ and $\mathrm{T}_{90} \geq 40{ }^{\circ} \mathrm{C}$ [43]. The concept of a relationship between thermometric parameters with treatment outcome is highly attractive because it could improve the understanding of tumor-specific mechanisms of interaction between HT and RT and/or CT. Defining thermometric parameters is therefore important for a meaningful clinical evaluation of HT treatment outcomes when combined with RT and/or CT.

A limited amount of clinical information is available about the effect of thermometric parameters on treatment response. Increasing awareness of the importance of such parameters on the efficacy of HT combined with other cancer treatments is important, and thus these parameters should be evaluated and reported routinely. Achieving the defined thermometric parameters during HT treatment would further increase the effectiveness of biological mechanisms when combined with RT and/or CT. Future prospective clinical studies should include description of all relevant thermometric parameters to pave the way towards the proper analysis and standardization of thermometric parameters for each clinical indication treated with HT in combination with RT and/or CT.

This work summarizes the evidence underlying thermometric parameters as predictors of treatment outcomes as reported in clinical studies using HT in combination with RT and/or CT for treating different cancer types and emphasizes the need for reference thermometric parameters to improve HT efficacy. For completeness, the findings pertaining to thermometric parameters from preclinical studies are also discussed, to provide comprehensive information about their significance and underlying mechanisms.

\section{Materials and Methods}

\subsection{Data Sources and Search Strategies}

The literature search included databases of clinicaltrials.gov and pubmed.ncbi.nlm. nih.gov from March to September 2021 and randomized prospective and retrospective clinical studies with specific criteria were identified. The search terms were hyperthermia, cancer treatment, randomized clinical studies, prospective clinical studies, and retrospective clinical studies. Those terms were used mainly to search for the title and abstract. We also found articles which were recommended, suggested, or sent to us on the internet. Additionally, we handsearched the reference lists of the most relevant clinical studies and review articles.

\subsection{Inclusion and Exclusion Criteria of Clinical Studies}

This non-systematic review included randomized, prospective, and retrospective clinical studies that recruited patients with cancer who were treated with HT and RT and/or CT. The data from randomized trials are only from the patient group which received HT in combination with either RT and/or CT. Data from the non-HT arm were not extracted.

The main inclusion criteria was the use of either electromagnetic, radiative, or capacitive HT systems, independent of cancer type. Another criterion was more than 10 patients recruited in prospective and retrospective studies. Retrospective studies were only included if analysis of thermometric parameters for HT in combination with RT had been performed.

Clinical studies which used the thermal ablation technique, interstitial-based $/$ modulated electro HT techniques, interstitial RT techniques, high intensity focused ultrasound (HIFU) HT, whole body HT, and studies in pediatric patients were not included in this review. Pilot and feasibility studies were also excluded. 


\subsection{Data Extraction and List of Variables Included}

The data extracted from the clinical studies contained the following information:

- First author of the study

- Study design: prospective or retrospective

- $\quad$ RT treatment data: total dose, number of fractionations

- CT treatment data: drug and concentration prescribed, number of cycles

- Thermometric parameters

- Reported clinical endpoints

- Reported relationship between thermometric parameters and clinical endpoint

\subsection{A Summary of HT Techniques}

The clinical studies included in this review administered HT using externally applied power with electromagnetic-based techniques, such as radiofrequency, microwave, or infrared. These techniques differ with regard to their application to treat superficial or deep-seated tumors, as summarized elsewhere [44].

For superficial tumors, the electromagnetic radiative and capacitive systems are the those used in the clinical trials included in this review. The superficial HT techniques and their application are explained in detail elsewhere [43]. The radiative and capacitive systems differ in the way they are applied in the clinic. A study showed that for superficial cancers, the radiative HT system performs better than capacitive systems in terms of temperature distribution [45]. The commercially available radiative superficial systems are the BSD-500 device (Pyrexar Medical, Salt Lake, UT, USA), the ALBA ON4000 (Alba Hyperthermia, Rome, Italy) and contact flexible microwave applicators (SRPC Istok, Fryazino, Moscow region, Russia). Thermotron RF8 (Yamamoto Vinita Co, Osaka, Japan), Oncotherm (Oncotherm Kft., Budapest, Hungary) and Celsius TCS (Celsius42 GmbH, Cologne, Germany) are examples of commercial capacitive systems used for superficial tumors.

Different HT techniques with unique specifications, characteristics, and limitations are used to treat deep-seated tumors [46]. The ESHO guidelines provide information as to how and when a specific particular HT device should be used to treat deep-seated tumors [46,47]. The radiative HT systems for deep-seated tumors used in clinical trials are the BSD-2000 device (Pyrexar Medical, Salt Lake, UT, USA), the ALBA 4D (Alba Hyperthermia, Rome, Italy), and the Synergo RITE (Medical Enterprises Europe B.V., Amstelveen, The Netherlands), and capacitive systems are Oncotherm (Oncotherm Kft., Budapest, Hungary), Celsius TCS (Celsius $42 \mathrm{GmbH}$, Cologne, Germany), and Thermotron RF8 (Yamamoto Vinita Co, Osaka, Japan). Another simulation study showed a difference in heating patterns between radiative and capacitive HT for deep-seated tumors [48]. The radiative technique yields more favorable simulated temperature distributions for deep-seated tumors than the capacitive technique.

\subsection{Definition of Thermometric Parameters}

In this work, the thermometric parameters were extracted from the selected perspective and retrospective clinical studies. The definitions of these parameters are listed in Table 1. 
Table 1. Definition of thermometric parameters.

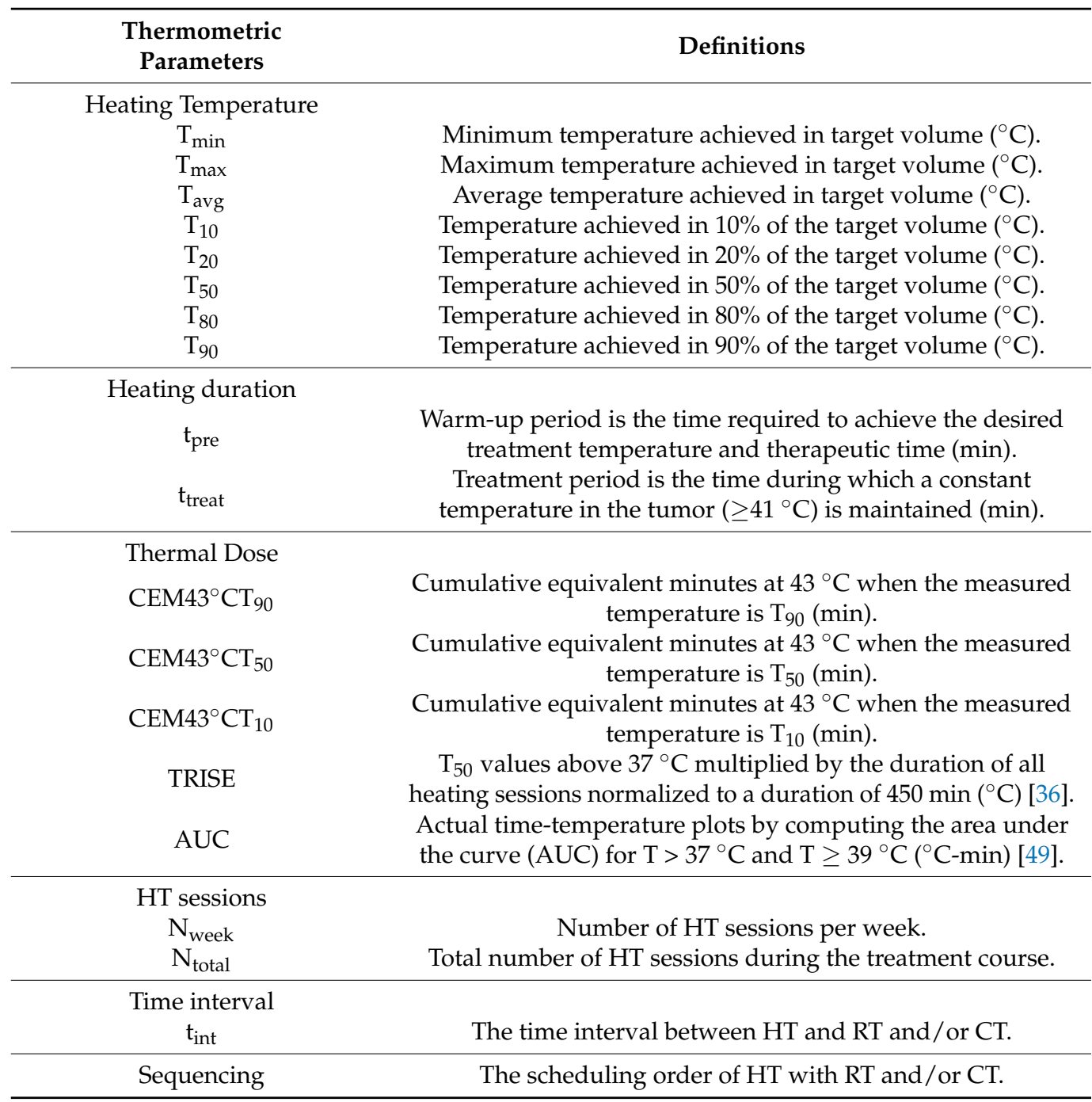

Temperature measurements in the target volume or surrounding tissue are crucial for assessing treatment quality and are represented by temperature metrics. During a HT session, the temperature is usually monitored and recorded using high resistance thermistor probes, fiber optic temperature probes or thermocouples by invasively placing the probes in the target volume or in the vicinity of the target volume $[43,46,50]$. The ESHO guidelines recommend that after the definition of the tumor volume as a planning target volume, a target point should be defined where the probe is positioned intraluminally or intratumorally [46]. In addition, the guidelines strongly suggest keeping a record of thermometry measurement points within or close to the tumor sites [43]. After completion of the HT session, recorded temperature data during $t_{\text {treat }}$ are evaluated by computing temperature metrics. For instance, $T_{\max }$ is calculated as the maximum temperature value recorded in the target volume (Table 1 ). $\mathrm{T}_{10}$, another maximum temperature metric, is computed as the temperature value received by $10 \%$ of the target volume [32]. Similarly, the other temperature metrics listed in Table 1 are computed. In current practice, the thermometric parameters and thermal dose are computed by software integrated in the HT systems or using thermal analysis tools such as RHyThM [51].

To illustrate how temperature, $t_{\text {pre }}$ and $t_{\text {treat }}$ terms are measured in clinical practice, Figure 1 shows the temperature and heating duration parameters of a patient treated with HT in the radiation oncology center at Cantonal Hospital Aarau (KSA) using BSD-500 system (BSD Medical Corporation, Salt Lake City, UT, USA). 


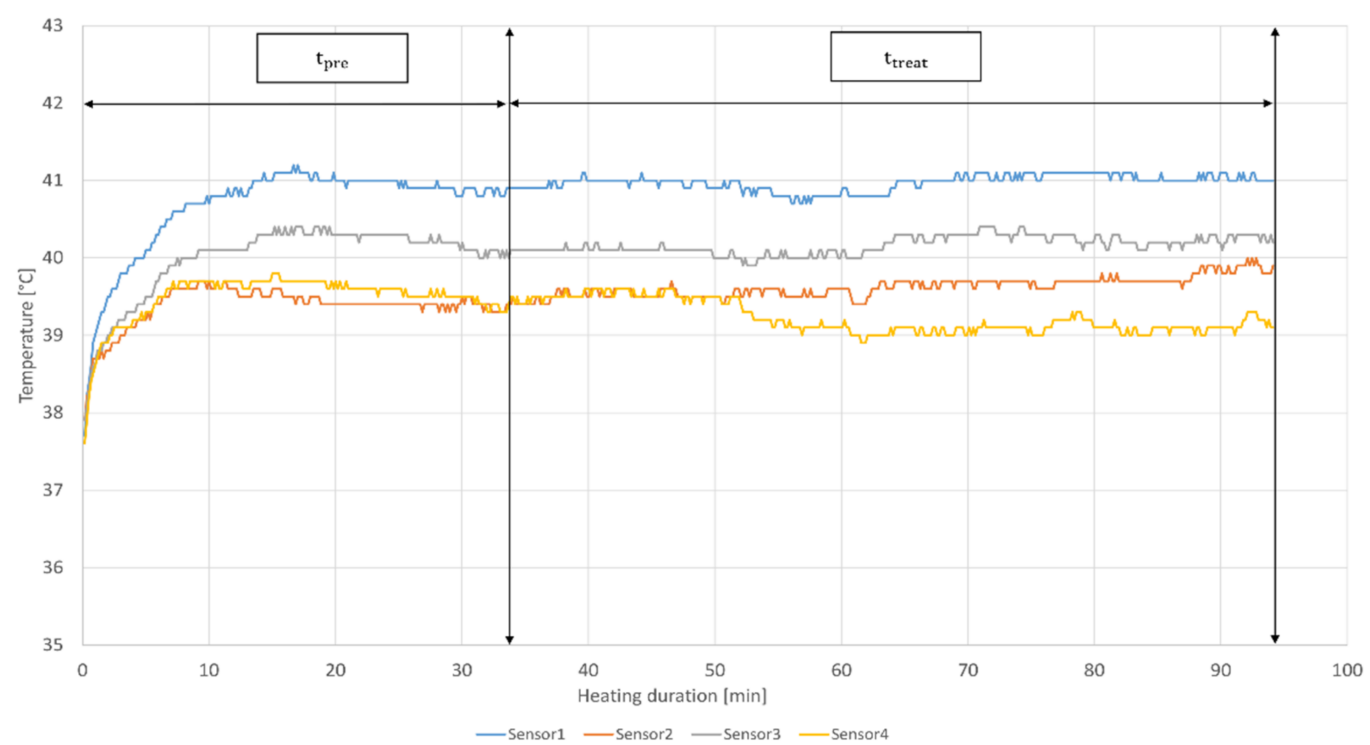

Figure 1. Recorded treatment data of a single HT session for a breast cancer patient. Temperature in ${ }^{\circ} \mathrm{C}$ and heating duration in minutes are measured non-invasively using four sensors located in close proximity to the tumor tissue. $t_{\text {pre }}$ and $t_{\text {treat }}$ of 33 and 60 min respectively according to the KSA clinical protocol are indicated.

The temperature metrics and thermal doses can be also computed by using the data from Figure 1. A decade ago, a new thermal dose entitled "TRISE" was proposed by Franckena et al. [36]. However, this parameter has not yet been evaluated in experimental studies. Another newly proposed thermal dose parameter is the area under the curve (AUC) [49]. In contrast to CEM $43^{\circ} \mathrm{C}$ and TRISE, AUC is computed without any prior assumptions by summating AUC for the entire treatment session, including $t_{\text {pre }}$ and $t_{\text {treat }}$. Similarly to TRISE, AUC has not yet been investigated in preclinical studies. Another parameter related to HT used in this review is thermal enhancement ratio (TER), defined as 'the ratio between RT dose required to achieve a specific endpoint and RT dose to achieve the same endpoint in combination with $\mathrm{HT}^{\prime}$ [52].

\section{Evidence for Predictive Values of Thermometric Parameters in Preclinical Studies \\ 3.1. Heating Temperature}

The responsiveness of a tumor to HT is determined by different heat-induced mechanisms at the cellular level. The oxygenation rate is affected by temperature, as a higher rate was reported at $41-41.5^{\circ} \mathrm{C}$ in comparison to higher temperature (at $43^{\circ} \mathrm{C}$ ) in rodent tumors, human tumor xenografts, canine, and human tumors [53]. Heating at $40^{\circ} \mathrm{C}$ potentiated the cytotoxicity of CT drugs in human maxillary carcinoma cells [28], and the cytotoxicity was further increased on heating to $43.5-44^{\circ} \mathrm{C}$ [54]. In contrast, another preclinical study showed no such dependency at $41-43.5^{\circ} \mathrm{C}$ [55]. An in vitro study showed that apoptosis in human keratinocytes occurred at temperatures of $39^{\circ} \mathrm{C}$ and above [56]. However, the majority of studies show synergistic actions of HT with RT and CT at temperatures above $41^{\circ} \mathrm{C}[5,57]$, leading to the inhibition of DNA repair and chromosomal aberrations, induction of DNA breaks by RT and CT, and protein damage as an underlying molecular event of heat treatment $[5,58,59]$. To benefit from additive and synergistic effects of HT when combined with RT and/or CT, uniform temperature in the target volume should be delivered during the whole treatment course.

The temperature metrics are used to present the heating temperature achieved during treatment, not only in the target volume, which encompasses the tumor, but also for adjacent healthy tissue. $T_{90}, T_{80}, T_{50}, T_{20}$, and $T_{10}$ are considered to be less sensitive than $\mathrm{T}_{\min }, \mathrm{T}_{\mathrm{avg}}$ and $\mathrm{T}_{\max }$, due to the number and arbitrary positioning of sensors in the tissue. 
Such temperature metrics can be used to understand the response to heat of various cancer types for a specific duration and, at the same time, the heat-induced effects on surrounding normal tissues. However, except for $\mathrm{T}_{\min }$ and $\mathrm{T}_{\max }$, most descriptive metrics of temperature have no specific reference values yet (Table 2).

Table 2. Reference temperature metrics.

\begin{tabular}{cc}
\hline Temperature Metrics & Reference Value $\left({ }^{\circ} \mathbf{C}\right)$ \\
\hline $\mathrm{T}_{\min }$ & 39 \\
$\mathrm{~T}_{\max }$ & 44 \\
$\mathrm{~T}_{\mathrm{avg}}$ & Undefined \\
$\mathrm{T}_{10}$ & Undefined \\
$\mathrm{T}_{20}$ & Undefined \\
$\mathrm{T}_{50}$ & $\geq 41^{*}$ \\
$\mathrm{~T}_{80}$ & Undefined \\
$\mathrm{T}_{90}$ & $\geq 40^{*}$ \\
\hline
\end{tabular}

* According to ESHO guidelines for superficial HT [43].

$\mathrm{T}_{50}$ and $\mathrm{T}_{90}$ reference values are defined according to ESHO guidelines for treatment with superficial HT, but not for the deep HT technique. No reference values for temperature metrics are based on experimental data (Table 2), even though temperature distributions can be better controlled in preclinical than in clinical studies. In an in vivo study, no temperature variations were observed in tumors as they were recorded intratumorally [15]. Temperature at a reference value with minor variations $\left( \pm 0.05^{\circ} \mathrm{C}\right)$ was reported in a vitro study [60]. In contrast, the temperature data recorded in patients are limited for various reasons. For example, thermistor probes inserted in deep-seated tumors in patients have the potential to cause complications or sometimes are impractical to insert intraluminally or intratumorally [61]. The value of the lowest temperature achieved during HT treatment is shown to have a prognostic role in describing the biological effects of HT. According to an in vivo study, $\mathrm{T}_{90}$ was a predictive parameter of reoxygenation and radiosensitization effects [62]. An in vitro experiment which investigated the difference in thermal sensitivity between hypoxic and oxic cells demonstrated that direct cytotoxicity induced by HT is more selective to the hypoxic cells [7]. Thus, temperatures required to achieve comparable thermal enhancement effect of HT vary depending on tissue type and characteristics.

\subsection{Heating Duration}

Temperature fluctuations, such as a decrease by $0.5^{\circ} \mathrm{C}$, have been shown to have a strong effect on the extent of cell kill, which was compensated by doubling the heating duration $[6,63]$. Therapeutic ratio, defined as the ratio of thermosensitive liposomal doxorubicin delivered to the heated tumor increased from 1.9-fold with $10 \mathrm{~min}$ heating to 4.4-fold with $40 \mathrm{~min}$ heating [64]. In an in vivo study, TER for mouse mammary adenocarcinoma $(\mathrm{C} 3 \mathrm{H})$ increased with respect to heating exposure longer than $30 \mathrm{~min}$ at $41.5^{\circ} \mathrm{C}$ [15]. A study used mouse leukemia, human cervical carcinoma (HeLa), and Chinese hamster ovary ( $\mathrm{CHO}$ ) cells to demonstrate that the time required to kill $90 \%$ of the cells at $43^{\circ} \mathrm{C}$ varied according to type [65]. The survival data from different tissues were analyzed using the Arrhenius equation to understand the effect of $t_{\text {treat }}$ for different cell types [66]. These analyses showed that the reference $t_{\text {treat }}$ value is set at $60 \mathrm{~min}$ when heating constantly at reference temperature (Table 3).

Table 3. Reference heating duration parameters for HT.

\begin{tabular}{cc}
\hline Heating Duration Parameters & Reference Value $(\mathrm{min})$ \\
\hline$t_{\text {pre }}$ & undefined \\
$t_{\text {treat }}$ & $60^{1}$ \\
\hline
\end{tabular}

${ }^{1}$ According to the Arrhenius plot [66]. 
Heating for longer than $60 \mathrm{~min}$ is restricted by thermotolerance, which was observed after 20 min while heating at $43.5^{\circ} \mathrm{C}$ [67]. In addition, the surviving fraction of asynchronous $\mathrm{CHO}$ cells heated to $41.5^{\circ} \mathrm{C}$ was decreased with increasing $t_{\text {treat }}$, until the thermotolerance effect appeared [21]. Thermotolerance is activated by different forms of stress including heat exposure for a specific time [68], which depends on the temperature and the amount of HT damage induced [69]. In an experimental study, the effect of thermotolerance was observed using the human tumor cell line (HTB-66) and CHO cells after $4 \mathrm{~h}$ of heating at $42.5{ }^{\circ} \mathrm{C}$ and $3 \mathrm{~h}$ of heating at either 42.5 or $43{ }^{\circ} \mathrm{C}$ [70]. The degree of thermotolerance is determined by cell type, heating temperature, and time of heating including the interval between successive heat treatments [71].

\subsection{Thermal Dose}

The relationship between temperature and $t_{\text {treat }}$ was demonstrated experimentally in two preclinical studies, which showed that the same thermal enhancement of ionizing radiation in cells lines was achieved by heating for $7-11 \mathrm{~min}$ at $45^{\circ} \mathrm{C}$ or for $120 \mathrm{~min}$ at $42{ }^{\circ} \mathrm{C}[26,72]$. It was also shown that different survival rates were obtained when heating asynchronous $\mathrm{CHO}$ cells to different temperatures for varying $t_{\text {treat }}$ [66]. These preclinical data showed that heating temperature and $t_{\text {treat }}$ influence thermal damage. The relationship of temperature and $t_{\text {treat }}$ to the biological effects induced by HT is described using the Arrhenius equation, which models the relationship of the inactivation rate in a biological system [21]. This led to the discovery that the relationship between temperature and $t_{\text {treat }}$ depends on the activation energy required to induce a particular HT-induced biological event, such as protein denaturation $[59,66]$. The thermal dose concept, $\mathrm{CEM} 43^{\circ} \mathrm{C}$, was established to account for the biological effects induced by HT in terms of both temperature and $t_{\text {treat }}$ [21]. More specifically, $\mathrm{CEM} 43^{\circ} \mathrm{C}$ calculates the equivalent time of a HT treatment session by correlating temperature, $t_{\text {treat }}$ and inactivation rate of a biological effect induced by heat based on the Arrhenius equation. The reference temperature of $43^{\circ} \mathrm{C}$ was shown as a breakpoint in the Arrhenius plot with a steeper slope between 41.5 and $43{ }^{\circ} \mathrm{C}$ in comparison to $43-57^{\circ} \mathrm{C}$ [66]. The threshold values of $\mathrm{CEM} 43^{\circ} \mathrm{C}$ for tissue damage differ for specific tissues as identified in in vivo studies and are reviewed elsewhere $[70,73,74]$. In addition, these data underline that $\mathrm{CEM} 43^{\circ} \mathrm{C}$ is an important parameter that has biological validity to assess the thermal damage in tissues. $\mathrm{CEM} 43^{\circ} \mathrm{CT}_{90}$ is one of the most frequently used thermal dose descriptors at $\mathrm{T}_{90}$, not only in clinical, but also in experimental settings. In an in vivo study, Thrall et al. [75] showed a relationship between $\mathrm{CEM}_{4}{ }^{\circ} \mathrm{CT}_{90}$ and local control in canine sarcomas, but not with $\mathrm{CEM}_{4} 3^{\circ} \mathrm{CT}_{50}$ and $\mathrm{CEM}_{4}{ }^{\circ} \mathrm{CT}_{10}$. Another in vivo study using breast (MDA-MB-231) and pancreatic cancer (BxPC-3) xenografts showed that at relatively low values of $\mathrm{CEM} 43^{\circ} \mathrm{CT}_{90}$, tumor volumes could be reduced by exposure to heat alone [76]. However, none of the preclinical studies proposed reference values for clinical validation, as shown in Table 4.

Table 4. Reference thermal dose parameters for HT.

\begin{tabular}{cc}
\hline Thermal Dose Parameters & Reference Value \\
\hline $\mathrm{CEM}^{\circ} 3^{\circ} \mathrm{CT}_{10}$ & Undefined (min) \\
$\mathrm{CEM}^{\circ} 3^{\circ} \mathrm{CT}_{50}$ & Undefined (min) \\
$\mathrm{CEM}^{\circ} 3^{\circ} \mathrm{CT}_{90}$ & Undefined $(\mathrm{min})$ \\
TRISE & Undefined $\left({ }^{\circ} \mathrm{C}\right)$ \\
AUC & Undefined $\left({ }^{\circ} \mathrm{C}-\mathrm{min}\right)$ \\
\hline
\end{tabular}

Although there is no reference threshold value for the $\mathrm{CEM} 43^{\circ} \mathrm{C}$, its efficacy to predict tumor response and local control has been experimentally proven $[75,77]$. $\mathrm{CEM} 43^{\circ} \mathrm{C}$ is considered as a thermal dose parameter with few weaknesses which have been discussed elsewhere [78]. 


\subsection{Number of HT Sessions}

Thermotolerance is an undesirable side effect of HT which renders tumor cells insensitive to heat treatment for 48 to $72 \mathrm{~h}$ [79]. Thermotolerance consists of an induction phase, a development phase, and a decay phase. Each of these components may have its own temperature dependence as well as dependence on other factors, such as $\mathrm{pH}$ and presence of nutrients [80]. Thermotolerance plays an important role on how HT sessions are scheduled during the treatment course. An in vivo study using $\mathrm{C} 3 \mathrm{H}$ mouse mammary carcinoma confirmed that preheating for $30 \mathrm{~min}$ at $43.5^{\circ} \mathrm{C}$ induced thermotolerance for the next heating session [81]. Twice weekly heating to $43^{\circ} \mathrm{C}$ for $60 \mathrm{~min}$ in combination with RT at 3 Gray (Gy) per fraction for 4 weeks was shown to result in a steady state decline in oxygenation level suggesting vascular thermotolerance [82]. In comparison, Nah et al. reported that heating at $42.5^{\circ} \mathrm{C}$ for $60 \mathrm{~min}$ could render the tumor blood vessels resistant to the next heating session after an interval of $72 \mathrm{~h} \mathrm{[83].} \mathrm{It} \mathrm{has} \mathrm{also} \mathrm{been} \mathrm{shown} \mathrm{that}$ when HT was delivered daily with RT 5 days a week, no significant thermal enhancement could be detected in comparison to one single HT session, even when heat was delivered simultaneously or sequentially [84]. With the agreement of these findings, $\mathrm{N}_{\text {week }}$ is defined as 1 or 2 sessions separated by at least $72 \mathrm{~h}$ (Table 5 ).

Table 5. Reference HT treatment session parameters. N: positive constant value.

\begin{tabular}{cc}
\hline Heating Session Parameter & Reference Value $(N)$ \\
\hline $\mathrm{N}_{\text {total }}$ & Defined $^{1}$ \\
$\mathrm{~N}_{\text {week }}$ & $1-2^{2}$ \\
\hline
\end{tabular}

${ }^{1}$ Depending on RT and CT schedules; ${ }^{2}$ Depending on cancer site.

In summary, HT should be delivered once or twice weekly, taking into account the type of cancer, RT fractionation and CT drug scheduling. Due to logistical reasons, the $\mathrm{N}_{\text {total }}$ usually depends on, the treatment plan for different cancer sites, number of RT fractions or number of CT cycles (Table 5).

\subsection{Time Interval Parameter between $H T$ and $R T$ and/or $C T$}

The $t_{\text {int }}$ between HT and RT and/or CT treatment is another parameter that affects sensitization due to time-dependent biological effects and its contribution to thermotolerance.

Recently, an in vitro study of human papillomavirus (HPV)-positive (HPV16 ${ }^{+}, \mathrm{HPV} 18+$ ) and HPV-negative cell lines that were treated with HT either 0,2 and $4 \mathrm{~h}$ before and after RT showed that the shortest $t_{i n t}$ resulted in lower cell survival fractions and decreased DNA damage repair [85]. The influence of $t_{\text {int }}$ has been investigated in an in vivo study, which reported that TER is greatest when heat and radiation are delivered simultaneously [15]. Unfortunately, simultaneous delivery is currently technically impossible in clinical routine and therefore heat and radiation are usually delivered sequentially. A very short $t_{\text {int }}$ of approximately five min is considered as an almost simultaneous application [86]. Dewey et al. concluded that HT should be applied simultaneously or within 5-10 min either side of radiation to benefit maximally from the radiosensitizating effect of heat [6]. TER is decreased faster for the normal cells than for cancerous cells when $\mathrm{t}_{\text {int }} \leq 4 \mathrm{~h}$ between HT and RT [15]. Thus, it can be argued that a slightly longer $t_{\text {int }}$ could ensure the sparing of normal tissue from radiosensitization before or after $\mathrm{RT}$. A $t_{\mathrm{int}}$ longer than $4 \mathrm{~h}$, no sensitization effects induced by HT were observed $[15,85]$. The wide range of acceptable $t_{\text {int }}$ values reported in experimental studies is from 0 (when CT is delivered during HT) to $4 \mathrm{~h}$ (Table 6).

Table 6. Reference $t_{\text {int }}$ parameter for HT in combination with RT or CT.

\begin{tabular}{cc}
\hline Time Interval Parameter & Reference Value (min) \\
\hline $\mathrm{t}_{\text {int }}$ & $0-240$ \\
\hline
\end{tabular}


In contrast to RT, CT can be given simultaneously or immediately after or before HT [87]. A preclinical study, in which cisplatin and heat were used to treat $\mathrm{C} 3 \mathrm{H}$ xenografts, showed that a higher additive effect can be obtained when cisplatin was given 15 min before HT in comparison with an interval longer than $4 \mathrm{~h}$ [55].

Furthermore, HT has been shown to sensitize the effects of gemcitabine at $43{ }^{\circ} \mathrm{C}$ when the drug was given $24 \mathrm{~h}$ after heating [88], whereas another study showed an optimal effect when the drug was given 24-48 h before heating [89]. The type of CT agent and its interaction with heat are factors which determine the $t_{\text {int }}$ between HT and CT (Table 6).

\subsection{Sequencing of HT in Combination with and RT and/or CT}

An additional predictive parameter for the effectiveness of radiosensitization and chemosensitization is the sequencing of heat prior to or after the application of RT or CT. Usually, HT and RT are delivered sequentially but there is no consensus as to the optimal sequence. An in vivo study by Overgaard investigated the impact of sequence and interval between the two modalities on local tumor control and normal tissue damage in a murine breast cancer model and found that the sequence did not have any significant effect on the thermal enhancement in tumor tissues [15]. However, an experimental study using Chinese hamster ovary (HA-1) and mouse mammary sarcoma (EMT-6) cell lines showed that sequencing of radiation and heat altered radiosensitivity for these two cancer cell types [90]. HT before RT showed more thermal enhancement in synchronous HA-1 cell lines and the opposite sequence increased the thermal enhancement in EMT-6 cell lines. Other experimental studies reported no impact of the sequence of RT and HT in V79 cells on thermal enhancement [26,72]. In line with these results, an experimental study with HPV cell lines showed no difference in radiosensitization or cell death when heat was delivered prior or after radiation [85]. Due to conflicting results with regard to the treatment sequencing of HT and RT, additional preclinical mechanistic studies on different cell types are required.

An in vivo study where heat was combined with cisplatin CT showed that simultaneous application of both treatments resulted in prolonged tumor growth delay in comparison with administration of cisplatin after HT [55]. Another study found that simultaneous exposure of human colorectal cancer (HCT116) cells to HT and doxorubicin was more effective than sequential administration because of higher intercellular drug concentrations at $42{ }^{\circ} \mathrm{C}$ [91]. In conclusion, better insight into the interaction of various CT drugs with HT and RT is required to define the optimal sequencing of specific drugs and RT dose.

\section{Evidence for the Predictive Values of Thermometric Parameters in Clinical Studies Combining HT with RT}

Numerous prospective and retrospective clinical studies have been conducted to assess the efficacy of HT in combination with RT for treating superficial and deep-seated tumors. The design of most clinical studies was based on the translation of experimental findings aiming to reproduce benefit of HT when combined with RT.

Tables 7 and 8 show the results of the most important clinical studies. The prospective clinical studies in Table 7 reported improved clinical results, apart from the study by Mitsumori et al. which did not show a significant difference in the primary clinical endpoint of local tumor control between two treatment arms [92]. The underlying reason could have been differences in RT dose prescriptions and missing patient treatment data. Although the study showed a significant difference in progression free survival, this was judged to be not a substantial benefit. The authors stressed the need for internationally standardized treatment protocols for the combination of HT and RT.

In reality, temperature and thermal dose are usually reported as post-treatment data recordings (Tables 2 and 4) to account for temperature homogeneity or sensitivity. Even though temperature cannot always be measured invasively, depending on the location of the tumor, a strong correlation was reported between intratumoral and intraluminal temperatures, suggesting that intraluminal temperature measurements are a good surrogate for 
pelvic tumor measurements [50,93]. In addition, retrospective studies showed that a higher intra-esophageal temperature $\left(>41^{\circ} \mathrm{C}\right)$ predicts longer overall survival, improved local control and metastasis-free rate [94,95]. The difficulty of performing invasive measurements was illustrated by a randomized phase III study by Chi et al. [96] in which only 3 out of 29 patients with bone metastases had directly measured intratumoral temperature. In the study by Nishimura et al. [97], the HT session was defined as effective if an intratumoral temperature exceeded $42{ }^{\circ} \mathrm{C}$ for more than $20 \mathrm{~min}$. However, according to the Arrhenius relationship, this is not considered long enough to induce a significant biological effect [21].

Another obstacle during HT is the non-standardized methodology for describing the temporal and spatial variance of temperature fields. Several groups have investigated the correlation between various temperature metrics. The study by Oleson et al. showed that $\mathrm{T}_{\min }$, tumor volume, radiation dose, and heating technique play significant roles in predicting treatment response for patients treated with RT in combination with HT [29]. In contrast, Leopold et al. reported that the more robust parameters $T_{90}, T_{50}$, and $T_{10}$ are better temperature descriptors and predictors of histopathologic outcome than $\mathrm{T}_{\min }$ and $\mathrm{T}_{\max }$ [32]. The median $\mathrm{T}_{\min }, \mathrm{T}_{\min }$ during the first heat treatment and tumor volume were reported to be factors predictive for the duration of cancer response (Table 7) [98], even though it is considered that skin surface temperature is not representative for superficial tumors and cannot be associated with clinical outcomes [42]. For deep-seated tumors, Tilly et al. reported that $\mathrm{T}_{\max }$ was a predictive treatment parameter for prostate-specific antigen (PSA) control [99]. The relationship of high $\left(\mathrm{T}_{\mathrm{avg}} \geq 41.5^{\circ} \mathrm{C}\right)$ and low $\left(\mathrm{T}_{\mathrm{avg}}<41.5^{\circ} \mathrm{C}\right)$ tumor temperature with clinical response has been analyzed in a study by Masunaga et al. [100]. They showed that heating the tumor to temperatures of $\mathrm{T}_{\mathrm{avg}} \geq 41.5^{\circ} \mathrm{C}$ for a duration of 15-40 min achieved better tumor down-staging and better tumor degeneration rates [100]. This finding supports the concept that direct cytotoxic effects of HT are enhanced at temperatures higher than $41^{\circ} \mathrm{C}$, as suggested in preclinical studies [5,57]. A higher response rate was also reported when tumors were heated with $\mathrm{T}_{\mathrm{avg}}>42{ }^{\circ} \mathrm{C}$ for $3-5 \mathrm{HT}$ sessions [97]. In contrast, a study showed no difference in clinical outcome when patients were treated with mean $\mathrm{T}_{\min }=40.2^{\circ} \mathrm{C}, \mathrm{T}_{\max }=44.8^{\circ} \mathrm{C}$ or $\mathrm{T}_{\mathrm{avg}}=42.5^{\circ} \mathrm{C}$ for $\mathrm{N}_{\text {total }}$ of 2 or 6 [24]. Other studies also reported no impact of $\mathrm{N}_{\text {total }}$ and $\mathrm{N}_{\text {week }}$ on clinical outcome $[40,101]$. The contradictory results derived from clinical studies with regard to the predictive power of temperature descriptors and $\mathrm{N}_{\text {total }}$ are why we did not list reference values for these descriptors in Table 5.

The predictive role of thermal dose has been investigated in both prospective and retrospective clinical studies (Tables 7 and 8). However, there is still no conclusion about the values for thermal dose that should be obtained during HT treatment for maximal enhancement effect. In prospective studies (Table 7), the correlation between thermal dose and treatment outcome is rarely reported. Retrospective studies reported that thermal dose, $\mathrm{CEM} 43^{\circ} \mathrm{C}$, is an adequate predictor of treatment response and its best prognostic descriptor is $\mathrm{CEM}_{43}{ }^{\circ} \mathrm{CT}_{90}[32,33,36-38,102]$. 
Table 7. Prospective clinical studies using RT in combination with HT.

\begin{tabular}{|c|c|c|c|c|c|c|c|c|c|}
\hline Author(s) & Cancer Site, $n$ & $\begin{array}{l}\text { RT Dose (Gy) } \\
\text { /Fractions }\end{array}$ & $\begin{array}{l}\text { Temperature } \\
\text { Metrics }\left({ }^{\circ} \mathrm{C}\right)\end{array}$ & $\begin{array}{c}\text { HT } \\
\text { Session }\end{array}$ & $\begin{array}{c}\mathbf{t}_{\text {treat }} \\
\text { (min) }\end{array}$ & $\begin{array}{c}\text { Thermal } \\
\text { Dose (min) }\end{array}$ & $\begin{array}{c}\mathbf{t}_{\text {int }} \\
(\mathbf{m i n})\end{array}$ & Sequence & $\begin{array}{c}\text { Clinical Outcome } \\
\text { (Comment) }\end{array}$ \\
\hline $\begin{array}{c}\text { Chi } \\
\text { et al. [96] }\end{array}$ & $\begin{array}{l}\text { Bone metastases, } \\
\quad n=29\end{array}$ & $30.0 / 10$ & $\begin{array}{c}\mathrm{T}_{\max }{ }^{\dagger}: \\
41.9 \pm 1.2\end{array}$ & $\begin{array}{l}\mathrm{N}_{\text {total }}: \\
4 \\
\mathrm{~N}_{\text {week }}: \\
\quad 2\end{array}$ & 40 & n.r. & 120 & HT after RT & $\begin{array}{l}\text { - } \quad \text { Increased 3-months } \\
\text { radiologic CR }{ }^{1} \text { and } \mathrm{PR}^{2} \\
\text { rate: } 37.9 \%(11 / 29) \text { and } \\
66.7 \%(10 / 15) \text {, respectively. } \\
\text { No grade III toxicity was } \\
\text { reported. } \\
\text { HT increased pain control } \\
\text { rate, no progression of pain } \\
\text { achieved after } 29 \text { days. } \\
\text { (correlation of thermometric } \\
\text { parameters with clinical outcome } \\
\text { not presented) }\end{array}$ \\
\hline Valdagni et al. [103] & $\begin{array}{c}\text { Head \& neck, } \\
\quad n=18\end{array}$ & $\begin{array}{l}64.0-70.0 \\
/ 32-35\end{array}$ & $\begin{array}{c}\mathrm{T}_{\max }{ }^{\dagger}: \\
43.3 \pm 0.2 \\
\mathrm{~T}_{\min }^{+}: \\
40.4 \pm 0.2 \\
\mathrm{~T}_{50}: \\
41.8 \pm 0.2 \\
\mathrm{~T}_{90}: \\
39.8 \pm 0.02\end{array}$ & $\begin{array}{c}\mathrm{N}_{\text {total }}: \\
6 \\
\mathrm{~N}_{\text {week }}: \\
2\end{array}$ & n.r. & $\begin{array}{c}\max \\
\mathrm{CEM}_{2} .5^{\circ} \mathrm{C}^{5} \text { : } \\
83.84 \pm 9.4 \min \\
\mathrm{CEM} 42.5^{\circ} \mathrm{C}: \\
12.8-2.1\end{array}$ & $20-25$ & HT after RT & $\begin{array}{ll}\text { - } & \text { 3-month CR: } 83.3 \%(15 / 18), \\
& \text { PR: } 5.56 \%(1 / 18), \mathrm{PD}^{3} \text { rate } \\
\text { of } 11.1 \%(2 / 18), \text { overall } \\
\text { improved LC } 4 \text {. } \\
\text { - 5-year nodal control rate: } \\
\text { 68.6\% with TER: } 2.83 \text {. } \\
\text { - } \mathrm{N}_{\text {total }} \text { of two or six yielded } \\
\text { similar results }(80 \% \text { CR } \\
\text { with } 6 \text { sessions vs. } 87 \% \text {, } \\
\text { with } 2 \text { sessions). } \\
\text { No enhanced acute or late } \\
\text { toxicities were reported. } \\
\text { Extensive thermal analysis } \\
\text { performed: no relation } \\
\text { between thermometric } \\
\text { parameters and response } \\
\text { or toxicity. }\end{array}$ \\
\hline
\end{tabular}


Table 7. Cont.

\begin{tabular}{|c|c|c|c|c|c|c|c|c|c|}
\hline Author(s) & Cancer Site, $n$ & $\begin{array}{l}\text { RT Dose (Gy) } \\
\text { /Fractions }\end{array}$ & $\begin{array}{l}\text { Temperature } \\
\text { Metrics }\left({ }^{\circ} \mathrm{C}\right)\end{array}$ & $\begin{array}{c}\text { HT } \\
\text { Session }\end{array}$ & $\begin{array}{c}t_{\text {treat }} \\
\text { (min) }\end{array}$ & $\begin{array}{c}\text { Thermal } \\
\text { Dose (min) }\end{array}$ & $\begin{array}{c}t_{\text {int }} \\
\text { min) }\end{array}$ & Sequence & $\begin{array}{c}\text { Clinical Outcome } \\
\text { (Comment) }\end{array}$ \\
\hline Jones et al. [35] & $\begin{array}{c}\text { Superficial } \\
\text { cancers, } n=56\end{array}$ & $\begin{array}{c}30.0-66.0 \\
/ 15-33 \\
\text { when } \\
\text { previously } \\
\text { unirradiated } \\
60.0-70.0 \\
/ 30-35\end{array}$ & n.r. & $\begin{array}{c}\mathrm{N}_{\text {total }}: \\
4-10 \\
\mathrm{~N}_{\text {week }}: \\
\quad 2\end{array}$ & $60 \mathrm{~min}$ & $\begin{array}{c}\mathrm{CEM}_{4} 3^{\circ} \mathrm{CT}_{90}{ }^{\dagger}: \\
(0.57-36.21)\end{array}$ & n.r. & n.r. & $\begin{array}{ll}\text { - } & \text { CR: } 66.1 \%, \mathrm{LC} \text { for } \\
\text { pre-irradiated tumors: } 48 \% \text {. } & \text { CEM43 }{ }^{\circ} \mathrm{CT}_{90} \text { associated } \\
\text { - } & \text { with CR rate. } \\
\text { - Greater than } \\
10 \text { CEM } 43^{\circ} \mathrm{CT} 90 \text { showed a } \\
\text { significant LC benefit. } \\
\text { The improvement in LC } \\
\text { was most pronounced for } \\
\text { patients who were } \\
\text { previously irradiated. } \\
\text { No significant toxicity or } \\
\text { survival benefit } \\
\text { was reported. }\end{array}$ \\
\hline $\begin{array}{l}\text { van der Zee } \\
\text { et al. [104] }\end{array}$ & $\begin{array}{l}\text { Locally advanced } \\
\text { pelvic tumors, } \\
n=182\end{array}$ & $\begin{array}{c}\text { Bladder: } \\
\text { 66.0-70.0 } \\
\text { /33-35 } \\
\text { Cervix: } \\
40.0-50.0 \\
\text { /23-28 } \\
\text { with } \\
\text { HDR-IRT } 23 \\
\text { ('192 Ir): } \\
14.0 \\
\text { or LDR-IRT } 24 \\
\text { (192 Ir): } \\
\text { 20.0-30.0 } \\
\text { Rectum: } \\
46.0-50.0 \\
\text { /20-22 }\end{array}$ & n.r. & $\begin{array}{c}\mathrm{N}_{\text {total }}: \\
5 \\
\mathrm{~N}_{\text {week }}: \\
1\end{array}$ & 60 & n.r. & $60-240$ & HT after RT & $\begin{array}{l}\text { - } \quad \text { CR for all patients: } 55 \% \text {, } \\
\text { bladder: } 73 \% \text {, cervical: } \\
83 \% \text {, rectal: } 21 \% \text {. } \\
\text { - } 3 \text {-year LC for all patients: } \\
38 \% \text {, for bladder: } 42 \% \text {, for } \\
\text { cervical: } 61 \% \text {, for rectal: } \\
16 \% \text {. } \\
\text { 3-year OS }{ }^{6} \text { rate for all } \\
\text { patients: } 30 \% \text {, for bladder: } \\
28 \% \text {, for cervical: } 51 \% \text {, for } \\
\text { rectal: } 22 \% \text {. } \\
\text { 2.2\% had grade III-IV } \\
\text { HT-related toxicity. } \\
\text { (correlation of thermometric } \\
\text { parameters with clinical outcome } \\
\text { not presented) }\end{array}$ \\
\hline
\end{tabular}


Table 7. Cont.

\begin{tabular}{|c|c|c|c|c|c|c|c|c|c|}
\hline Author(s) & Cancer Site, $n$ & $\begin{array}{l}\text { RT Dose (Gy) } \\
\text { /Fractions }\end{array}$ & $\begin{array}{l}\text { Temperature } \\
\text { Metrics }\left({ }^{\circ} \mathrm{C}\right)\end{array}$ & $\begin{array}{c}\text { HT } \\
\text { Session }\end{array}$ & $\begin{array}{c}t_{\text {treat }} \\
\text { (min) }\end{array}$ & $\begin{array}{c}\text { Thermal } \\
\text { Dose (min) }\end{array}$ & $\begin{array}{c}t_{\text {int }} \\
(\min )\end{array}$ & Sequence & $\begin{array}{c}\text { Clinical Outcome } \\
\text { (Comment) }\end{array}$ \\
\hline Harima et al. [105] & $\begin{array}{c}\text { Cervix cancer, } \\
n=20\end{array}$ & $\begin{array}{c}52.2 / 29 \\
\text { with } \\
\text { HDR-IRT } \\
\text { (192 Ir): } \\
30.0 / 4\end{array}$ & $\begin{array}{c}\mathrm{T}_{\max }{ }^{+}: \\
41.8 \pm 1.1 \\
\mathrm{~T}_{\text {avg }}{ }^{+}: \\
40.6 \pm 1.0 \\
\mathrm{~T}_{\min }{ }^{+}: \\
39.6 \pm 0.9\end{array}$ & $\begin{array}{c}\mathrm{N}_{\text {total }}: \\
3 \\
\mathrm{~N}_{\text {week }}: \\
1\end{array}$ & 60 & n.r. & 30 & HT after RT & 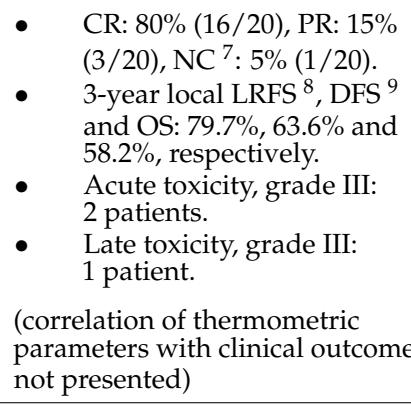 \\
\hline Mitsumori et al. [92] & $\begin{array}{l}\text { Locally advanced } \\
\text { non-small cell lung } \\
\text { cancers, } n=40\end{array}$ & $\begin{array}{c}66.0-70.0 \\
/ 33-38\end{array}$ & $\begin{array}{c}\mathrm{T}_{\max }{ }^{+}: 41.3 \\
(37.7-44.0) \\
\mathrm{T}_{\min }{ }^{+}: 39.5 \\
(35.5-41.7) \\
\mathrm{T}_{\text {avg }}{ }^{+}: 40.3 \\
(37.0-42.7)\end{array}$ & $\begin{array}{c}\mathrm{N}_{\text {total }}: \\
7 \\
\mathrm{~N}_{\text {week }}: \\
1\end{array}$ & 60 & n.r. & n.r. & n.r. & $\begin{array}{ll}\text { - } & \mathrm{RR}^{10}: 45.0 \% . \\
& \text { 1-year LRFS and OS: } 67.5 \% \\
& \text { and } 43 \% \text {, respectively. } \\
\text { - } & \text { Acute toxicity, grade II: } 4 \\
& \text { patients and grade III: } 2 \\
& \text { patients. } \\
\text { - } & \text { Late toxicity, grade II: } 3 \\
& \text { patients and no grade III. } \\
\text { (correlation of thermometric } \\
\text { parameters with clinical outcome } \\
\text { not presented) }\end{array}$ \\
\hline Masunaga et al. [100] & $\begin{array}{l}\text { Urinary bladder } \\
\text { cancer, } n=28\end{array}$ & $24.0 / 6$ & $\begin{array}{c}\mathrm{T}_{\mathrm{avg}}{ }^{\dagger}: \\
41.5 \pm 1.1 \\
(39-44)\end{array}$ & $\begin{array}{c}\mathrm{N}_{\text {total }}: \\
4 \\
\mathrm{~N}_{\text {week }}: \\
2\end{array}$ & $15-40$ & n.r. & n.r. & HT after RT & $\begin{array}{ll}\text { - } & \mathrm{T}_{\text {avg }} \geq 41.5^{\circ} \mathrm{C} \text { achieved } \\
\text { better results: } 83.3 \% \\
(10 / 12) \text { tumor } \\
\text { down-staging and } \\
\text { degeneration, } 0 \% \text { local } \\
\text { recurrence, } 33 \% \text { distant } \\
\text { metastasis, in contrast with } \\
\mathrm{T}_{\text {avg }}<41.5^{\circ} \mathrm{C} \text {. } \\
\text { Survival rate was higher if } \\
\mathrm{T}_{\text {avg }} \geq 41.5^{\circ} \mathrm{C} \text { than } \\
\mathrm{T}_{\text {avg }}<41.5^{\circ} \mathrm{C} \text {. } \\
\text { The toxicity associated } \\
\text { with HT: pain } \\
\text { during treatment. }\end{array}$ \\
\hline
\end{tabular}


Table 7. Cont.

\begin{tabular}{|c|c|c|c|c|c|c|c|c|c|}
\hline Author(s) & Cancer Site, $n$ & $\begin{array}{l}\text { RT Dose (Gy) } \\
\text { /Fractions }\end{array}$ & $\begin{array}{l}\text { Temperature } \\
\text { Metrics }\left({ }^{\circ} \mathrm{C}\right)\end{array}$ & $\begin{array}{c}\text { HT } \\
\text { Session }\end{array}$ & $\begin{array}{c}t_{\text {treat }} \\
\text { (min) }\end{array}$ & $\begin{array}{c}\text { Thermal } \\
\text { Dose (min) }\end{array}$ & $\begin{array}{c}t_{\text {int }} \\
\text { min) }\end{array}$ & Sequence & $\begin{array}{c}\text { Clinical Outcome } \\
\text { (Comment) }\end{array}$ \\
\hline Berdov et al. [106] & $\begin{array}{l}\text { Advanced rectal } \\
\text { cancer, } n=56\end{array}$ & $40.0 / 10$ & n.r. & $\begin{array}{l}\mathrm{N}_{\text {total }}: \\
4-5 \\
\mathrm{~N}_{\text {week }}: \\
\text { n.r. }\end{array}$ & 60 & n.r. & 10 & HT before RT & $\begin{array}{l}\text { 1-,2-,3-,4-, and 5-year } \\
\text { survival: } 61.8 \pm 6.6 \%, \\
48.1 \pm 6.7 \%, 43.9 \pm 6.7 \% \text {, } \\
35.6 \pm 6.4 \%, \text { and } \\
35.6 \pm 6.4 \% \text {. } \\
\text { The mean for CR rate } \\
(>50 \%): 53.6 \%(30 / 56) \text { and } \\
\text { for CR rate }(<50 \%): 23.3 \% \\
(13 / 56) .\end{array}$ \\
\hline Maluta et al. [107] & $\begin{array}{l}\text { Locally advanced high } \\
\text { risk prostate cancer, } \\
n=144\end{array}$ & $\begin{array}{c}70.0-76.0 \\
/ 35-38\end{array}$ & $\begin{array}{c}\text { Rectum } \\
\mathrm{T}_{\max }^{+}: \\
42.7 \\
\mathrm{~T}_{90}^{+}: \\
40.2 \\
(38.4-42.0) \\
\text { Bladder } \\
\mathrm{T}_{90}^{+}: \\
41.3 \\
(39.5-42.3)\end{array}$ & $\begin{array}{c}\mathrm{N}_{\text {total }}: \\
4 \\
\mathrm{~N}_{\text {week }}: \\
1\end{array}$ & n.r. & $\begin{array}{c}\mathrm{CEM} 40{ }^{\circ} \mathrm{CT}_{90}{ }^{\dagger}: \\
24.4 \\
(14.4-34.4)\end{array}$ & $15-30$ & HT before RT & $\begin{array}{l}\text { - } \quad \text {-year OS: } 87 \% \text { and 5-year } \\
\text { biochemical } \\
\text { progression-free survival: } \\
49 \% \text {. } \\
\text { No late grade III toxicity or } \\
\text { significant acute } \\
\text { HT-correlated toxicity. } \\
\text { (correlation of thermometric } \\
\text { parameters with clinical outcome } \\
\text { not presented) }\end{array}$ \\
\hline Leopold et al. [40] & $\begin{array}{c}\text { Superficial } \\
\text { cancers, } n=111\end{array}$ & $\begin{array}{l}24.0-70.0 \\
\quad / 7-28\end{array}$ & n.r. & $\begin{array}{c}\mathrm{N}_{\text {total }} \\
+: 4.5(1-6) \\
\text { for } \mathrm{N}_{\text {week }}=1 \\
\text { and } \\
7(2-13) \\
\text { for } \mathrm{N}_{\text {week }}=2 \\
\mathrm{~N}_{\text {week }}: 1-2\end{array}$ & 60 & n.r. & $30-90$ & HT after RT & $\begin{array}{ll}\text { - } & \text { CR: } 46 \% \text {, PR: } 34 \% \text {, OS: } 80 \% \text {. } \\
\text { - } & \mathrm{T}_{90} \text { was significantly } \\
\text { related to CR. } \\
\text { - } \quad \text { Cumulative minutes of } \mathrm{T}_{90} \\
\geq 40{ }^{\circ} \mathrm{C} \text { and logarithm of } \\
\text { RT dose were predictive of } \\
\text { both CR and OS. } \\
\text { - } \mathrm{T}_{\text {min }}, \mathrm{N}_{\text {week, }} \text { and } \mathrm{N}_{\text {total }} \\
\text { were not significantly } \\
\text { related to either end points. } \\
\text { - Toxicity, grade IV: } 1 \text { patient } \\
\text { and grade III: } 7 \text { patients. }\end{array}$ \\
\hline
\end{tabular}


Table 7. Cont.

\begin{tabular}{|c|c|c|c|c|c|c|c|c|c|}
\hline Author(s) & Cancer Site, $n$ & $\begin{array}{l}\text { RT Dose (Gy) } \\
\text { /Fractions }\end{array}$ & $\begin{array}{l}\text { Temperature } \\
\text { Metrics }\left({ }^{\circ} \mathrm{C}\right)\end{array}$ & $\begin{array}{c}\text { HT } \\
\text { Session }\end{array}$ & $\begin{array}{l}t_{\text {treat }} \\
\text { (min) }\end{array}$ & $\begin{array}{c}\text { Thermal } \\
\text { Dose (min) }\end{array}$ & $\begin{array}{c}\mathrm{t}_{\text {int }} \\
(\mathrm{min})\end{array}$ & Sequence & $\begin{array}{c}\text { Clinical Outcome } \\
\text { (Comment) }\end{array}$ \\
\hline Nishimura et al. [97] & $\begin{array}{l}\text { Colorectal cancer, } \\
\quad n=33\end{array}$ & $\begin{array}{l}40.0-70.0 \\
/ 25-35\end{array}$ & 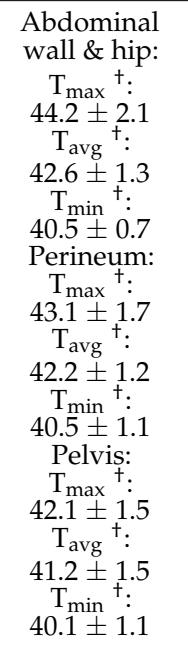 & $\begin{array}{c}\mathrm{N}_{\text {total }}: \\
2-14 \\
\mathrm{~N}_{\text {week }} \\
1-2\end{array}$ & $40-60$ & n.r. & $10-30$ & HT after RT & $\begin{array}{l}\text { 6- and 12-months LC: } 59 \% \\
\text { (17/29) and } 31 \%(8 / 21), \\
\text { respectively. } \\
\text { CR rate: } 11 \%(4 / 35) \text { and } \\
\text { PR: } 43 \%(15 / 35) \text {. } \\
\text { Better treatment response } \\
\text { of unresectable colorectal } \\
\text { cancers than recurrent } \\
\text { tumors. } \\
\text { Higher response rate of } \\
67 \% \text { reported when tumors } \\
\text { heated with } \mathrm{T}_{\text {avg }}{ }^{+}>42^{\circ} \mathrm{C} \\
\text { for } \mathrm{N}_{\text {total }}=3-5 \text {. } \\
\mathrm{N}_{\text {total }} \geq 5-14 \text { showed not } \\
\text { to increase the } \\
\text { response rate. }\end{array}$ \\
\hline
\end{tabular}


Table 7. Cont.

\begin{tabular}{|c|c|c|c|c|c|c|c|c|c|}
\hline Author(s) & Cancer Site, $n$ & $\begin{array}{l}\text { RT Dose (Gy) } \\
\text { /Fractions }\end{array}$ & $\begin{array}{l}\text { Temperature } \\
\text { Metrics }\left({ }^{\circ} \mathrm{C}\right)\end{array}$ & $\begin{array}{c}\text { HT } \\
\text { Session }\end{array}$ & $\begin{array}{c}t_{\text {treat }} \\
\text { (min) }\end{array}$ & $\begin{array}{c}\text { Thermal } \\
\text { Dose (min) }\end{array}$ & $\begin{array}{c}t_{\text {int }} \\
\text { (min) }\end{array}$ & Sequence & $\begin{array}{c}\text { Clinical Outcome } \\
\text { (Comment) }\end{array}$ \\
\hline Gabriele et al. [109] & $\begin{array}{l}\text { Inoperable or } \\
\text { recurrent parotid } \\
\text { carcinoma, } n=13\end{array}$ & $\begin{array}{l}\text { Inoperable: } \\
70.0 / 35 \\
\text { Recurrent: } \\
30.0 / 15\end{array}$ & $\begin{array}{c}\mathrm{T}_{\min }{ }^{+}: \\
40.28 \pm 0.83 \\
\mathrm{~T}_{\max }^{+}: \\
42.83 \pm 1.32\end{array}$ & $\begin{array}{c}\mathrm{N}_{\text {total }}: \\
4-10 \\
\mathrm{~N}_{\text {week }}: \\
\quad 2\end{array}$ & $30-45$ & n.r. & n.r. & n.r. & $\begin{array}{l}\text { CR: } 80 \%(16 / 20), \text { PR: } 20 \% \\
(4 / 20), \mathrm{LR} 11: 20 \%(16 / 20), \\
5 \text {-year actuarial LC: } \\
62.3 \pm 13.2 \% \text {. } \\
\text { Higher maximum } \\
\text { temperatures correlated } \\
\text { with acute toxicity and } \\
\text { maximum tumor diameter } \\
\text { but without statistical } \\
\text { significance. } \\
\text { Major acute toxicities } \\
\text { included three patients } \\
\text { (15\%) with superficial } \\
\text { necrosis, } 2 / 3 \text { healed } \\
\text { spontaneously within } 4 \text { to } \\
6 \text { months. } \\
\text { No correlation between } \\
\mathrm{T}_{\text {min }} \text { and } \mathrm{T}_{\text {max }} \text { and early or } \\
\text { long term response } \\
\text { was found. }\end{array}$ \\
\hline
\end{tabular}


Table 7. Cont.

\begin{tabular}{|c|c|c|c|c|c|c|c|c|c|}
\hline Author(s) & Cancer Site, $n$ & $\begin{array}{l}\text { RT Dose (Gy) } \\
\text { /Fractions }\end{array}$ & $\begin{array}{l}\text { Temperature } \\
\text { Metrics }\left({ }^{\circ} \mathrm{C}\right)\end{array}$ & $\begin{array}{c}\text { HT } \\
\text { Session }\end{array}$ & $\begin{array}{c}t_{\text {treat }} \\
\text { (min) }\end{array}$ & $\begin{array}{c}\text { Thermal } \\
\text { Dose (min) }\end{array}$ & $\underset{(\min )}{t_{\text {int }}}$ & Sequence & $\begin{array}{c}\text { Clinical Outcome } \\
\text { (Comment) }\end{array}$ \\
\hline Tilly et al. [99] & $\begin{array}{c}\text { Recurrent or } \\
\text { locally advanced } \\
\text { prostate cancer, } \\
n=22\end{array}$ & $68.4 / 38$ & $\begin{array}{c}\text { Primary } \\
\text { cancer: } \\
\mathrm{T}_{90}^{+}:: \\
40.7^{+} \pm 0.3 \\
\mathrm{~T}_{\max }^{+}: \\
41.4 \pm 0.4 \\
\text { Recurrent cancer: } \\
\mathrm{T}_{90}^{+}: \\
40.0^{+} \pm .8 \\
\mathrm{~T}_{\max }^{+}: \\
41.0^{+} \pm 0.7\end{array}$ & $\begin{array}{c}\mathrm{N}_{\text {total }}: \\
5-6 \\
\mathrm{~N}_{\text {week }}: \\
1\end{array}$ & $0-30$ & n.r. & 30 & $\begin{array}{c}\text { HT before RT } \\
\text { or } \\
\text { HT after RT }\end{array}$ & $\begin{array}{l}\text { 6-year OS: } 95 \% \text { and 6-year } \\
\text { - } \quad \text { Seve }{ }^{15}: 60 \% \text {. } \\
\text { toxicity: } 8 \text { patients and } \\
\text { grade II: } 2 \text { patients. } \\
\text { Late toxicity, grade III: } 1 \\
\text { patient and grade II: } \\
2 \text { patients. } \\
\text { No correlation between } \\
\text { thermal parameters and } \\
\text { toxicity. } \\
\text { The thermal parameters } \\
\text { were correlated with } \\
\text { clinical endpoints: toxicity, } \\
\text { PSA }{ }^{16} \text { control. } \\
\text { Tmax was the only relevant } \\
\text { predictive factor for } \\
\text { PSA control. }\end{array}$ \\
\hline Lutgens et al. [111] & $\begin{array}{l}\text { Locally advanced } \\
\text { cervical cancer, } \\
\qquad n=42\end{array}$ & $\begin{array}{c}50.0 / 25 \\
\text { with } \\
\text { HDR-IRT } \\
\text { (192 Ir): } \\
21.0 / 3 \\
\text { weekly } \\
\text { or LDR: } \\
\text { 32.0/1-2 } \\
\text { or MDR: } \\
29.0 / 1-2\end{array}$ & n.r. & $\begin{array}{c}\mathrm{N}_{\text {total }}: \\
5 \\
\mathrm{~N}_{\text {week }}: \\
1\end{array}$ & 60 & n.r. & $60-240$ & HT after RT & $\begin{array}{l}\text { - } \quad \text { Treatment failure in the } \\
\text { pelvis: } 19 \%(8 / 42) \text {. } \\
\text { - OS: comparable between } \\
\text { RT + CT and } \\
\quad \text { RT + HT groups. } \\
\text { - Toxicity of grade } \geq \text { III: } \\
\text { (correlation of thermometric } \\
\text { parameters with clinical outcome } \\
\text { not reported) }\end{array}$ \\
\hline Hurwitz et al. [112] & $\begin{array}{l}\text { Locally advanced } \\
\text { prostate cancer, } \\
\qquad n=37\end{array}$ & $\begin{array}{l}\text { 66.60-70.0 } \\
\text { /33-37 }\end{array}$ & $\begin{array}{c}\mathrm{T}_{\min }{ }^{+}: 40.1 \\
(37.5-41.8) \\
\mathrm{T}_{\max }{ }^{+}: 42.5 \\
(40.5-45.9) \\
\mathrm{T}_{\text {avg }}{ }^{+}: 41.2 \\
(39.2-42.8)\end{array}$ & $\begin{array}{c}\mathrm{N}_{\text {total }}: \\
2 \\
\mathrm{~N}_{\text {week }}: \\
1\end{array}$ & 60 & $\begin{array}{c}\mathrm{CEM} 43^{\circ} \mathrm{CT}_{90}{ }^{+}: \\
8.4\end{array}$ & 60 & HT before RT & $\begin{array}{l}\text { - } \quad \text {-year OS: } 94 \% \text { and failure } \\
\text { free: } 61 \% \text {. } \\
\text { 2-year DFS: } 84 \% \text { compared } \\
\text { with a rate of } 64 \% \text { for } \\
\text { similar patients on } 4 \text {-month } \\
\text { androgen suppression. } \\
\text { The difference in median } \\
\text { CEM43 }{ }^{\circ} \mathrm{CT}_{90} \text { between } \\
\text { these patient groups who } \\
\text { achieved } 2.8 \text { min and } \\
10.5 \text { min, respectively, was } \\
\text { significant }(p=0.004) \text {. } \\
\text { A small difference in DFS } \\
\text { in favor of patients treated } \\
\text { with higher temperatures. }\end{array}$ \\
\hline
\end{tabular}


Table 7. Cont.

\begin{tabular}{|c|c|c|c|c|c|c|c|c|c|}
\hline Author(s) & Cancer Site, $n$ & $\begin{array}{l}\text { RT Dose (Gy) } \\
\text { /Fractions }\end{array}$ & $\begin{array}{l}\text { Temperature } \\
\text { Metrics }\left({ }^{\circ} \mathrm{C}\right)\end{array}$ & $\begin{array}{l}\text { HT } \\
\text { Session }\end{array}$ & $\begin{array}{l}t_{\text {treat }} \\
\text { (min) }\end{array}$ & $\begin{array}{c}\text { Thermal } \\
\text { Dose (min) }\end{array}$ & $\underset{(\mathrm{min})}{\mathbf{t}_{\text {int }}}$ & Sequence & $\begin{array}{l}\text { Clinical Outcome } \\
\text { (Comment) }\end{array}$ \\
\hline Vernon et al. [113] & $\begin{array}{c}\text { Localized } \\
\text { superficial } \\
\text { breast cancer, } \\
n=306\end{array}$ & $\begin{array}{c}\text { DHG }^{17}(\mathrm{p}): \\
32.0 / 16 \\
\text { DHG (r): } \\
\text { 40.5-50.0/25 } \\
\text { + boost: } \\
10.0-20.0 \\
\text { MRC } 18 \\
\text { BrR (p): } \\
28.8 / 8 \\
\text { MRC BrI(r) + } \\
\text { MRC BrR(r): } \\
50.0 / 25 \\
\text { + boost: } \\
15.0 / 5 \\
\text { ESHO } 19 \\
32.0 / 8 \\
\text { PMH } \\
\text { PM(p): } \\
32.0 / 18 \\
\text { PMH(r): } \\
50.0 / 25\end{array}$ & 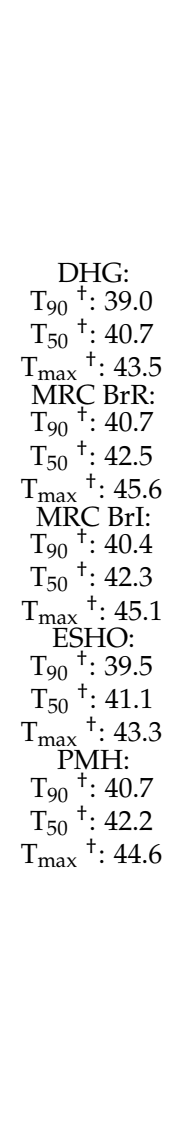 & n.r. & $\begin{array}{c}\text { DHG: } \\
60 \\
(55-61) \\
\text { MRC } \\
\text { BrR: } \\
60 \\
(30-60) \\
\text { MRC } \\
\text { BrI: } \\
60 \\
(17-65) \\
\text { ESHO: } \\
60 \\
(60-60) \\
\text { PMH: } \\
60\end{array}$ & $\begin{array}{c}\text { DGH: } \\
\text { maximum of } \\
\text { CEM42 }{ }^{\circ} \mathrm{C}^{+}: \\
0(0-69.5) \\
\mathrm{CEM} 43^{\circ} \mathrm{C}^{+}: \\
3.95(0-122) \\
\text { MRC: } \\
\text { maximum of } \\
\text { CEM42 }{ }^{\circ} \mathrm{C}^{\dagger}: \\
9(0-60) \\
\mathrm{CEM} 43^{\circ} \mathrm{C}^{+}: \\
7.5(0.1-87.7) \\
\text { ESHO: } \\
\text { maximum of } \\
\mathrm{CEM} 42^{\circ} \mathrm{C}^{+}: \\
5(0-59) \\
\mathrm{CEM} 43^{\circ} \mathrm{C}^{+}: \\
8.4(0.2-74) \\
\text { PMH: } \\
\text { maximum of } \\
\mathrm{CEM} 42^{\circ} \mathrm{C}^{\dagger}: \\
0(0-32.8) \\
\mathrm{CEM} 43^{\circ} \mathrm{C}^{+}: \\
1.5(0-25) \\
\text { data from } \\
\text { Sherar et al. [39] }\end{array}$ & n.r. & n.r. & $\begin{array}{l}\text { - Total CR: 59\%, DHG: } 73.6 \% \\
\text { (14/19), MRC BrI: } 55.5 \% \\
\text { (10/18), MRC BrR: } 56.67 \% \\
(51 / 90), \text { ESHO: } 77.77 \% \\
\text { (21/27), PMH: } 29.41 \% \\
\text { (5/17). } \\
\text { CR rate of previously } \\
\text { non-irradiated: } 61 \% \text { and } \\
\text { CR rate of previously } \\
\text { irradiated tumor: } 46 \% \text {. } \\
\text { 2-year actuarial survival } \\
\text { rate for all patients: } 40 \% . \\
\text { Two largest studies (ESHO } \\
\text { and MRC BrR) showed a } \\
\text { statistically significant } \\
\text { (p=0.004 and 0.001, } \\
\text { respectively) advantage for } \\
\text { the addition of HT, } \\
\text { whereas the other three } \\
\text { trials do not show a benefit } \\
\text { (ORs }<1 \text { ). } \\
\text { CR rate show dependency } \\
\text { on size of tumor, the depth } \\
\text { of the lesion, and on a } \\
\text { history or presence of } \\
\text { metastatic dis-ease outside } \\
\text { the target area (univariate } \\
\text { analysis). } \\
\text { OS did not differ markedly } \\
\text { but patients receiving HT } \\
\text { has a marginally inferior } \\
\text { survival. } \\
\text { Sherar et al. [39]: initial CR } \\
\text { rate is significantly } \\
\text { correlated with thermal } \\
\text { dose and no correlation } \\
\text { between } \mathrm{N}_{\text {total }} \text { and initial } \\
\text { CR rate. }\end{array}$ \\
\hline
\end{tabular}


Table 7. Cont.

\begin{tabular}{|c|c|c|c|c|c|c|c|c|c|}
\hline Author(s) & Cancer Site, $n$ & $\begin{array}{l}\text { RT Dose (Gy) } \\
\text { /Fractions }\end{array}$ & $\begin{array}{l}\text { Temperature } \\
\text { Metrics }\left({ }^{\circ} \mathrm{C}\right)\end{array}$ & $\begin{array}{c}\text { HT } \\
\text { Session }\end{array}$ & $\begin{array}{l}t_{\text {treat }} \\
\text { (min) }\end{array}$ & $\begin{array}{c}\text { Thermal } \\
\text { Dose (min) }\end{array}$ & $\begin{array}{c}t_{\text {int }} \\
(\mathrm{min})\end{array}$ & Sequence & $\begin{array}{c}\text { Clinical Outcome } \\
\text { (Comment) }\end{array}$ \\
\hline Datta et al. [114] & $\begin{array}{l}\text { Head \& neck } \\
\text { cancer, } n=33\end{array}$ & $\begin{array}{l}50.0 \\
/ 25\end{array}$ & n.r. & $\begin{array}{l}\mathrm{N}_{\text {total }}: \\
8-10 \\
\mathrm{~N}_{\text {week }}: \\
\quad 2\end{array}$ & n.r. & n.r. & n.r. & HT before RT & $\begin{array}{l}\text { - } \quad \text { RR: } 76 \% \text {, CR: } 55 \% \text {, PR: } 21 \% \\
\text { and DFS: } 33 \% \text {. } \\
\text { Particularly significant } \\
\text { effect in patients with } \\
\text { advanced disease. } \\
\text { (correlation of thermometric } \\
\text { parameters with clinical outcome } \\
\text { not presented) }\end{array}$ \\
\hline Overgaard et al. [115] & $\begin{array}{c}\text { Recurrent } \\
\text { or metastatic } \\
\text { malignant } \\
\text { melanoma, } n=63\end{array}$ & $\begin{array}{c}24.0-27.0 \\
/ 3\end{array}$ & n.r. & $\begin{array}{c}\mathrm{N}_{\text {total }}: \\
3 \\
\mathrm{~N}_{\text {week }}: \\
1\end{array}$ & 60 & $\begin{array}{l}\mathrm{CEM}^{\mathrm{C}} 43^{\circ} \mathrm{C}^{\dagger} \text { : } \\
9(0-219) \\
\text { data from } \\
\text { Overgaard } \\
\text { et al. [116] }\end{array}$ & 30 & HT after RT & $\begin{array}{l}\text { HT did not significantly } \\
\text { increase acute or late } \\
\text { radiation reactions. } \\
\text { 5-year survival rate was } \\
19 \% \text { and was } 38 \% \text { for the } \\
\text { patients for with control of } \\
\text { all known disease. } \\
\text { RR: } 80 \% \text {, initial CR rate: } \\
62 \%, \text { PR: } 32 \% \text {, NR: } 20 \% \text {, } \\
\text { 2-year actuarial LC: } 37 \% \text {. } \\
\text { The response rate was } \\
\text { higher receiving } 27 \mathrm{~Gy} \\
\text { than those receiving a } \\
\text { lower dose. } \\
\text { Both acute and late adverse } \\
\text { effects were deemed } \\
\text { acceptable. } \\
\text { Overgaard et al. [116]: } \\
\text { there is a significance of } \\
\text { thermal dose relationship } \\
\text { with the heat effect but no } \\
\text { correlation between } \mathrm{N}_{\text {total }} \\
\text { and the outcome } \\
\text { of treatment. }\end{array}$ \\
\hline
\end{tabular}


Table 7. Cont.

\begin{tabular}{|c|c|c|c|c|c|c|c|c|c|}
\hline Author(s) & Cancer Site, $n$ & $\begin{array}{l}\text { RT Dose (Gy) } \\
\text { /Fractions }\end{array}$ & $\begin{array}{l}\text { Temperature } \\
\text { Metrics }\left({ }^{\circ} \mathrm{C}\right)\end{array}$ & $\begin{array}{c}\text { HT } \\
\text { Session }\end{array}$ & $\begin{array}{l}t_{\text {treat }} \\
\text { (min) }\end{array}$ & $\begin{array}{c}\text { Thermal } \\
\text { Dose (min) }\end{array}$ & $\begin{array}{c}\mathrm{t}_{\text {int }} \\
\text { (min) }\end{array}$ & Sequence & $\begin{array}{c}\text { Clinical Outcome } \\
\text { (Comment) }\end{array}$ \\
\hline Dinges et al. [41] & $\begin{array}{l}\text { Uterine cervix } \\
\text { carcinomas, } n=18\end{array}$ & $\begin{array}{c}50.4 / 28 \\
\text { with } \\
\text { HDR-IRT } \\
\text { (192 Ir): } \\
20.0 / 4\end{array}$ & $\begin{array}{c}\mathrm{T}_{20}{ }^{+}: 41.7 \\
(40.3-43.2) \\
\mathrm{T}_{50}{ }^{+}: 41.1 \\
(39.2-42.5) \\
\mathrm{T}_{90}{ }^{+}: 39.9 \\
(37.7-41.9)\end{array}$ & $\begin{array}{c}\mathrm{N}_{\text {total }}: \\
4 \\
\mathrm{~N}_{\text {week }}: \\
2\end{array}$ & 60 & $\begin{array}{c}\mathrm{CEM}^{\circ} 3^{\circ} \mathrm{CT}_{20}{ }^{\dagger} \text { : } \\
48.2(5.9-600.5) \\
\mathrm{CEM}^{\circ} 3^{\circ} \mathrm{CT}_{50}{ }^{+}: \\
15.2(0.6-54.0) \\
\mathrm{CEM}^{\circ} 3^{\circ} \mathrm{CT}_{90}{ }^{+}: \\
6.8(0.4-23.0)\end{array}$ & n.r. & n.r. & 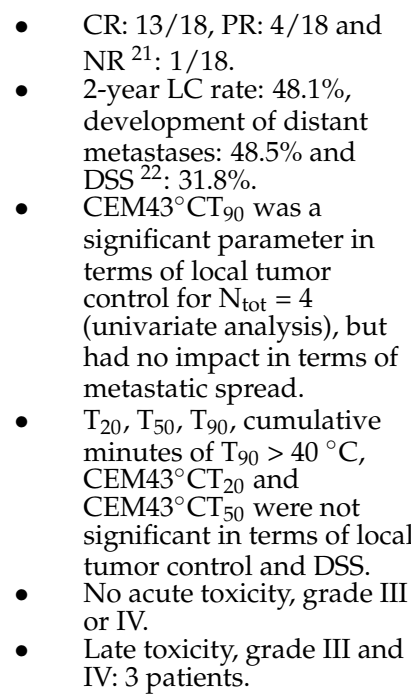 \\
\hline $\begin{array}{l}\text { Kim } \\
\text { et al. [117] }\end{array}$ & $\begin{array}{c}\text { Inoperable } \\
\text { hepatoma, } n=30\end{array}$ & $30.6 / 17$ & n.r. & $\begin{array}{c}\mathrm{N}_{\text {total }}: \\
6 \\
\mathrm{~N}_{\text {week }}: \\
2\end{array}$ & $30-60$ & n.r. & 30 & n.r. & $\begin{array}{l}\text { - } \quad \text { Subjective response rate: } \\
78.6 \% \text {, PR: } 40 \% \text {, stable } \\
\text { disease: } 46.7 \% \text {, PD: } 13.3 \% \text {. } \\
\text { 1-year survival values for } \\
\text { all patients and for the } \\
\text { partial responders were } \\
34 \% \text { and } 50 \% \text {, respectively. } \\
\text { (correlation of thermometric } \\
\text { parameters with clinical outcome } \\
\text { not presented) }\end{array}$ \\
\hline
\end{tabular}


Table 7. Cont.

\begin{tabular}{|c|c|c|c|c|c|c|c|c|c|}
\hline Author(s) & Cancer Site, $n$ & $\begin{array}{l}\text { RT Dose (Gy) } \\
\text { /Fractions }\end{array}$ & $\begin{array}{l}\text { Temperature } \\
\text { Metrics }\left({ }^{\circ} \mathrm{C}\right)\end{array}$ & $\begin{array}{c}\text { HT } \\
\text { Session }\end{array}$ & $\begin{array}{l}t_{\text {treat }} \\
\text { (min) }\end{array}$ & $\begin{array}{l}\text { Thermal } \\
\text { Dose (min) }\end{array}$ & $\begin{array}{c}t_{\text {int }} \\
(\mathrm{min})\end{array}$ & Sequence & $\begin{array}{l}\text { Clinical Outcome } \\
\text { (Comment) }\end{array}$ \\
\hline Engin et al. [98] & $\begin{array}{l}\text { Superficial tumors, } \\
\qquad n=50\end{array}$ & $\begin{array}{c}\text { 60.0-70.0 } \\
\text { /30-35 } \\
\text { when } \\
\text { previouslyirradiated: } \\
40.0 / 10\end{array}$ & $\begin{array}{c}\text { Group A: } \\
\mathrm{T}_{\min }{ }^{+}: \\
39.6 \pm 0.2 \\
\text { Group B: } \\
\mathrm{T}_{\min }{ }^{+}: \\
39.3 \pm 0.2\end{array}$ & $\begin{array}{c}\text { Group A: } \\
\mathrm{N}_{\text {total }} \text { : } \\
4 \\
\mathrm{~N}_{\text {week }} \text { : } \\
1 \\
\text { Group A: } \\
\mathrm{N}_{\text {total }} \text { : } \\
8 \\
\mathrm{~N}_{\text {week }} \text { : } \\
2\end{array}$ & 60 & 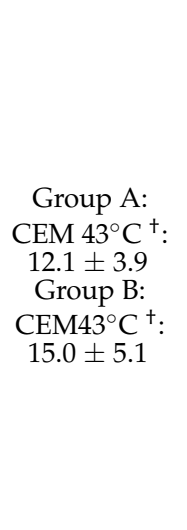 & $15-30$ & HT after RT & $\begin{array}{l}\text { Group A patients treated } \\
\text { with once-weekly HT } \\
\text { session had CR: } 59 \% \\
(12 / 22), \text { PR: } 36 \%(8 / 22), \\
\text { NR: } 5 \%(1 / 22) . \\
\text { Group B patients treated } \\
\text { with twice-weekly HT } \\
\text { sessions had CR: } 55 \% \\
(12 / 22), \text { PR: } 45 \%(10 / 22) . \\
\text { - } \text { T }_{\text {min }} \text { did not influence } \\
\text { response between Group A } \\
\text { and Group B. } \\
\text { Neither tumor response, } \\
\text { duration of LC nor } \\
\text { occurrence of skin } \\
\text { reactions were significantly } \\
\text { affected by N } \mathrm{N}_{\text {week. }}\end{array}$ \\
\hline
\end{tabular}

n: number of patients assigned to be treated with $\mathrm{HT}$ in combination with $\mathrm{RT}^{+}{ }^{+}$: mean value ( \pm standard deviation) or mean value (range); ${ }^{\ddagger}:$ median (range); .r.: not reported; ${ }^{1} \mathrm{CR}$ complete response ${ }^{2} \mathrm{PR}$ : partial response: ${ }^{3} \mathrm{PD}$ : progressive disease; ${ }^{4} \mathrm{LC}$ : local control; ${ }^{5} \mathrm{CEM} 42.5^{\circ} \mathrm{C}$ : cumulative equivalent minutes at reference temperature $42.5{ }^{\circ} \mathrm{C} ;{ }^{6} \mathrm{OS}$ :overall comple 7 NC: no survival, NC: no change; ${ }_{14}$ LRFS: local relapse-free survival; ${ }^{9}$ DFS: disease free survival; ${ }^{10}$ RR: responserate, ${ }^{11}$ LR: local response, ${ }^{12}$ pCR: pathological CR, ${ }_{18}{ }^{2}$ LF: local failure, ${ }^{14}$ DM: distant mester specific antigen; ${ }^{1}$ DHG: Daniel den Hoed Cancer Center in Rotterdam; ${ }^{2}$ MRC: Medical Research Council at the Hammersmith Hospital; ${ }^{19}$ ESHO: European Society of Hyperthermic Oncology; ${ }^{20} \mathrm{PMH}$ : Princess Margaret Hospital/Ontario Cancer Institute; ${ }^{21}$ NR: no response; ${ }^{22}$ DSS: disease specific survival; ${ }^{23}$ HDR-IRT: high dose rate interventional radiotherapy; ${ }^{24}$ LDR-IRT: low dose rate interventional radiotherapy. 
In a phase III study of the International Collaborative Hyperthermia Group, led by Vernon et al. [113], thermal dose was associated with complete response (CR) in patients treated for superficial recurrences of breast cancer [39]. Another randomized study showed that the best tumor control probability was dependent on thermal dose [106]. Further, retrospective analyses indicate that thermal dose is a significant predictor of different clinical endpoints (Table 8) [33,36]. A few studies did not find such significant relationships between clinical endpoints and thermal dose $[103,109,110]$. For example, in the prospective study of Maguire et al., a total $\mathrm{CEM} 43^{\circ} \mathrm{CT}_{90}$ with a threshold above 10 min did not show a significant effect on CR [110]. However, the association of $\mathrm{CEM} 43^{\circ} \mathrm{CT}_{90}$ with $\mathrm{CR}$ was later reported for patients treated with superficial malignant cancers [35]. Similar to the study by Maguire et al., the minimum effective thermal dose was set as $10 \mathrm{CEM}_{4} 3^{\circ} \mathrm{CT}_{90}$. In addition, a test HT session was performed to verify if the tumor was heatable, and a thermal dose of higher than $0.5 \mathrm{CEM} 43^{\circ} \mathrm{CT}_{90}$ could be achieved $[35,110]$. The objective of the study by Hurwitz et al. was to achieve a CEM $43{ }^{\circ} \mathrm{CT}_{90}$ of $10 \mathrm{~min}$, yet the resulting mean of thermal dose for all 37 patients was only $8.4 \mathrm{~min}$ [112]. The cumulative minutes $\mathrm{T}_{90}>40.5^{\circ} \mathrm{C}$, defined as 'the time in minutes with $\mathrm{T}_{90}>40.5^{\circ} \mathrm{C}$ for the whole $\mathrm{N}_{\text {total }}$ ', with a mean of $179 \pm 92 \mathrm{~min}$, together with $\mathrm{T}_{90}$ and $\mathrm{T}_{\max }$ were reported to correlate with toxicity and prostate specific antigen clinical endpoints [99]. Similarly, Leopold et al. showed that cumulative minutes of $\mathrm{T}_{90}>40^{\circ} \mathrm{C}$ is a predictor of treatment endpoints [40]. In retrospective studies, TRISE thermal dose concepts [36] were shown to have a predictive role in treatment response. These retrospective analyses showed that TRISE had a significant effect on local control for a cohort of patients with cervical cancer [33].

The effect of the $t_{\text {int }}$ parameter has been only analyzed with respect to treatment endpoints in retrospective studies. The study by van Leeuwen et al. reported that a $t_{\text {int }}$ less than $79.2 \mathrm{~min}$ between RT and reaching $41^{\circ} \mathrm{C}$ during HT was associated with a lower risk of in-field recurrences (IFR) and a better overall survival (OS) in comparison to a longer $t_{\text {int }}$ [22]. In contrast, another retrospective study showed that neither a shorter $t_{\text {int }}$ of 30-74 min nor a longer $t_{\text {int }}$ of 75-220 min between RT and the start of HT were significant predictors of local control (LC), disease free survival (DFS), disease specific survival (DSS) or OS [33]. Thus, the optimal $t_{\text {int }}$ between HT and RT to achieve a maximal effect on the tumor remains unknown.

Apart from heat-related parameters, the total dose of ionizing radiation and its fractionation in combination with HT has an impact on clinical treatment response [118,119]. Valdagni et al. [103] reported that increasing the total dose of RT appeared to improve clinical response as 71\% (5/7) and 90\% (9/10) CR rates were observed for patients with nodal metastases of head and neck cancers who received total doses of 64-66 Gy or 66.1-70 Gy, respectively. In addition, it was reported that previously irradiated tumors, which are typically more resistant to ionizing radiation, achieved higher CR rates when treated with combined RT and HT in comparison with RT alone [35].

Furthermore, RT technique has been reported to have a beneficial effect on combined RT and HT treatment outcomes [29]. For example, technological advance such as MRIguided brachytherapy were shown to improve the treatment outcome when RT is combined with HT [36].

The weak, and in part contradictory, evidence from clinical studies clearly shows that further analyses of thermometric parameters are required to define reference values for clinical use. The reported values for thermometric parameters from prospective and retrospective clinical studies (Tables 7 and 8) can be translated into standard references after being tested and validated in prospective clinical trials. 
Table 8. Retrospective clinical studies using RT in combination with HT.

\begin{tabular}{|c|c|c|c|c|c|c|c|c|c|}
\hline Author(s) & Cancer Site, $n$ & $\begin{array}{c}\text { RT Dose (Gy) } \\
\text { /Fractions }\end{array}$ & $\begin{array}{l}\text { Temperature } \\
\text { Metrics }\left({ }^{\circ} \mathrm{C}\right)\end{array}$ & $\begin{array}{c}\text { HT } \\
\text { Session }\end{array}$ & $\begin{array}{l}t_{\text {treat }} \\
(\mathrm{min})\end{array}$ & $\begin{array}{l}\text { Thermal } \\
\text { Dose (min) }\end{array}$ & $\begin{array}{c}\mathrm{t}_{\text {int }} \\
(\mathrm{min})\end{array}$ & Sequence & $\begin{array}{l}\text { Clinical Outcome } \\
\text { (Comment) }\end{array}$ \\
\hline Franckena et al. [36] & $\begin{array}{c}\text { Locally } \\
\text { advanced cervix } \\
\text { cancer, } \\
n=420\end{array}$ & $\begin{array}{c}\text { 46.0-50.4 } \\
\text { /23-28 } \\
\text { with } \\
\text { HDR-IRT }{ }^{11} \\
\text { (192 Ir): } \\
17.0 / 2 \\
\text { weekly } \\
\text { or LDR-IRT }{ }^{12} \text { : } \\
18.0 / 3 \\
\text { weekly } \\
\text { or LDR: } \\
30 \text { Gy in } \\
60 \mathrm{~h}\end{array}$ & n.r. & $\begin{array}{c}\mathrm{N}_{\text {total }}: \\
5 \\
\mathrm{~N}_{\text {week }}: \\
1\end{array}$ & 60 & $\begin{array}{l}\mathrm{CEM} 43^{\circ} \mathrm{CT}_{90}{ }^{\dagger}: \\
5.05 \pm 4.18 \mathrm{~min}\end{array}$ & n.r. & n.r. & $\begin{array}{l}\text { - } \mathrm{CR}^{1}: 78 \%, \mathrm{PR}^{2}: 16 \%, \mathrm{SD}^{3}: 3 \% \text {, } \\
\text { PD }{ }^{4}: 1 \% \text {. } \\
\text { 1-year PTC } 5 \text { : } 65 \%(95 \% \text { CI: } 60-70 \%) \text {, } \\
\text { 5-year PTC: } 53 \%(95 \% \text { CI: } 47-58 \%) . \\
\text { 1 year DSS } 6 \text { : } 75 \%(95 \% \text { CI: } 71-79 \%) \\
\text { and 5-year DSS: } 47 \% \\
\text { (95\% CI: } 41-53 \%) . \\
\text { Toxicity of grade I: 51\% (80/153), } \\
\text { grade II: 39\% (60/153), grade III: } \\
\text { 9\%(16/153) and grade IV: 0.6\% } \\
\text { (1/153). } \\
\text { Tumor stage, performance status, } \\
\text { radiotherapy dose and tumor size, } \\
\text { CEM43 }{ }^{\circ} \mathrm{CT}_{90} \text { and TRISE emerged as } \\
\text { significant predictors of the } \\
\text { various end-points. }\end{array}$ \\
\hline
\end{tabular}

- $\quad$ TRISE and CEM43T 90 had a significant effect on LC (univariate and multivariate analyses).

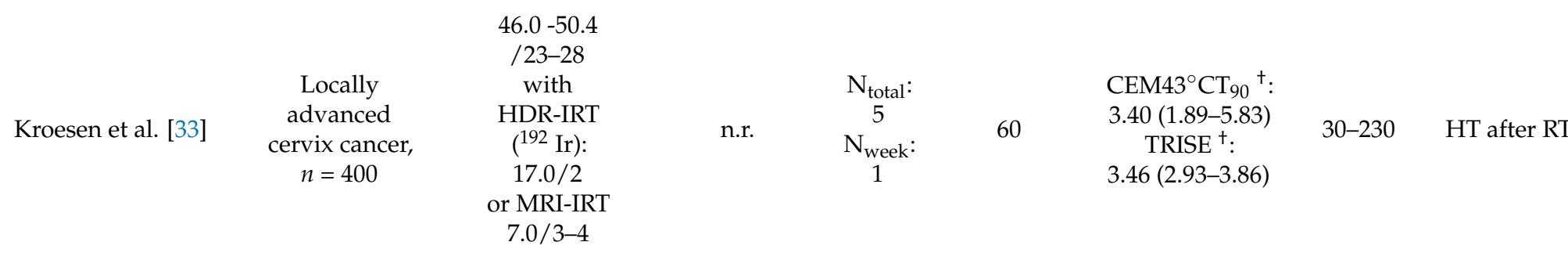

- $\quad$ TRISE, and IGBT showed a significant effect on DFS ${ }^{7}$, DSS, and $\mathrm{OS}^{8}$ (univariate analyses).

- $\quad t_{\text {int }}$ grouped based on median value in short $\mathrm{t}_{\text {int }}(30-74 \mathrm{~min})$ and long $\mathrm{t}_{\text {int }}$ (75-220 $\mathrm{min}$ ) were not significant predictor of LC, DFS, DSS and OS.

- The incidence of late grade III toxicity did not differ between low or high TRISE or low or high $t_{\text {int }}$ patients. 
Table 8. Cont.

\begin{tabular}{|c|c|c|c|c|c|c|c|c|c|}
\hline Author(s) & Cancer Site, $n$ & $\begin{array}{l}\text { RT Dose (Gy) } \\
\text { /Fractions }\end{array}$ & $\begin{array}{l}\text { Temperature } \\
\text { Metrics }\left({ }^{\circ} \mathrm{C}\right)\end{array}$ & $\begin{array}{c}\text { HT } \\
\text { Session }\end{array}$ & $\begin{array}{l}\mathfrak{t}_{\text {treat }} \\
\text { (min) }\end{array}$ & $\begin{array}{l}\text { Thermal } \\
\text { Dose (min) }\end{array}$ & $\begin{array}{c}\mathbf{t}_{\text {int }} \\
(\min )\end{array}$ & Sequence & $\begin{array}{l}\text { Clinical Outcome } \\
\text { (Comment) }\end{array}$ \\
\hline $\begin{array}{c}\text { van Leeuwen } \\
\text { et al. [22] }\end{array}$ & $\begin{array}{c}\text { Locally } \\
\text { advanced } \\
\text { cervix cancer, } \\
n=58\end{array}$ & $\begin{array}{c}46.0-50.4 \\
\text { /23-28 } \\
\text { with } \\
\text { PDR: } \\
24\end{array}$ & n.r. & $\begin{array}{c}\mathrm{N}_{\text {total }}: \\
4-5 \\
\mathrm{~N}_{\text {week }}: \\
1\end{array}$ & 60 & n.r. & $\begin{array}{c}33.8- \\
125.2 \\
+\end{array}$ & HT after RT & $\begin{array}{l}\text { - } \quad \text {-year IFR }{ }^{9}: 18 \%(0-35 \%) \text { in the short } \\
\mathrm{t}_{\text {int }}(\leq 79.2 \mathrm{~min}) \text { group and } 53 \% \\
(18-82 \%) \text { in the long } \mathrm{t}_{\text {int }} \\
\text { ( }>79.2 \mathrm{~min}) \text { group. } \\
\text { - } \quad \text {-year OS: } 52 \%(35-77 \%) \text {. } \\
\text { OS } \ddagger: 61 \text { months }(38-83 \text { months) in } \\
\text { the short } \mathrm{t}_{\text {int }} \text { group and } 19 \text { months } \\
\text { (13-26 months) in the long } \mathrm{t}_{\text {int }} \text { group. } \\
\text { No difference in toxicity was } \\
\text { observed between short and long } \\
\mathrm{t}_{\text {int }} \text { group. }\end{array}$ \\
\hline $\begin{array}{l}\text { Franckena } \\
\text { et al. [120] }\end{array}$ & $\begin{array}{c}\text { Locally } \\
\text { advanced } \\
\text { cervix cancer, } \\
n=378\end{array}$ & $\begin{array}{c}\text { 46.0-50.4 } \\
\text { /23-28 } \\
\text { with } \\
\text { HDR-IRT }(192 \\
\text { Ir): } \\
17.0 / 2 \\
\text { or } \\
18.0-21.0 / 3 \\
\text { or } \\
20.0-24.0 / 1 \\
\text { or } \\
\text { HDR: } \\
\text { 30.0/1 }\end{array}$ & $\mathrm{T}_{\mathrm{avg}}{ }^{\dagger}: 40.6$ & $\begin{array}{c}\mathrm{N}_{\text {total }}: \\
5 \\
\mathrm{~N}_{\text {week }}: \\
1\end{array}$ & 60 & n.r. & $30-240$ & HT after RT & $\begin{array}{ll}\text { - } & \text { CR: } 77 \% \text {. } \\
\text { 5- year PTC: } 53 \% \text { for all patients } \\
\text { (95\% CI, 48-59) and 5-year DSS: } 47 \% \\
\text { (95\% CI, 41-53). } \\
\text { - } \mathrm{N}_{\text {total }} \text { significant influence on CR, } \\
\text { DSS and OS (univariate analysis) and } \\
\text { on CR and DSS } \\
\text { (multivariate analysis). }\end{array}$ \\
\hline
\end{tabular}


Table 8. Cont.

\begin{tabular}{|c|c|c|c|c|c|c|c|c|c|}
\hline Author(s) & Cancer Site, $n$ & $\begin{array}{l}\text { RT Dose (Gy) } \\
\text { /Fractions }\end{array}$ & $\begin{array}{l}\text { Temperature } \\
\text { Metrics }\left({ }^{\circ} \mathrm{C}\right)\end{array}$ & $\begin{array}{c}\text { HT } \\
\text { Session }\end{array}$ & $\begin{array}{l}t_{\text {treat }} \\
(\mathrm{min})\end{array}$ & $\begin{array}{l}\text { Thermal } \\
\text { Dose (min) }\end{array}$ & $\begin{array}{c}\mathrm{t}_{\text {int }} \\
(\mathrm{min})\end{array}$ & Sequence & $\begin{array}{l}\text { Clinical Outcome } \\
\text { (Comment) }\end{array}$ \\
\hline $\begin{array}{l}\text { Oldenborg } \\
\text { et al. [121] }\end{array}$ & $\begin{array}{l}\text { Recurrent breast } \\
\text { cancer, } n=78\end{array}$ & $32.0 / 8$ & $\begin{array}{c}\mathrm{T}_{90}{ }^{+}: 41.1 \\
(37.7-42.4) \\
\mathrm{T}_{50}{ }^{+}: 42.2 \\
(39.0-43.4) \\
\mathrm{T}_{10}{ }^{+}: 43.2 \\
(41.0-44.5)\end{array}$ & $\begin{array}{c}\mathrm{N}_{\text {total }}: \\
4 \\
\mathrm{~N}_{\text {week }} \\
1\end{array}$ & 60 & 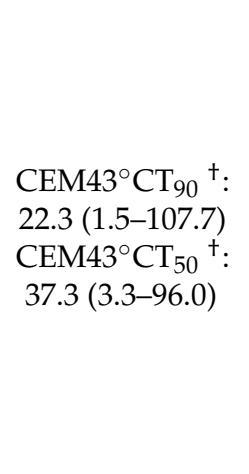 & 60 & HT after RT & $\begin{array}{l}\text { 3- and 5-year OS: } 66 \% \text { and } 49 \% \text {, } \\
\text { respectively. } \\
\text { 3- and 5-year LC: } 78 \% \text { and } 65 \% \text {, } \\
\text { respectively. } \\
\text { The only significant prognostic } \\
\text { factor: time between primary and } \\
\text { recurrent disease (multivariate } \\
\text { analyses) } \\
\text { CEM } 43^{\circ} \mathrm{CT}_{90} \text { was not analyzed } \\
\text { because skin temperature } \\
\text { measurements are poor indicators of } \\
\text { tumor temperature. }\end{array}$ \\
\hline Datta et al. [49] & $\begin{array}{l}\text { Muscle invasive } \\
\text { bladder cancer, } \\
\qquad n=18\end{array}$ & $\begin{array}{c}\text { unifocal } \\
\text { cancer: } \\
48.0 / 16 \\
\text { multifocal } \\
\text { cancer: } \\
50.0 / 20\end{array}$ & $\begin{array}{c}\mathrm{T}_{\text {avg }}{ }^{+}: \\
40.5 \pm 0.5 \\
\mathrm{~T}_{\min }{ }^{+}: \\
36.7 \pm 0.3 \\
\mathrm{~T}_{\max }{ }^{+}: \\
42.0 \pm 0.6\end{array}$ & $\begin{array}{l}\mathrm{N}_{\text {total }}: \\
\quad 4 \\
\mathrm{~N}_{\text {week }}: \\
\quad 1\end{array}$ & 60 & $\begin{array}{l}\mathrm{CEM} 43^{\circ} \mathrm{C}: \\
59.8 \pm 45.6\end{array}$ & $15-20$ & $\begin{array}{l}\text { HT before } \\
\text { RT }\end{array}$ & $\begin{array}{l}\text { - } 16 / 21 \text { patients were free from local } \\
\text { recurrence until their last follow-up } \\
\text { or death. } \\
\text { Temperature attained during } t_{\text {treat }} \\
\text { was significantly lower in patients } \\
\text { with local failure. } \\
\text { AUC }>37^{\circ} \mathrm{C} \text { and AUC } \geq 39^{\circ} \mathrm{C} \text { were } \\
\text { significantly lower in patients who } \\
\text { had a local relapse. } \\
\mathrm{N}_{\text {week and } \mathrm{N}_{\text {total, }} \text { no significant }} \\
\text { differences between } \mathrm{CEM} 43^{\circ} \mathrm{C} \text { and } \\
\mathrm{CEM} 43^{\circ} \mathrm{C} \text { for } \mathrm{T}>37^{\circ} \mathrm{C} \text {. } \\
\mathrm{T}_{\text {avg: significantly greater in patients }} \text { sidith } \\
\text { with no local bladder failure for both } \\
\text { individual and } \mathrm{N}_{\text {total }} \text {. }\end{array}$ \\
\hline
\end{tabular}


Table 8. Cont.

\begin{tabular}{|c|c|c|c|c|c|c|c|c|c|}
\hline Author(s) & Cancer Site, $n$ & $\begin{array}{l}\text { RT Dose (Gy) } \\
\text { /Fractions }\end{array}$ & $\begin{array}{l}\text { Temperature } \\
\text { Metrics }\left({ }^{\circ} \mathrm{C}\right)\end{array}$ & $\begin{array}{c}\text { HT } \\
\text { Session }\end{array}$ & $\begin{array}{l}t_{\text {treat }} \\
(\mathrm{min})\end{array}$ & $\begin{array}{l}\text { Thermal } \\
\text { Dose (min) }\end{array}$ & $\begin{array}{c}t_{\text {int }} \\
(\min )\end{array}$ & Sequence & $\begin{array}{l}\text { Clinical Outcome } \\
\text { (Comment) }\end{array}$ \\
\hline Leopold et al. [32] & $\begin{array}{c}\text { Soft tissue } \\
\text { sarcoma, } n=45\end{array}$ & $\begin{array}{c}50.0-50.4 \\
/ 25-28\end{array}$ & $\begin{array}{c}\mathrm{T}_{90} \ddagger: 39.5 \\
\mathrm{~T}_{50} \neq: 41.6 \\
\mathrm{~T}_{10} \neq: 43.0 \\
\mathrm{~T}_{\min } \ddagger: 37.7 \\
\mathrm{~T}_{\max } \neq: 44.0\end{array}$ & $\begin{array}{l}\text { Group } \\
\text { A: } \\
\mathrm{N}_{\text {total: }} \text { : } \\
5 \\
\mathrm{~N}_{\text {week }} \text { : } \\
1 \\
\text { Group } \\
\text { B: } \\
\mathrm{N}_{\text {total }} \text { : } \\
10 \\
\mathrm{~N}_{\text {week: }} \text { : } \\
2\end{array}$ & 60 & n.r. & $30-60$ & HT after RT & $\begin{array}{l}\text { - Strongest predictive value for } \\
\text { cumulative minimum } \mathrm{T}_{90} \text {, average } \\
\text { min } \mathrm{T}_{90} \text {, cumulative minutes of } \mathrm{T}_{50} \text {, } \\
\text { and average minutes } \mathrm{T}_{50}: 40.5^{\circ} \mathrm{C} \text {, } \\
40.5^{\circ} \mathrm{C}, 41.5^{\circ} \mathrm{C} \text {, and } \\
41.5^{\circ} \mathrm{C} \text {, respectively. } \\
\mathrm{N}_{\text {week }}: 2 \text { were superior to } \mathrm{N}_{\text {week }}: 1 \\
\text { with respect to the degree of } \\
\text { histopathologic changes, but } \\
\text { not predictive. } \\
\text { - } \mathrm{T}_{50} \text { and } \mathrm{T}_{90} \text { are substantially } \\
\text { temperature distribution descriptors. }\end{array}$ \\
\hline Ohguri et al. [94] & $\begin{array}{l}\text { Non-small cell } \\
\text { lung cancer, } \\
n=35\end{array}$ & $\begin{array}{c}45.0-80.0 \\
/ 23-30\end{array}$ & $\begin{array}{c}\mathrm{T}_{\max } \ddagger: 43.2 \\
(38.9-48.1) \\
\mathrm{T}_{\text {avg }} \neq: 42.6 \\
(38.8-47.0) \\
\mathrm{T}_{\min } \neq: 41.7 \\
(38.6-45.6)\end{array}$ & $\begin{array}{c}\mathrm{N}_{\text {total }} \\
11 \\
(3-17) \\
\mathrm{N}_{\text {week }} \text { : } \\
1-2\end{array}$ & $40-70$ & n.r. & 15 & HT after RT & $\begin{array}{l}\text { - } \quad \text { CR: } 2 \%, \text { PR: } 66 \% \text {, and NC } 10: 14 \% \text {. } \\
\text { Median OS, local recurrence-free, } \\
\text { and distant metastasis-free survival } \\
\text { times: } 14.1,7.7 \text {, and } \\
6.1 \text { months, respectively. } \\
\text { Acute toxicity: } 14 \% \text { and late } \\
\text { toxicity: } 17 \% \text {. } \\
\text { All thermal parameters, } \mathrm{T}_{\min }, \mathrm{T}_{\mathrm{avg}} \\
\text { and } \mathrm{T}_{\max } \text { of intraesophageal } \\
\text { temperature significantly correlated } \\
\text { with median } \\
\text { radiofrequency-output power. }\end{array}$ \\
\hline
\end{tabular}

$n$ : number of patients assigned to be treated with $\mathrm{HT}$ in combination with $\mathrm{RT}^{+}{ }^{+}$: mean value ( \pm standard deviation) or mean value (range); ${ }^{\ddagger}:$ median (range); n.r.: not reported; ${ }^{1} \mathrm{CR}$ complete response; ${ }^{2}$ PR: partial response; ${ }^{3}$ SD: stable disease; ${ }^{4}$ PD: progressive disease; ${ }^{5}$ PTC: pelvic tumor control; ${ }^{6}$ DSS: disease specific survival; ${ }^{7}$ DFS: disease free survival; ${ }^{8}$ OS:overall survival; ${ }^{9}$ IFR: in-field recurrence; ${ }^{10}$ NC: no change; ${ }^{11}$ HDR-IRT: high dose rate interventional radiotherapy; ${ }^{12}$ LDR-IRT: low dose rate interventional radiotherapy. 


\section{Evidence for Predictive Values of Thermometric Parameters in Clinical Studies Combining HT and CT}

The added value of combining CT with HT has been established, not only in in vitro and in vivo studies, but also in clinical studies. Randomized clinical studies, which demonstrate that the combination of CT and HT results in improved clinical outcome in comparison with single modality treatment [122-125], confirm the preclinical findings [126]. The positive prospective and retrospective clinical studies are summarized in Tables 9 and 10 respectively, with a focus on thermometric parameters.

The effectiveness of CT drugs has been enhanced by HT in a variety of clinical situations, such as localized, irradiated, recurrent, and advanced cancers, but only few indications are really promising. Long term outcome data, e.g., regarding the combination of CT with HT for bladder cancer, underline the clinical efficacy of this treatment strategy [125]. Chemosensitization by HT is induced by specifics biological interactions between CT drugs and heat. The increased blood flow and the increased fluidity of the cytoplasmic membrane of the cells induced by HT increase the concentration of CT drugs within malignant tissues. Interestingly, Zagar et al. performed a joint analysis of two different clinical trials and reported no significant correlation between drug concentration and combined treatment effect of CT and HT [127]. However, only a few CT drugs with specific properties (Tables 9 and 10) are good candidates to use with HT. Alkylating agents, nitrosureas, platinum drugs, and some antibiotic classes show synergism with HT, whereas only additive effects are reported with pyrimidine antagonists and vinca alkaloids [59]. For example, heat increases the cytotoxicity of cisplatin, as shown by in vitro and in vivo studies [28,55]. Cisplatin concentration increases linearly with temperatures above $38^{\circ} \mathrm{C}$ when applied simultaneously $[28,128]$. Synergy between HT and CT could be obtained at temperatures below $43.5^{\circ} \mathrm{C}$ in a preclinical study [55]. Similarly, enhanced toxicity has been demonstrated for bleomycin [126,129], liposomal doxorubicin [130], and mitomycin-C [131]. Based on the summary of preclinical data, van Rhoon et al. suggested a CEM $43^{\circ} \mathrm{C}$ of $1-15 \mathrm{~min}$ from heating to $40-42{ }^{\circ} \mathrm{C}$ for $30-60 \mathrm{~min}$ for any free $\mathrm{CT} \mathrm{drug}$, including thermos-sensitive liposomal drugs [132].

Lower temperatures might increase the therapeutic window by differential chemosensitization of cancer and normal tissues. In the prospective study of Rietbroek et al. [133] in patients with recurrent cervical cancer treated with weekly cisplatin and HT, three temperature descriptors, $\mathrm{T}_{20}, \mathrm{~T}_{50}$, and $\mathrm{T}_{90}$, including the time in minutes in which $50 \%$ of the measured tumor sites were above $41^{\circ} \mathrm{C}$, indicated a significant difference in these parameters between patients who did and who did not exhibit a CR after treatment. However, there was neither a difference in $\mathrm{T}_{\max }$ between responders and non-responders in a cohort of patients with recurrent soft tissue sarcomas treated with CT and HT [134], nor in a cohort of patients with recurrent cervical cancer [135].

In a prospective study of patients treated with $\mathrm{CT}$ and HT for recurrent ovarian cancer, no significant relationship of $\mathrm{T}_{90}$ and $\mathrm{T}_{50}$ and $\mathrm{CEM} 43^{\circ} \mathrm{CT}_{90}$ and $\mathrm{CEM} 43^{\circ} \mathrm{CT}_{50}$ with clinical outcome was found [136]. Similarly, the independency of $\mathrm{T}_{90}$ and $\mathrm{CEM} 43^{\circ} \mathrm{CT}_{90}$ was also demonstrated in a retrospective study in soft tissue sarcoma [137]. Although a relationship of thermal dose with treatment response has been reported by Vujaskovic et al. [138], the parameters $\mathrm{CEM} 43^{\circ} \mathrm{CT}_{50}$ and $\mathrm{CEM} 43^{\circ} \mathrm{CT}_{90}$ were not statistically different between patients who did or did not respond to the treatment. The low mean value of $\mathrm{T}_{90}=39.7$ (33.5-39.8) ${ }^{\circ} \mathrm{C}$ reported in this study might be the reason for the non-significant relationship of thermal dose with the clinical endpoint in addition to other factors such as hypoxia and vascularization level of the tumor. The first randomized phase III study that assessed the safety and efficacy of CT in combination with HT also recorded a low $\left(\leq 40^{\circ} \mathrm{C}\right)$ mean value of $\mathrm{T}_{90}=39.2^{\circ} \mathrm{C}\left(38.5-39.8^{\circ} \mathrm{C}\right)$. However, the thermometric data were not analyzed or reported in correlation with treatment response [123]. Further investigations are required to understand which temperature is needed to achieve a maximum therapeutic effect, according to the type of CT drug and its concentration. 
Table 9. Prospective clinical studies using CT in combination with HT.

\begin{tabular}{|c|c|c|c|c|c|c|c|c|c|}
\hline Author(s) & $\begin{array}{c}\text { Cancer Site, } \\
n\end{array}$ & $\begin{array}{l}\text { CT Drug(s) } \\
\left(\mathrm{mg} / \mathrm{m}^{2}\right) \times \\
\text { Cycles }\end{array}$ & $\begin{array}{l}\text { Temperature } \\
\text { Metrics }\left({ }^{\circ} \mathrm{C}\right)\end{array}$ & $\begin{array}{c}\text { HT } \\
\text { Session }\end{array}$ & $\begin{array}{c}\mathbf{t}_{\text {treat }} \\
(\mathrm{min})\end{array}$ & $\begin{array}{l}\text { Thermal } \\
\text { Dose }\end{array}$ & $\begin{array}{c}\mathrm{t}_{\text {int }} \\
(\mathrm{min})\end{array}$ & Sequence & $\begin{array}{l}\text { Clinical Outcome } \\
\text { (Comment) }\end{array}$ \\
\hline Issels et al. [123] & $\begin{array}{c}\text { Localised } \\
\text { high-risk } \\
\text { soft-tissue } \\
\text { sarcoma, } n=104\end{array}$ & $\begin{array}{c}125 \text { etoposide } \\
\text { twice weekly } \\
\times 4 \\
1500 \text { ifosfamide } \\
\text { four times } \\
\text { weekly } \\
\times 4 \\
50 \\
\text { doxorubicin } \\
\text { once weekly } \\
\times 4\end{array}$ & $\begin{array}{c}\mathrm{T}_{\max } \ddagger: 41.8 \\
\text { (IQR: } \\
41.1-43.2) \\
\mathrm{T}_{20} \neq: 40.8 \\
\text { (IQR: } \\
40.1-42.3) \\
\mathrm{T}_{50} \ddagger: 40.3 \\
\text { (IQR: } \\
39.5-41.0) \\
\mathrm{T}_{90} \ddagger: 39.2 \\
(\mathrm{IQR}: \\
38.5-39.8)\end{array}$ & $\begin{array}{l}\mathrm{N}_{\text {total }}: \\
8 \\
\mathrm{~N}_{\text {week }}: \\
\quad 2\end{array}$ & 60 & n.r. & n.r. & n.r. & $\begin{array}{l}\text { The proportion of patients who } \\
\text { underwent amputation was } \\
6.7 \%(7 / 104) \text {. } \\
\text { After surgery, } 108 \text { patients } \\
\text { received mean dose of } \\
53.3 \pm 8.9 \text { Gy. } \\
\text { - } 2 \text {-year and } 4 \text {-year LPFS } 1: 58 \% \\
\text { (51-66\%) and } 42 \%(35-51 \%), \\
\text { respectively; } \\
\text { 2-year and } 4 \text {-year OS } 2: 78 \% \\
\text { (72-84\%) and } 59 \%(51-67 \%), \\
\text { respectively; } \\
\text { CR }{ }^{3}, \text { PR }^{4}, \text { SD } 5 \text {, PD }{ }^{6} \text { rates } \\
\text { were } 2.5 \%, 26.3 \%, 55.9 \%, 6.8 \% \text {, } \\
\text { 8.5\%, respectively. } \\
\text { The most frequent } \\
\text { nonhaematological adverse } \\
\text { events, grade III or IV: } \\
23 \text { patients. }\end{array}$ \\
\hline & & & & & & & & & $\begin{array}{l}\text { (correlation of thermometric } \\
\text { parameters with clinical outcome } \\
\text { not presented) }\end{array}$ \\
\hline
\end{tabular}


Table 9. Cont.

\begin{tabular}{|c|c|c|c|c|c|c|c|c|c|}
\hline Author(s) & $\begin{array}{c}\text { Cancer Site, } \\
n\end{array}$ & $\begin{array}{l}\text { CT Drug(s) } \\
\left(\mathrm{mg} / \mathrm{m}^{2}\right) \times \\
\text { Cycles }\end{array}$ & $\begin{array}{l}\text { Temperature } \\
\text { Metrics }\left({ }^{\circ} \mathrm{C}\right)\end{array}$ & $\begin{array}{c}\text { HT } \\
\text { Session }\end{array}$ & $\begin{array}{l}t_{\text {treat }} \\
(\mathrm{min})\end{array}$ & $\begin{array}{c}\text { Thermal } \\
\text { Dose }\end{array}$ & $\begin{array}{c}t_{\text {int }} \\
(\min )\end{array}$ & Sequence & $\begin{array}{l}\text { Clinical Outcome } \\
\text { (Comment) }\end{array}$ \\
\hline $\begin{array}{l}\text { Alvarez Secord } \\
\text { et al. [136] }\end{array}$ & $\begin{array}{c}\text { Refractory } \\
\text { ovarian cancer, } \\
n=30\end{array}$ & $\begin{array}{c}40 \text { doxil } \\
\text { once weekly } \\
\times 6\end{array}$ & $\begin{array}{c}\mathrm{T}_{90}{ }^{+}: \\
39.78 \pm 0.59 \\
\mathrm{~T}_{50}{ }^{+}: 40.47 \pm 0.56\end{array}$ & $\begin{array}{l}\mathrm{N}_{\text {total }}: \\
6\end{array}$ & 60 & $\begin{array}{c}\mathrm{CEM}^{\circ} 3^{\circ} \mathrm{CT}_{90}{ }^{\dagger}: \\
5.84 \pm 5.66 \\
\mathrm{CEM}^{\circ} 3^{\circ} \mathrm{CT}_{50}{ }^{\dagger}: \\
13.00 \pm 11.25\end{array}$ & $0-60$ & HT after $\mathrm{CT}$ & $\begin{array}{l}\text { - PR: } 10 \%(3 / 30), \text { SD: } 27 \%(8 / 30) \text {, } \\
\text { PD: } 63 \%(19 / 30) . \\
\text { - Median of PFS }{ }^{7}: 3.4 \text { and OS: } \\
\text { - } \quad \text { Toxicity due to HT, grade III: } \\
\text { one patient. } \\
\text { No significant differences } \\
\text { between the } \mathrm{T}_{90}, \mathrm{~T}_{50} \\
\mathrm{CEM}^{\circ} 3^{\circ} \mathrm{CT}_{90} \text { or CEM } 43^{\circ} \mathrm{CT}_{50} \\
\text { and those patients who had PD } \\
\text { compared to SD or PR. } \\
\text { No significant change in overall } \\
\text { QoL was found between } \\
\text { baseline and after treatment. }\end{array}$ \\
\hline $\begin{array}{c}\text { Fiegl } \\
\text { et al. [134] }\end{array}$ & $\begin{array}{l}\text { Advanced soft } \\
\text { tissue sarcoma, } \\
n=20\end{array}$ & $\begin{array}{l}1500 \text { ifosfamide } \\
\text { four times } \\
\text { weekly } \\
\times 7 \\
100 \text { carboplatin } \\
\text { four times } \\
\text { weekly } \\
\quad \times 7 \\
150 \text { etoposide } \\
\text { four times } \\
\text { weekly } \\
\quad \times 7\end{array}$ & $\begin{array}{c}\mathrm{T}_{\max }{ }^{\dagger}: 40.6 \\
(39.1-42.2)\end{array}$ & $\begin{array}{c}\mathrm{N}_{\text {total }}: \\
8 \\
\mathrm{~N}_{\text {week }}: \\
2\end{array}$ & 60 & n.r. & n.r. & n.r. & $\begin{array}{l}\text { - } \quad \text { Time } \ddagger \text { to progression: } 6 \text { and to } \\
\text { OS: } 14.6 \text { months. } \\
\text { 3- and 6-months PFR } 8 \\
\text { estimates: } 60 \% \text { and } 45 \% \text {, } \\
\text { respectively. } \\
\text { Grade III/IV haematological } \\
\text { toxicities during CT: } 70 \% \text {. } \\
\text { - Objective RR } 9: 20 \% \text { PR: } 20 \% \\
\text { (4/20), PD: } 45 \%(9 / 20) ; \\
\text { No difference in } \mathrm{T}_{\text {max }} \text { between } \\
\text { responders or non-responders. }\end{array}$ \\
\hline
\end{tabular}


Table 9. Cont.

\begin{tabular}{|c|c|c|c|c|c|c|c|c|c|}
\hline Author(s) & $\begin{array}{c}\text { Cancer Site, } \\
n\end{array}$ & $\begin{array}{l}\text { CT Drug(s) } \\
\left(\mathrm{mg} / \mathrm{m}^{2}\right) \times \\
\text { Cycles }\end{array}$ & $\begin{array}{l}\text { Temperature } \\
\text { Metrics }\left({ }^{\circ} \mathrm{C}\right)\end{array}$ & $\begin{array}{c}\text { HT } \\
\text { Session }\end{array}$ & $\begin{array}{l}t_{\text {treat }} \\
(\mathrm{min})\end{array}$ & $\begin{array}{l}\text { Thermal } \\
\text { Dose }\end{array}$ & $\underset{\text { (min) }}{t_{\text {int }}}$ & Sequence & $\begin{array}{l}\text { Clinical Outcome } \\
\text { (Comment) }\end{array}$ \\
\hline $\begin{array}{l}\text { Rietbroek } \\
\text { et al. [133] }\end{array}$ & $\begin{array}{c}\text { Irradiated } \\
\text { recurrent } \\
\text { cervical cancer, } \\
n=23\end{array}$ & $\begin{array}{l}50 \text { cisplatin } \\
\text { once weekly } \\
\quad \times 12\end{array}$ & $\begin{array}{l}\mathrm{T}_{20}{ }^{+}: 41.9 \pm 0.9^{\circ} \mathrm{C} \\
\mathrm{T}_{50}{ }^{+}: 41.3 \pm 0.8^{\circ} \mathrm{C} \\
\mathrm{T}_{90}{ }^{+}: 40.5 \pm 0.7^{\circ} \mathrm{C}\end{array}$ & $\begin{array}{c}\mathrm{N}_{\text {total }}: \\
12 \\
\mathrm{~N}_{\text {week }} \\
1\end{array}$ & 60 & n.r. & 30 & HT after CT & $\begin{array}{l}\text { - } \quad \text { RR: } 52 \% \text { observed after a } \\
\text { median number of } 8 \text { cycles of } \\
\text { treatment. } \\
\text { - } \text { OS }^{\ddagger} \text { rate: } 8 \text { months, specifically } \\
\text { for responders: } 12 \text { months. } \\
\text { - } \quad \mathrm{T}_{20}, \mathrm{~T}_{50}, \mathrm{~T}_{90} \text { values were higher } \\
\text { for responders than } \\
\text { non-responders but it did not } \\
\text { show a statistical significance. }\end{array}$ \\
\hline Zagar et al. [127] & $\begin{array}{c}\text { Recurrent } \\
\text { breast cancer, } \\
n_{\text {trial } 1}=18 \\
n_{\text {trial } 2}=11\end{array}$ & $\begin{array}{c}\text { Trial A: } \\
\text { 20-60 LTDL } 13 \\
\text { every } 21-35 \\
\text { days } \\
\times 6 \\
\text { Trial B: } \\
\text { 40-50 LTDL } \\
\text { every } 21-35 \\
\text { days } \\
\times 6\end{array}$ & $\begin{array}{l}\max T_{90}: 42.6 \\
\min T_{90}: 36.0\end{array}$ & $\begin{array}{l}\mathrm{N}_{\text {total }}: \\
\quad 6\end{array}$ & $60 \mathrm{~min}$ & n.r. & $30-60$ & HT after $\mathrm{CT}$ & $\begin{array}{l}\text { - Combined trials (A and B), CR: } \\
17.2 \%(5 / 29) \text { and } \\
\text { PR: } 31 \%(9 / 29) . \\
\text { Patients with at least one or } \\
20 \% \text { of HT sessions with a } \mathrm{T}_{90} \\
\text { of target below } 39^{\circ} \mathrm{C} \text { had } \\
\text { similar local objective RR. } \\
\text { Toxicity, grade IV: three } \\
\text { patients }(10.3 \%) \text { and grade III: } \\
\text { six patients ( } 20.7 \%) . \\
\text { No drug dose response } \\
\text { relationship was observed } \\
\text { between trial A and B. } \\
\text { (correlation of thermometric } \\
\text { parameters with clinical outcome } \\
\text { not presented) }\end{array}$ \\
\hline
\end{tabular}


Table 9. Cont.

\begin{tabular}{|c|c|c|c|c|c|c|c|c|c|}
\hline Author(s) & $\begin{array}{c}\text { Cancer Site, } \\
n\end{array}$ & $\begin{array}{l}\text { CT Drug(s) } \\
\left(\mathrm{mg} / \mathrm{m}^{2}\right) \times \\
\text { Cycles }\end{array}$ & $\begin{array}{l}\text { Temperature } \\
\text { Metrics }\left({ }^{\circ} \mathrm{C}\right)\end{array}$ & $\begin{array}{c}\text { HT } \\
\text { Session }\end{array}$ & $\begin{array}{l}t_{\text {treat }} \\
(\mathrm{min})\end{array}$ & $\begin{array}{c}\text { Thermal } \\
\text { Dose }\end{array}$ & $\begin{array}{c}t_{\text {int }} \\
(\mathrm{min})\end{array}$ & Sequence & $\begin{array}{l}\text { Clinical Outcome } \\
\text { (Comment) }\end{array}$ \\
\hline $\begin{array}{l}\text { Ishikawa } \\
\text { et al. [139] }\end{array}$ & $\begin{array}{c}\text { Locally } \\
\text { advanced or } \\
\text { metastatic } \\
\text { pancreatic } \\
\text { cancer, } \\
n=18\end{array}$ & $\begin{array}{c}1000 \\
\text { gemcitabine } \\
\text { once weekly } \\
\times 12\end{array}$ & n.r. & $\begin{array}{c}\mathrm{N}_{\text {total }}: \\
20 \\
\mathrm{~N}_{\text {week }}: \\
1\end{array}$ & 40 & n.r. & $0-1440$ & $\begin{array}{l}\text { HT before } \\
\text { CT }\end{array}$ & $\begin{array}{l}\text { Major grade III-IV adverse } \\
\text { events were neutropenia and } \\
\text { anemia, no sepsis. } \\
\text { Objective RR: and disease } \\
\text { control rates were } 11.1 \% \text { and } \\
61.1 \% \text {, respectively. } \\
\text { OS } ¥: 8 \text { months, and the } 1 \text {-year } \\
\text { survival rate was } 33.3 \% \text {. } \\
\text { (correlation of thermometric } \\
\text { parameters with clinical outcome } \\
\text { not presented) }\end{array}$ \\
\hline $\begin{array}{l}\text { Vujaskovic } \\
\text { et al. [138] }\end{array}$ & $\begin{array}{c}\text { Locally } \\
\text { advanced breast } \\
\text { cancer, } \\
n=43\end{array}$ & $\begin{array}{c}30-75 \text { LTDL } \\
\times 4 \\
100-175 \\
\text { paclitaxel } \\
\times 4\end{array}$ & $\begin{array}{c}\mathrm{T}_{90}{ }^{+}: \\
39.7(37.7-41.8)\end{array}$ & $\begin{array}{c}\mathrm{N}_{\text {total }}: \\
4 \\
\mathrm{~N}_{\text {week }}: \\
2\end{array}$ & 60 & $\begin{array}{l}\mathrm{CEM}^{\circ} 3^{\circ} \mathrm{CT}_{90}{ }^{\dagger}: \\
11.5(1.5-159.3)\end{array}$ & 60 & HT after CT & $\begin{array}{l}\text { - CR: } 9 \%(4 / 43) \text { and pathologica } \\
\text { CR: } 60 \%(26 / 43) ; \\
\text { - } \quad \text {-year DFS }{ }^{10} \text { and OS: } 63 \% \text { and } \\
75 \% \text {, respectively. } \\
\text { - } \quad \text { CEM } 43^{\circ} \mathrm{CT}_{90}{ }^{+} \text {in responders } \\
\text { was significantly greater than } \\
\text { non-responders, } 28.6 \text { and } \\
\text { - } 10.3 \text { min, respectively. } \\
\text { Patients had grade III and } \\
\text { IV toxicity } \\
\text { - } \text { No statistical difference in the } \\
\text { CEM } 43^{\circ} \mathrm{CT}_{50} \text { and } \mathrm{CEM} 43^{\circ} \mathrm{CT}_{90} \\
\text { between treatment responders } \\
\text { and non-responders. }\end{array}$ \\
\hline
\end{tabular}


Table 9. Cont.

\begin{tabular}{|c|c|c|c|c|c|c|c|c|c|}
\hline Author(s) & $\begin{array}{c}\text { Cancer Site, } \\
n\end{array}$ & $\begin{array}{l}\text { CT Drug(s) } \\
\left(\mathrm{mg} / \mathrm{m}^{2}\right) \times \\
\text { Cycles }\end{array}$ & $\begin{array}{l}\text { Temperature } \\
\text { Metrics }\left({ }^{\circ} \mathrm{C}\right)\end{array}$ & $\begin{array}{c}\text { HT } \\
\text { Session }\end{array}$ & $\begin{array}{c}t_{\text {treat }} \\
(\mathrm{min})\end{array}$ & $\begin{array}{l}\text { Thermal } \\
\text { Dose }\end{array}$ & $\begin{array}{c}\mathrm{t}_{\text {int }} \\
(\mathrm{min})\end{array}$ & Sequence & $\begin{array}{l}\text { Clinical Outcome } \\
\text { (Comment) }\end{array}$ \\
\hline $\begin{array}{l}\text { de Wit } \\
\text { et al. [135] }\end{array}$ & $\begin{array}{l}\text { Recurrent } \\
\text { uterine cervical } \\
\text { carcinoma, } \\
n=19\end{array}$ & $\begin{array}{c}60,70,80 \\
\text { cisplatin } \\
\text { once weekly } \\
\times 6\end{array}$ & $\begin{array}{c}\mathrm{T}_{\max }{ }^{\dagger}: 41.6 \pm 0.7 \\
(39.7-43.6)\end{array}$ & $\begin{array}{c}\mathrm{N}_{\text {total }}: \\
6 \\
\mathrm{~N}_{\text {week }}: \\
1\end{array}$ & 60 & n.r. & 0 & HT after $\mathrm{CT}$ & $\begin{array}{l}\text { - No dose limiting toxicity at the } \\
80 \mathrm{mg} / \mathrm{m}^{2} \text { dose level } \\
\text { of cisplatin. } \\
\text { CR: } 1 \text { patient (dose level } \\
80 \mathrm{mg} / \mathrm{m}^{2} \text { ), PR: } 18 \text { patients, SD: } \\
18 \text { patients and PD: } 3 \text { patients } \\
\text { (dose level: } 60-80 \mathrm{mg} / \mathrm{m}^{2} \text { ) and } \\
\text { OS } \ddagger: 54 \% \text {. } \\
\text { The improvement rate in } \\
\text { QoL }{ }^{11}: 82.5 \% \\
\text { No differences between } \\
\text { responders and non-responders } \\
\text { for tumor: contact } \\
\text { temperatures, indicative } \\
\text { temperatures, tumor volume, } \\
\text { oral temperature increase or } \\
\text { total power applied. }\end{array}$ \\
\hline
\end{tabular}

$30 *$ bleomycin

twice weekly

Sugimach

et al. [124]
Oesophageal

$>$ carcinoma,

$n=20$

\begin{abstract}
$\times 3$
\end{abstract}
$50 *$ cisplatin

once weekly

$\times 3$ n.r.

$$
\begin{gathered}
\mathrm{N}_{\text {total }} \text { : } \\
6 \\
\mathrm{~N}_{\text {week }} \text { : } \\
2
\end{gathered}
$$
response: $20 \%, \mathrm{NC}^{12}$ : $50 \%$ and decrease of tumor size in comparison to CT treatment only.

\section{(correlation of thermometric} parameters with clinical outcome not presented)

$n$ : number of patients assigned to be treated with $\mathrm{HT}$ in combination withCT; ${ }^{\dagger}:$ mean value ( \pm standard deviation) or mean value (range); ${ }^{\ddagger}:$ median (range); n.r.: not reported; ${ }^{*}$ : in mg unit only; ${ }^{1}$ LPFS: local progressionfree survival; ${ }^{2}$ OS: overall survival; ${ }^{3}$ CR: complete response; ${ }^{4}$ PR: partial response; ${ }^{5}$ SD: stable disease; ${ }^{6}$ PD: progressive disease; ${ }^{7}$ PFS: progression free survival; ${ }^{8}$ PFR: progression free rate; ${ }^{9}$ RR: response rate; ${ }^{10}$ DFS: disease free survival; ${ }^{11}$ QoL: quality of life; ${ }^{12}$ NC: no change; ${ }^{13}$ LTDL: low temperature liposomal doxorubicin. 
Table 10. Retrospective clinical trial studies using CT in combination with HT.

\begin{tabular}{|c|c|c|c|c|c|c|c|c|c|}
\hline Author(s) & Cancer Site, $n$ & $\begin{array}{l}\text { CT Drug(s) } \\
\left(\mathrm{mg} / \mathrm{m}^{2}\right) \times \\
\text { Cycles }\end{array}$ & $\begin{array}{l}\text { Temperature } \\
\text { Metrics }\left({ }^{\circ} \mathrm{C}\right)\end{array}$ & $\begin{array}{c}\text { HT } \\
\text { Session }\end{array}$ & $\begin{array}{c}\mathbf{t}_{\text {treat }} \\
\text { (min) }\end{array}$ & $\begin{array}{l}\text { Thermal } \\
\text { Dose }\end{array}$ & $\begin{array}{c}\mathbf{t}_{\text {int }} \\
(\mathrm{min})\end{array}$ & Sequence & $\begin{array}{l}\text { Clinical Outcome } \\
\text { (Comment) }\end{array}$ \\
\hline Yang et al. [140] & $\begin{array}{c}\text { Advanced } \\
\text { non-small } \\
\text { cell lung } \\
\text { cancer, } n=48\end{array}$ & $\begin{array}{c}1000 \\
\text { gemcitabine } \\
\text { twice weekly } \\
\times 6 \\
75 \text { cisplatin } \\
\text { twice weekly } \\
\times 6\end{array}$ & n.r. & $\begin{array}{l}\mathrm{N}_{\text {total }}: \\
8 \\
\mathrm{~N}_{\text {week }}: \\
\quad 2\end{array}$ & $40-60$ & n.r. & n.r. & $\begin{array}{c}\text { HT after CT } \\
\text { or } \\
\text { HT before CT }\end{array}$ & $\begin{array}{l}\text { - } \quad \text { No CR }{ }^{1} \text { reported, } \mathrm{PR}^{2} \text { : } \\
37.5 \%(18 / 23), \mathrm{SD}^{3}: \\
33.3 \%(16 / 23), \mathrm{PD}^{4}: \\
29.2 \%(14 / 23) . \\
\text { - } \quad \text { ORR } 5: 37.5 \% \text { and } \\
\text { DCR }{ }^{6}: 70.8 \% . \\
\text { - } 1 \text {-and } 2 \text {-year survival } \\
\text { rates: } 14 \% \text { and } 1.3 \% \text {, } \\
\text { respectively. } \\
\text { Toxicity, grade III: } 14 \\
\text { patients and grade IV: } \\
\text { no patients. } \\
\text { (correlation of thermometric } \\
\text { parameters with clinical } \\
\text { outcome not presented) }\end{array}$ \\
\hline $\begin{array}{l}\text { Tschoep- } \\
\text { Lechner } \\
\text { et al. [141] }\end{array}$ & $\begin{array}{c}\text { Advanced } \\
\text { pancreatic } \\
\text { cancer, } \\
n=23\end{array}$ & $\begin{array}{c}1000 \\
\text { gemcitabine } \\
\text { once weekly } \\
\times 8 \\
25 \text { cisplatin } \\
\text { twice weekly } \\
\times 8\end{array}$ & $\begin{array}{c}\mathrm{T}_{\max }{ }^{\dagger}: 42.1 \\
(40.9-44.1)\end{array}$ & $\begin{array}{c}\mathrm{N}_{\text {week }}: \\
2 \\
\mathrm{~N}_{\text {total }} \\
8:\end{array}$ & 60 & n.r. & 0 & simultaneously & $\begin{array}{l}\text { - } \quad \text { PR: } 4.34 \%(1 / 23), \text { SD: } \\
30.4 \%(7 / 23), \text { PD: } 34.7 \% \\
(8 / 23) ; \\
\text { OS } 7 \neq: 12.9 \text { months } \\
\text { (CI: } 9.9-15.9 \text { months). } \\
\text { - Mild (grade } 1 \text { and } 2) \\
\text { position-related pain } \\
\text { during HT treatment. } \\
\text { (correlation of thermometric } \\
\text { parameters with clinical } \\
\text { outcome not presented) }\end{array}$ \\
\hline
\end{tabular}


Table 10. Cont.

\begin{tabular}{|c|c|c|c|c|c|c|c|c|c|}
\hline Author(s) & Cancer Site, $n$ & $\begin{array}{l}\text { CT Drug(s) } \\
\left(\mathrm{mg} / \mathrm{m}^{2}\right) \times \\
\text { Cycles }\end{array}$ & $\begin{array}{l}\text { Temperature } \\
\text { Metrics }\left({ }^{\circ} \mathrm{C}\right)\end{array}$ & $\begin{array}{c}\text { HT } \\
\text { Session }\end{array}$ & $\begin{array}{c}t_{\text {treat }} \\
(\mathrm{min})\end{array}$ & $\begin{array}{l}\text { Thermal } \\
\text { Dose }\end{array}$ & $\underset{(\mathrm{min})}{\mathbf{t}_{\text {int }}}$ & Sequence & $\begin{array}{l}\text { Clinical Outcome } \\
\text { (Comment) }\end{array}$ \\
\hline Stahl et al. [137] & $\begin{array}{c}\text { Soft tissue } \\
\text { sarcomas, } n=46\end{array}$ & $\begin{array}{c}250 \text { etoposide } \\
\times 4 \\
6000 \\
\text { ifosfamide } \\
\times 4 \\
50 \text { adriamycin } \\
\times 4\end{array}$ & $\begin{array}{c}\mathrm{T}_{90}{ }^{+}: \\
39.90 \pm 0.74 \\
\text { (good } \\
\text { responders) } \\
\text { and } \\
\mathrm{T}_{90}{ }^{+}: \\
39.42 \pm 1.78 \\
\text { (bad } \\
\text { responders) }\end{array}$ & $\begin{array}{c}\mathrm{N}_{\text {week }}: \\
2 \\
\mathrm{~N}_{\text {total }} \\
8\end{array}$ & 60 & 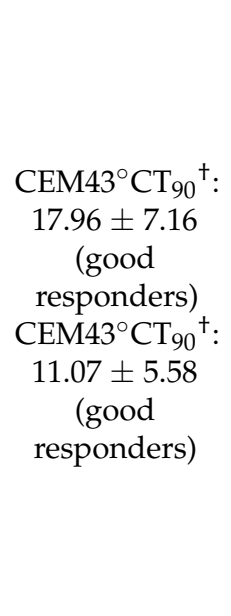 & 0 & simultaneously & $\begin{array}{l}\text { PR: } 31.6 \%(6 / 19 \text { in the } \\
\text { good responder group } \\
\left.\text { for RECIST }{ }^{8}\right) \text { to } 37 \% \\
(10 / 27 \text { in the poor } \\
\text { responder group } \\
\text { for RECIST). } \\
\text { SD: } 63.2 \%(12 / 19 \text { for the } \\
\text { good responder group } \\
\text { in } \mathrm{WHO}^{9} \text { and volume) } \\
\text { to } 70.3 \%(19 / 27 \text { in the } \\
\text { poor responder group } \\
\text { for volume). } \\
\text { T90 and CEM } 43^{\circ} \mathrm{CT}_{90} \\
\text { parameters did not } \\
\text { differ significantly } \\
\text { between the groups. }\end{array}$ \\
\hline
\end{tabular}

$n$ : number of patients assigned to be treated with $\mathrm{HT}$ in combination withCT; ${ }^{+}:$mean value ( \pm standard deviation) or mean value (range); ${ }^{\ddagger}:$ median (range) ${ }^{1} \mathrm{CR}:$ complete response 2 PR: partial response; ${ }^{3}$ SD: stable disease; ${ }^{4}$ PD: progression disease; ${ }^{5}$ ORR: objective response rate; ${ }^{6}$ DCR: disease control rate; ${ }^{7}$ OS: overall survival; ${ }^{8}$ RECIST: Response Evaluation Criteria in Solid Tumors; ${ }^{9}$ WHO: world health organization 
Based on preclinical studies, the delivery of simultaneous CT and HT is recommended to achieve the greatest chemosensitization effect by HT $[55,142]$. However, in contrast to experimental results [20,55], most of the prospective studies listed in Table 9 were designed to deliver heat sequentially, and in most studies the CT drugs were administered prior to HT. Despite the fact that a considerable supra-additive or synergistic effect can be achieved by the simultaneous delivery of CT and RT, the sequential application of CT and HT may protect normal tissues from chemosensitization. The cell killing of hypoxic and oxygenated tumor cells can still be obtained with sequential delivery of CT drugs and HT [54]. In clinical studies, the $t_{\text {int }}$ between modalities is usually kept under an hour $[122,127,133,136,138]$. Of note, the study of Ishikawa et al. showed a different scheduling of gemcitabine and HT for the treatment of locally advanced or metastatic pancreatic cancer [139]. Patients enrolled in this clinical study were treated with HT prior to CT with a $t_{\text {int }}$ of $0-24 \mathrm{~h}$. This unique flexible relationship of gemcitabine cytotoxicity with the $t_{\text {int }}$ and sequence was revealed in an in vitro study [143]. The specific properties of CT drugs are main factors in determining the most efficient treatment sequence between CT and HT for each class of drugs.

That treatment protocols might require individualized standards for HT thermometric parameters as has recently been illustrated by an interim analysis of cisplatin and etoposide given concurrently with HT for treatment of patients with esophageal carcinoma. This analysis showed a relationship between tumor location and temperature reporting, i.e., higher temperatures were achieved in distal tumors [144]. Similar treatment site-dependent analysis of thermometric parameters should be performed in future trials. Although the biology underlying the interaction between CT drugs and heat in cancer and normal tissues is largely unknown, thermometric parameters have been shown to predict outcome when HT is combined with CT. Therefore, as discussed above, no definitive conclusions can be drawn regarding the optimal thermometric parameters for an enhanced effect of HT with CT.

\section{Evidence for Predictive Values of Thermometric Parameters in Clinical Studies Using RT and CT in Combination with HT}

Clinical malignancies, in particular advanced and inoperable tumors, can be treated using triplet therapy consisting of CT, RT and HT as a maximal treatment approach. The number of prospective and retrospective clinical studies investigating this approach is limited, the most important of which are listed in Tables 11 and 12, respectively. These studies have already reported the feasibility of this trimodal approach for cervical cancer, rectal cancer, and pancreatic cancer.

The optimal combination of CT, RT, and HT in a single framework is complex, be-cause so many biological processes underly the interactions between the three modalities. In addition, clinical factors often influence the optimal combination of RT and CT. A template with fundamental specifications for designing a clinical study with the trimodal treatment is proposed by Herman et al. [145].

Even though there is no consensus as to the optimal scheduling of trimodal treatment, clinical studies to date integrate HT in combination with daily RT and CT drugs based on the concept that CT should interact with both RT and HT. Scheduling CT weekly is most feasible in terms of maintaining an optimal $t_{\text {int }}$ between HT sessions, drug administration, and RT fraction [145].

The reason why cisplatin is most frequently used in trimodality regimens is less based on a specific interaction with heat, but rather on extensive evidence from phase III randomized trials showing that cisplatin potently improves the antitumor efficacy of radiotherapy, albeit at the cost of increased toxicity. Drug concentration has been shown to affect treatment response [146], as proven experimentally [147]. A phase I-II study reported that a higher cisplatin dose $\left(50 \mathrm{mg} / \mathrm{m}^{2}\right)$ in comparison with a lower dose $\left(20-40 \mathrm{mg} / \mathrm{m}^{2}\right)$ combined with RT and HT was positively correlated with CR [146]. Interestingly, overall survival between patients treated with two different $\mathrm{CT}$ regimes in combination with RT and HT did not differ [148]. However, the study was limited by the small size of the patient 
cohort. With reference to Table 11 , clinical studies using trimodality treatment usually used conventional fractionation schemes with 1.8-2.0 Gy per fractions, leaving it largely unknown whether other schedules such as hypofractionation (>10 Gy per week or large single fractions) might be biologically more favorable. The total dose varied according to cancer type. In the case of cervical cancer, brachytherapy at high dose rate (HDR) or low dose rate (LDR) was applied to deliver the boost dose $[149,150]$. Furthermore, high or low total RT dose was reported to have an influence on CR rate when combined with 5-FU, leucovorin and HT [151]. In contrast to CT and RT treatment parameters, HT treatment parameters were frequently not reported. Thermometric parameters, such as temperature and thermal dose including $t_{i n t}$, are reported but not set as fixed treatment requirements as there are no accepted reference values.

Disregarding the Arrhenius relationship of heating temperature and $t_{\text {treat }}$, Amichetti et al. [152] reported a short $t_{\text {treat }}$ of $30 \mathrm{~min}$ with mean temperature range values of $\mathrm{T}_{\max }=43.2^{\circ} \mathrm{C}\left(41.5-44.5^{\circ} \mathrm{C}\right)$ and $\mathrm{T}_{\min }=40.1^{\circ} \mathrm{C}\left(37-42^{\circ} \mathrm{C}\right)$. This might explain why this study did not result in a higher $\mathrm{CR}$ rate in comparison to the previous study by Valdagni et al. [103]. A correlation of achieved temperature with treatment response such as disease-free interval to local relapse (DFILR) was reported in the study by Kouloulias et al. [153]. This study showed that the DFILR rate was greater in patients who achieved heating temperature $\mathrm{T}_{90}>44{ }^{\circ} \mathrm{C}$ for longer than 16 min during HT treatment. No significant correlation of DFILR with mean values of temperature descriptor $T_{\min }$ was confirmed. Referring to the last row in Tables 7-12, the clinical endpoints among studies differ, which adds another level of complexity to generalizing the thermometric parameter correlations reported in studies.

Thermal dose was reported less frequently than temperature measurements, hence there is a lack of information about its predictive role for treatment response. In one study, thermal dose was directly and proportionally associated with $\mathrm{CR}$, as patients who exhibited $\mathrm{CR}$ after treatment with a measured $\mathrm{CEM} 43^{\circ} \mathrm{CT}_{90}$ of $4.6 \mathrm{~min}$ in comparison with patients with a PR and a CEM $43^{\circ} \mathrm{CT}_{90}$ of only $2.0 \mathrm{~min}$ [146]. Recently, a prospective phase II study investigating neoadjuvant triplet therapy in patients with rectal cancer showed that patients achieving good local tumor regression had received a high thermal dose [154]. However, no threshold, only the mean of CEM $43{ }^{\circ} \mathrm{C}$, was reported. The retrospective analysis of thermometric parameters of the prospective study by Harima et al. [149] showed that $>1$ min $\mathrm{CEM} 43^{\circ} \mathrm{CT}_{90}$ is the threshold value which significantly correlates with treatment response (CR and disease-free survival rates). It also confirmed that $\mathrm{CEM}_{4} 3^{\circ} \mathrm{CT}_{90}$ below $1 \mathrm{~min}$ are insufficient to achieve enhancement of RT and CT [155]. Unfortunately, no further analyses of the relationship between HT treatment parameters with clinical outcomes in studies using triplet therapy were reported.

Furthermore, the optimal interval between heat, radiation and anticancer drugs is still unclear. With reference to preclinical and clinical outcomes, $t_{i n t}$ affects the thermal enhancement effect of HT on both ionizing radiation and CT drugs. A particular interaction between HT and CT in terms of $t_{\text {int }}$ was reported according to properties of the CT drugs. A short $t_{\text {int }}$ between sequential HT and doxorubicin resulted in more rapid treatment response [153]. However, it is not clear whether the CT drug interacts primarily with RT only when administered on the same day or also during an extended time period. In the first scenario, $\mathrm{CT}$ and HT could typically be administered within a range of 1-6 h prior to RT to optimally exploit the biological interaction. 
Table 11. Prospective clinical studies using RT and CT in combination with HT.

\begin{tabular}{|c|c|c|c|c|c|c|c|c|c|c|}
\hline Author(s) & Cancer Site, $n$ & $\begin{array}{l}\text { CT Drug(s) } \\
\left(\mathrm{mg} / \mathrm{m}^{2}\right) \times \\
\text { Cycles }\end{array}$ & $\begin{array}{l}\text { RT Dose (Gy) } \\
\text { /Fractions }\end{array}$ & $\begin{array}{l}\text { Temperature } \\
\text { Metrics }\left({ }^{\circ} \mathrm{C}\right)\end{array}$ & Session & $\begin{array}{c}t_{\text {treat }} \\
(\mathrm{min})\end{array}$ & $\begin{array}{l}\text { Thermal } \\
\text { Dose (min) }\end{array}$ & $\begin{array}{c}t_{\text {int }} \\
(\min )\end{array}$ & Sequence & $\begin{array}{l}\text { Clinical Outcome } \\
\text { (Comment) }\end{array}$ \\
\hline $\begin{array}{l}\text { Amichetti } \\
\text { et al. [152] }\end{array}$ & $\begin{array}{c}\text { Locally } \\
\text { advanced } \\
\text { head \& neck } \\
\text { cancer, } n=18\end{array}$ & $\begin{array}{l}20 \text { cisplatin } \\
\text { once weekly } \\
\times 7\end{array}$ & $70.0 / 35$ & $\begin{array}{c}\mathrm{T}_{\max }{ }^{\dagger}: 43.2 \\
(41.5-44.5) \\
\mathrm{T}_{\min }{ }^{+}: 40.1 \\
(37-42) \\
\mathrm{T}_{90}{ }^{+}: 40.4 \\
(38.7-42.2)\end{array}$ & $\begin{array}{c}\mathrm{N}_{\text {total }}: \\
2 \\
\mathrm{~N}_{\text {week }}: \\
2\end{array}$ & 30 & $\begin{array}{c}\mathrm{CEM} 42.5^{\circ} \mathrm{C} \\
\mathrm{T}_{\min }{ }^{+}: \\
4.36(0-27) \\
\mathrm{CEM} 42.5^{\circ} \mathrm{C} \\
\mathrm{T}_{\max }{ }^{+}: \\
88(31.8-174)\end{array}$ & 20 & $\begin{array}{l}\text { HT after } \\
\text { RT \& CT }\end{array}$ & $\begin{array}{l}\text { - } \mathrm{CR}^{1}: 72.2 \%(13 / 18), \mathrm{PR}^{2}: \\
16.6 \%(3 / 18) ; \mathrm{NC}^{3}: 11.1 \% \\
\text { - } 2 / 18) . \\
\text { OS }{ }^{4}: 88.8 \%, 3 \text {-year } \\
\text { actuarial survival and } \\
\text { probability of remaining } \\
\text { free of nodal disease: } \\
50.3 \% \text { and } \\
53.3 \%, \text { respectively. } \\
\text { No temperature metrics } \\
\text { correlated with an } \\
\text { increased acute side } \\
\text { effects and the amount of } \\
\text { skin toxicity. }\end{array}$ \\
\hline
\end{tabular}


Table 11. Cont.

\begin{tabular}{|c|c|c|c|c|c|c|c|c|c|c|}
\hline Author(s) & Cancer Site, $n$ & $\begin{array}{l}\text { CT Drug(s) } \\
\left(\mathrm{mg} / \mathrm{m}^{2}\right) \times \\
\text { Cycles }\end{array}$ & $\begin{array}{l}\text { RT Dose (Gy) } \\
\text { /Fractions }\end{array}$ & $\begin{array}{l}\text { Temperature } \\
\text { Metrics }\left({ }^{\circ} \mathrm{C}\right)\end{array}$ & Session & $\begin{array}{l}t_{\text {treat }} \\
(\min )\end{array}$ & $\begin{array}{l}\text { Thermal } \\
\text { Dose (min) }\end{array}$ & $\begin{array}{c}t_{\text {int }} \\
(\mathrm{min})\end{array}$ & Sequence & $\begin{array}{l}\text { Clinical Outcome } \\
\text { (Comment) }\end{array}$ \\
\hline Asao et al. [151] & $\begin{array}{c}\text { Locally } \\
\text { advanced } \\
\text { rectal } \\
\text { cancer, } \\
n=29\end{array}$ & $\begin{array}{c}250 \\
5- \\
\text { fluorouracil } \\
\text { for } 5 \text { days } \\
\times 2 \\
25 \\
\text { for } 5 \text { days } \\
\times 2\end{array}$ & $\begin{array}{l}40.0-50.0 \\
/ 20-25\end{array}$ & $\begin{array}{c}\mathrm{T}_{\max }{ }^{\dagger}: \\
40.3 \pm 0.89 \\
(38.6-41.9)\end{array}$ & $\begin{array}{c}\mathrm{N}_{\text {total }}: \\
3 \\
\mathrm{~N}_{\text {week }}: \\
1\end{array}$ & 60 & n.r. & n.r. & $\begin{array}{l}\text { HT after RT } \\
\text { during } \mathrm{CT}\end{array}$ & $\begin{array}{l}\text { - Toxicity, grade III: } 2 \\
\text { patients. } \\
\text { CR: } 55.5 \% \text { in patients with } \\
\text { a total radiation dose of } \\
50 \text { Gy, which was } \\
\text { significantly higher } \\
\text { compared to patients } \\
\text { treated with } 40 \mathrm{~Gy} . \\
\text { - } 41.4 \% \text { of patients had } \\
\text { significant downstaging. } \\
\text { (correlation of thermometric } \\
\text { parameters with clinical } \\
\text { outcome not reported) }\end{array}$ \\
\hline $\begin{array}{l}\text { Westermann } \\
\text { et al. [150] }\end{array}$ & $\begin{array}{l}\text { Cervix } \\
\text { cancer, } \\
n=68\end{array}$ & $\begin{array}{l}40 \text { cisplatin } \\
\text { once weekly } \\
\quad \times 35\end{array}$ & $\begin{array}{c}45.0-50.4 \\
/ 25-28 \\
\text { with } \\
\text { LDR- IRT }^{7} \\
\text { and } \\
\text { HDR-IRT }^{7} \\
\quad\left({ }^{192} \mathrm{Ir}\right)\end{array}$ & $\begin{array}{l}\mathrm{T}_{90}{ }^{+}: 39.4 \\
\mathrm{~T}_{50}{ }^{+}: 40.7\end{array}$ & $\begin{array}{c}\mathrm{N}_{\text {total }}: \\
8-10 \\
\mathrm{~N}_{\text {week }}: \\
1\end{array}$ & 60 & n.r. & n.r. & $\begin{array}{c}\text { HT \& CT } \\
\text { after/before } \\
\text { RT }\end{array}$ & $\begin{array}{l}\text { CR: } 90 \%, 2-y e a r \text { DFS } 5 \\
\text { and OS: } 71.6 \% \text { and } \\
78.5 \% \text {, respectively. } \\
\text { - } \quad \text { A significant difference in } \\
\text { DFS between Netherlands } \\
\text { and US clinical centers. } \\
\text { - Specific toxicity } \\
\text { associated with HT } \\
\text { was mild. } \\
\text { (correlation of thermometric } \\
\text { parameters with clinical } \\
\text { outcome not reported) }\end{array}$ \\
\hline
\end{tabular}


Table 11. Cont.

\begin{tabular}{|c|c|c|c|c|c|c|c|c|c|c|}
\hline Author(s) & Cancer Site, $n$ & $\begin{array}{l}\text { CT Drug(s) } \\
\left(\mathrm{mg} / \mathrm{m}^{2}\right) \times \\
\text { Cycles }\end{array}$ & $\begin{array}{l}\text { RT Dose (Gy) } \\
\text { /Fractions }\end{array}$ & $\begin{array}{l}\text { Temperature } \\
\text { Metrics }\left({ }^{\circ} \mathrm{C}\right)\end{array}$ & Session & $\begin{array}{c}t_{\text {treat }} \\
\text { (min) }\end{array}$ & $\begin{array}{c}\text { Thermal } \\
\text { Dose (min) }\end{array}$ & $\begin{array}{c}t_{\text {int }} \\
(\mathrm{min})\end{array}$ & Sequence & $\begin{array}{l}\text { Clinical Outcome } \\
\text { (Comment) }\end{array}$ \\
\hline Harima et al. [149] & $\begin{array}{c}\text { Locally } \\
\text { advanced } \\
\text { cervical } \\
\text { cancer, } n=51\end{array}$ & $\begin{array}{c}30-40 \\
\text { cisplatin } \\
\text { once weekly } \\
\times 3-5\end{array}$ & $\begin{array}{c}30.0-50.0 \\
\text { /15-25 } \\
\text { with } \\
\text { LDR- IRT7 } \\
\text { (192 Ir): } \\
5.0-6.0 \\
\text { /3-5 }\end{array}$ & $\begin{array}{c}\mathrm{T}_{\max }{ }^{\dagger}: 42.2 \\
(40.1-44.6) \\
\mathrm{T}_{\mathrm{avg}}{ }^{\dagger}: 41.1 \\
(39.6-42.5) \\
\text { Data from } \\
\text { Ohguri et al. } \\
{[155]} \\
\mathrm{T}_{90}{ }^{\ddagger} 38.9 \\
(37.7-42.2) \\
\mathrm{T}_{50} \ddagger: 39.9 \\
(38.4-42.4)\end{array}$ & $\begin{array}{c}\mathrm{N}_{\text {total }}: \\
4-6 \\
\mathrm{~N}_{\text {week }}: \\
1\end{array}$ & 60 & $\begin{array}{c}\mathrm{CEM} 43^{\circ} \mathrm{CT}_{90} \\
{ }^{\circ}: \\
3.8(0.1-46.6)\end{array}$ & 20 & $\begin{array}{l}\text { HT after } \\
\text { RT\&CT }\end{array}$ & $\begin{array}{ll}\text { - } & \text { CR: } 88 \%(44 / 50) . \\
\text { - } & \text { 5-year OS, DFS, and LPFS } \\
& 6 \text { were } 77.8 \%, 70.8 \% \text { and } \\
& 80.1 \% \text {, respectively. } \\
\text { - } \quad \text { It was well tolerated and } \\
\text { caused no additional } \\
\text { acute or long } \\
\text { term toxicity. } \\
\text { - Ohguri et al. [155]: } \\
\quad \text { CEM43 }{ }^{\circ} \mathrm{CT}_{90} \geq 1 \text { min } \\
\text { tended to predict better } \\
\text { DFS and CR. }\end{array}$ \\
\hline $\begin{array}{l}\text { Kouloulias } \\
\text { et al. [153] }\end{array}$ & $\begin{array}{c}\text { Recurrent } \\
\text { breast } \\
\text { cancer, } \\
n=15\end{array}$ & $\begin{array}{c}40-60 \\
\text { liposomal } \\
\text { doxorubicin } \\
\text { once } \\
\text { monthly } \\
\times 6\end{array}$ & $30.6 / 17$ & $\begin{array}{c}\mathrm{T}_{\max }{ }^{\dagger}: 43.2 \\
(41.5-44.5) \\
\mathrm{T}_{\min }{ }^{+}: 45.0 \\
(44.2-45.7)\end{array}$ & $\begin{array}{l}\mathrm{N}_{\text {total }}: \\
\quad 6 \\
\mathrm{~N}_{\text {monthly }}: \\
1\end{array}$ & 60 & n.r. & $180-240$ & $\begin{array}{l}\text { HT after } \\
\text { CT\&RT }\end{array}$ & $\begin{array}{l}\text { - CR: } 2 \%(3 / 15), \text { PR: } 80 \% \\
\text { - } 12 / 15) ; \\
\text { CR or PR obtained more } \\
\text { quickly with a shorter } t_{\text {int }} \\
\text { between HT and CT. } \\
\text { DFILR }{ }^{7} \text { was better for } \\
\mathrm{T}_{90}>44^{\circ} \mathrm{C} \text { of } \geq 16 \text { min } \\
\text { compared with those for } \\
\text { whom } \mathrm{T}_{90}>44^{\circ} \mathrm{C} \text { of } \\
<16 \text { min. } \\
\text { DFILR was significantly } \\
\text { correlated with } \mathrm{T}_{\text {min }}{ }^{\dagger} \text { but } \\
\text { not with } \mathrm{T}_{\max }{ }^{+} .\end{array}$ \\
\hline
\end{tabular}


Table 11. Cont.

\begin{tabular}{|c|c|c|c|c|c|c|c|c|c|c|}
\hline Author(s) & Cancer Site, $n$ & $\begin{array}{l}\text { CT Drug(s) } \\
\left(\mathrm{mg} / \mathrm{m}^{2}\right) \times \\
\text { Cycles }\end{array}$ & $\begin{array}{l}\text { RT Dose (Gy) } \\
\text { /Fractions }\end{array}$ & $\begin{array}{l}\text { Temperature } \\
\text { Metrics }\left({ }^{\circ} \mathrm{C}\right)\end{array}$ & Session & $\begin{array}{l}t_{\text {treat }} \\
\text { (min) }\end{array}$ & $\begin{array}{l}\text { Thermal } \\
\text { Dose (min) }\end{array}$ & $\begin{array}{c}t_{\text {int }} \\
(\mathrm{min})\end{array}$ & Sequence & $\begin{array}{l}\text { Clinical Outcome } \\
\text { (Comment) }\end{array}$ \\
\hline $\begin{array}{l}\text { Herman } \\
\text { et al. [146] }\end{array}$ & $\begin{array}{c}\text { Locally } \\
\text { advanced } \\
\text { malignancies, } \\
n=24\end{array}$ & $\begin{array}{c}20-50 \\
\text { cisplatin } \\
\text { once weekly } \\
\times 6\end{array}$ & $\begin{array}{c}60.0-66.0 \\
/ 30-33 \\
\text { or } \\
24.0-36.0 \\
/ 12-18\end{array}$ & $\begin{array}{c}\mathrm{T}_{\max }{ }^{\dagger}: \\
43.7 \pm 2.6 \\
\mathrm{~T}_{\min }{ }^{+}: \\
38.2 \pm 2.0 \\
\mathrm{~T}_{\text {avg }}{ }^{+}: \\
40.8 \pm 1.9\end{array}$ & $\begin{array}{c}\mathrm{N}_{\text {total }}: \\
6 \\
\mathrm{~N}_{\text {week }}: \\
1\end{array}$ & 60 & $\begin{array}{c}\mathrm{CEM} 42{ }^{\circ} \mathrm{CT}_{90} \\
{ }^{\circ}: \\
11.2 \pm 21.3 \\
\mathrm{CEM}^{\circ} 3^{\circ} \mathrm{CT}_{90} \\
{ }^{+}: \\
3.1 \pm 5.4\end{array}$ & n.r. & $\begin{array}{l}\text { HT before } \\
\text { CT\&RT }\end{array}$ & $\begin{array}{l}\text { CR: } 50 \%(12 / 24), \text { PR: } 50 \% \\
\text { (12/24); } \\
\text { No grade IIII } \\
\text { acute toxicity. } \\
\text { Late toxicity, grade IV: } \\
\text { only } 1 \text { patient. } \\
\text { - With thermal dose of } \\
\mathrm{CEM}^{\circ} 3^{\circ} \mathrm{CT}_{90}^{+}=4.6 \mathrm{~min}, \\
50 \% \text { of patients achieved } \\
\mathrm{CR} \text { and with } \\
\mathrm{CEM} 43^{\circ} \mathrm{CT}_{90}^{+}=2.0 \mathrm{~min}, \\
50 \% \text { patients achieved PR. } \\
\text { Cisplatin concentration } \\
\text { amount correlated } \\
\text { with CR. }\end{array}$ \\
\hline
\end{tabular}


Table 11. Cont.

\begin{tabular}{|c|c|c|c|c|c|c|c|c|c|c|}
\hline Author(s) & Cancer Site, $n$ & $\begin{array}{l}\text { CT Drug(s) } \\
\left(\mathrm{mg} / \mathrm{m}^{2}\right) \times \\
\text { Cycles }\end{array}$ & $\begin{array}{l}\text { RT Dose (Gy) } \\
\text { /Fractions }\end{array}$ & $\begin{array}{l}\text { Temperature } \\
\text { Metrics }\left({ }^{\circ} \mathrm{C}\right)\end{array}$ & Session & $\begin{array}{c}\mathbf{t}_{\text {treat }} \\
(\mathrm{min})\end{array}$ & $\begin{array}{c}\text { Thermal } \\
\text { Dose (min) }\end{array}$ & $\begin{array}{c}\mathbf{t}_{\text {int }} \\
(\mathrm{min})\end{array}$ & Sequence & $\begin{array}{l}\text { Clinical Outcome } \\
\text { (Comment) }\end{array}$ \\
\hline Ott et al. [158] & $\begin{array}{c}\text { Locally } \\
\text { advanced } \\
\text { or recurrent } \\
\text { rectal } \\
\text { cancer, } \\
n=105\end{array}$ & $\begin{array}{c}250 \\
5- \\
\text { fluorouracil } \\
\text { on days } 1-14 \\
\text { and } 22-35 \\
\text { or } \\
1650 \\
\text { capecitabine } \\
\text { on days 1-14 } \\
\text { and 22-35 } \\
50 \\
\text { oxaliplatin } \\
\times 4\end{array}$ & $\begin{array}{c}\text { LARC } \\
50.4 / 28 \\
\text { LCC } \\
45 / 25\end{array}$ & n.r. & $\begin{array}{c}\mathrm{N}_{\text {total }} \\
\quad \neq: \\
10 \\
\mathrm{~N}_{\text {week }} \text { : } \\
2\end{array}$ & 60 & $\begin{array}{l}\text { LARC }^{19} \\
\mathrm{CEM}^{\circ} \mathrm{C}^{\dagger}: \\
6.4 \pm 5.2 \\
\mathrm{LCC}^{20} \\
\mathrm{CEM}^{\circ} 3^{\circ} \mathrm{C}^{\dagger}: \\
6.4 \pm 4.9\end{array}$ & n.r. & HT before RT & $\begin{array}{l}\text { - } 11 \%(2 / 19) \text { and } 27 \% \\
(16 / 59) \mathrm{DLT}^{8} \text { criteria, } \\
\text { corresponding to FR }{ }^{9} \text { : } \\
90 \% \text { and } 73 \% \text {, } \\
\text { respectively. } \\
\text { Pathological CR: } 20 \% \\
(19 / 95), \mathrm{CTR}^{10}: 28 \% \\
(18 / 64) \text { and } 38 \%(3 / 8) \text { in } \\
\text { patients with LARC and } \\
\text { LRRC, respectively. } \\
\text { 5-year OS: } 75 \% \text { for the } \\
\text { whole group. } \\
\text { No grade } \\
\text { 4-5 adverse events. } \\
\text { (correlation of thermometric } \\
\text { parameters with clinical } \\
\text { outcome not presented) }\end{array}$ \\
\hline Gani et al. [154] & $\begin{array}{l}\text { Locally } \\
\text { advanced } \\
\text { rectal } \\
\text { cancer, } \\
n=78\end{array}$ & $\begin{array}{c}1000 \\
5- \\
\text { fluorouracil } \\
\times 4\end{array}$ & $50.4 / 28$ & $\begin{array}{c}\mathrm{T}_{90} \neq: 39.5 \\
\text { (IQR: } \\
39.1-39.9)\end{array}$ & $\begin{array}{l}\mathrm{N}_{\text {total }}: \\
8 \\
\mathrm{~N}_{\text {week }}: \\
2\end{array}$ & 60 & $\begin{array}{c}\text { CEM } 43^{\circ} \mathrm{C} \ddagger: \\
4.5 \\
\text { (IQR: } 2.2-8.2)\end{array}$ & n.r. & n.r. & 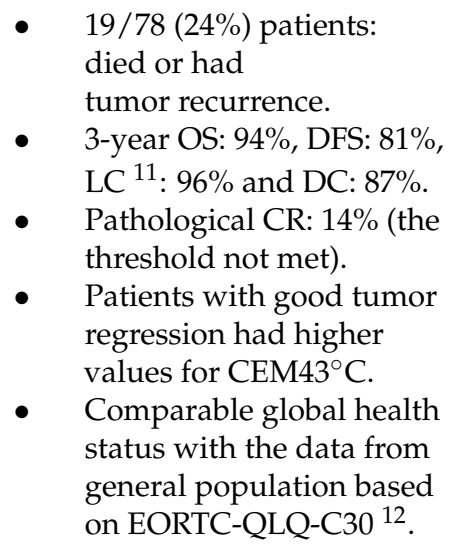 \\
\hline
\end{tabular}


Table 11. Cont.

\begin{tabular}{|c|c|c|c|c|c|c|c|c|c|c|}
\hline Author(s) & Cancer Site, $n$ & $\begin{array}{c}\text { CT Drug(s) } \\
\left(\mathrm{mg} / \mathrm{m}^{2}\right) \times \\
\text { Cycles }\end{array}$ & $\begin{array}{c}\text { RT Dose (Gy) } \\
\text { /Fractions }\end{array}$ & $\begin{array}{l}\text { Temperature } \\
\text { Metrics }\left({ }^{\circ} \mathrm{C}\right)\end{array}$ & Session & $\begin{array}{l}t_{\text {treat }} \\
(\min )\end{array}$ & $\begin{array}{l}\text { Thermal } \\
\text { Dose (min) }\end{array}$ & $\begin{array}{c}t_{\text {int }} \\
(\min )\end{array}$ & Sequence & $\begin{array}{l}\text { Clinical Outcome } \\
\text { (Comment) }\end{array}$ \\
\hline Rau et al. [159] & $\begin{array}{l}\text { Locally } \\
\text { advanced } \\
\text { rectal } \\
\text { cancer, } \\
n=37\end{array}$ & $\begin{array}{l}300-350 \\
5- \\
\text { fluorouracil } \\
50 * \mathrm{mg} \\
\text { leucovorin } \\
5 \text { times } \\
\text { weekly } \\
\times 2\end{array}$ & $\begin{array}{c}45.0-50.0 \\
\quad / 25\end{array}$ & $\begin{array}{c}\text { Data from Rau } \\
\text { et al. [160]: } \\
\mathrm{T}_{90}{ }^{+}: 40.2 \pm \\
1.2 \\
\mathrm{~T}_{\max }{ }^{+}: 41.4 \pm \\
0.6\end{array}$ & $\begin{array}{c}\mathrm{N}_{\text {total }} \\
\neq: \\
5 \\
\mathrm{~N}_{\text {week }}: \\
1\end{array}$ & 60 & 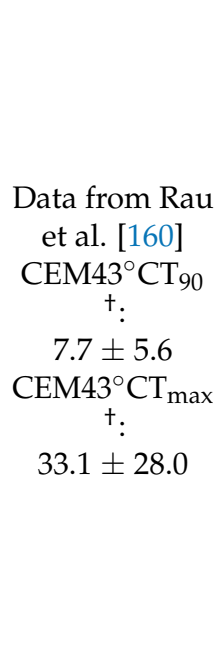 & n.r. & $\begin{array}{c}\text { RT after } \\
\text { concurrent } \\
\text { HT\&CT }\end{array}$ & $\begin{array}{ll}\text { - } & \text { Grade III toxicity: } 16 \% . \\
\text { ORR } 13: 89 \% \text {, and } 31 \\
\text { resection specimens had } \\
\text { negative margins. } \\
\text { RR } 14: 59.4 \%, \text { CR: } 14 \%, \\
\text { OS: } 56 \% \text {. } \\
\text { Cumulative minutes at } \\
\mathrm{T}_{90} \geq 40.5^{\circ} \mathrm{C} \text { and } \mathrm{T}_{90} \\
\text { correlate with the RR but } \\
\text { not with long term OS } \\
\text { and DFSR }{ }^{15} \text { [160] but } \\
\mathrm{T}_{\text {max }} \text { showed no } \\
\text { significant influence } \\
\text { on RR. } \\
\text { RR: } 33 \% \text { when } \\
\text { T } \mathrm{T}_{90}<40.5^{\circ} \mathrm{C} \text { and } \mathrm{RR}: 75 \% \\
\text { response, } \mathrm{T}_{90}>40.5{ }^{\circ} \mathrm{C} .\end{array}$ \\
\hline $\begin{array}{l}\text { Wittlinger } \\
\text { et al. [161] }\end{array}$ & $\begin{array}{c}\text { Bladder } \\
\text { cancer, } \\
n=45\end{array}$ & $\begin{array}{c}20 \text { cisplatin } \\
5 \text { times } \\
\text { weekly } \\
\times 2 \\
600 \\
5- \\
\text { fluorouracil } \\
5 \text { times } \\
\text { weekly } \\
\times 2\end{array}$ & $\begin{array}{c}50.4-55.8 / \\
28-31\end{array}$ & $\begin{array}{c}\mathrm{T}_{\text {avg }}{ }^{\dagger}: 40.8 \\
\text { (95\%CI: } \\
40.5-41.6)\end{array}$ & $\begin{array}{c}\mathrm{N}_{\text {total }}: \\
5-7 \\
\mathrm{~N}_{\text {week }}: \\
1\end{array}$ & 60 & $\begin{array}{c}\mathrm{CEM}^{\circ} 3^{\circ} \mathrm{C}^{\dagger}: 57 \\
(95 \% \mathrm{CI}: \\
40.5-41.6)\end{array}$ & 60 & $\begin{array}{l}\text { RT after } \\
\text { concurrent } \\
\text { CT\&HT }\end{array}$ & $\begin{array}{l}\text { - } \quad \text { CR: } 96 \%, \text { NC: } 4 \% \text {. } \\
\text { Freedom from any local } \\
\text { and distant relapse: } 69 \% \\
\text { and relapse: } 16 \% \text {. } \\
\text { 3-year bladder } \\
\text { preservation: } 96 \%, \text { LPFS: } \\
\text { 81\%, DSS: } 88 \% \text {, DFS: } 71 \% \text {, } \\
\text { OS: } 80 \% \text { and MFS }{ }^{16}: 89 \% \text {. } \\
\text { - One of significant } \\
\text { prognostic factors for } \\
\text { OS: } \mathrm{N}_{\text {week }} \\
\text { - Acute toxicity, grades } \\
\text { III-IV: } 27 \% \text {. } \\
\text { Late toxicity, grades } \\
\text { III-IV: } 24 \% .\end{array}$ \\
\hline
\end{tabular}


Table 11. Cont.

\begin{tabular}{|c|c|c|c|c|c|c|c|c|c|c|}
\hline Author(s) & Cancer Site, $n$ & $\begin{array}{c}\text { CT Drug(s) } \\
\left(\mathrm{mg} / \mathrm{m}^{2}\right) \times \\
\text { Cycles }\end{array}$ & $\begin{array}{l}\text { RT Dose (Gy) } \\
\text { /Fractions }\end{array}$ & $\begin{array}{l}\text { Temperature } \\
\text { Metrics }\left({ }^{\circ} \mathrm{C}\right)\end{array}$ & Session & $\begin{array}{l}t_{\text {treat }} \\
(\mathrm{min})\end{array}$ & $\begin{array}{c}\text { Thermal } \\
\text { Dose (min) }\end{array}$ & $\begin{array}{c}t_{\text {int }} \\
(\mathrm{min})\end{array}$ & Sequence & $\begin{array}{l}\text { Clinical Outcome } \\
\text { (Comment) }\end{array}$ \\
\hline Milani et al. [162] & $\begin{array}{l}\text { Recurrent } \\
\text { rectal } \\
\text { cancer, } \\
n=24\end{array}$ & $\begin{array}{c}350 \\
5- \\
\text { fluorouracil } \\
5 \text { times } \\
\text { weekly } \\
\times 4 \\
\text { (continuous } \\
\text { infusion) }\end{array}$ & $\begin{array}{l}30.0-45.0 / \\
16-25\end{array}$ & $\begin{array}{l}\mathrm{T}_{90}{ }^{+}: 41.4 \\
\mathrm{~T}_{50}{ }^{+}: 42.9 \\
\mathrm{~T}_{20}{ }^{\dagger}: 43.5\end{array}$ & $\begin{array}{c}\mathrm{N}_{\text {total }} \\
\neq: \\
8 \\
\mathrm{~N}_{\text {week }}: \\
2 \\
\end{array}$ & 60 & n.r. & 60 & $\begin{array}{l}\text { HT after } \\
\text { concurrent } \\
\text { RT\&CT }\end{array}$ & $\begin{array}{l}\text { CR: 0\% (0/20), PR: 10\% } \\
\text { (2/20), NC: 85\% (17/20), } \\
\text { PD: 5\% (1/20). } \\
\text { 1-year OS, DMFS } 17, \\
\text { LPFR } 18: 87 \%, 82 \%, 61 \% \text {, } \\
\text { respectively. } \\
\text { 2-year OS, DMFS, LPFR: } \\
\text { 60\%, 52\%, 30\%, } \\
\text { respectively. } \\
\text { 3-year OS, DMFS, LPFR: } \\
\text { 30\%, 39\%, 15\%, } \\
\text { respectively. } \\
\text { Acute toxicity, grade III: } \\
\text { 12.5\% of the patients. } \\
\text { (correlation of thermometric } \\
\text { parameters with clinical } \\
\text { outcome not presented) }\end{array}$ \\
\hline
\end{tabular}

$n$ : number of patients assigned to be treated with $\mathrm{HT}$ in combination with RT and $\mathrm{CT} ;{ }^{+}:$mean value ( \pm standard deviation) or mean value (range); ${ }^{\ddagger}:$ median (range); ${ }^{1} \mathrm{CR}:$ complete response; ${ }^{2}$ PR: partial response; ${ }^{3}$ NC: no change; ${ }^{4}$ OS: overall survival, ${ }^{5}$ DFS: disease free survival; ${ }^{6}$ LPFS: local progression free survival; ${ }^{7}$ DFILR: disease-free interval to local relapse; ${ }^{8}$ DLT: dose limiting toxicities; ${ }^{9}$ FR: feasibility rate; ${ }^{10}$ CTR: complete tumor regression; ${ }^{11}$ LC: local control; ${ }^{12}$ EORTC-QLQ: European Organization for research and treatment of cancer-quality of life questionnaire; ${ }^{13}$ ORR: objective response rate; ${ }^{14}$ RR: response rate; ${ }^{15}$ DFSR: disease-free survival rate; ${ }^{16}$ MFS: metastasis-free survival; ${ }^{17}$ DMFS: distant metastases-free survival; ${ }^{18}$ LPFR: local progression-free survival; ${ }^{19}$ LARC: locally advanced rectal cancer; ${ }^{20}$ LCC: recurrent rectal cancer. 
Table 12. Retrospective clinical studies using RT and CT in combination with HT.

\begin{tabular}{|c|c|c|c|c|c|c|c|c|c|c|}
\hline Author(s) & Cancer Site, $n$ & $\begin{array}{c}\text { CT Drug } \\
(\mathrm{s})\left(\mathrm{mg} / \mathrm{m}^{2}\right) \\
\text { Cycles }\end{array}$ & $\begin{array}{l}\text { RT Dose (Gy) } \\
\text { /Fractions }\end{array}$ & $\begin{array}{l}\text { Temperature } \\
\text { Metrics }\left({ }^{\circ} \mathrm{C}\right)\end{array}$ & Session & $\begin{array}{l}t_{\text {treat }} \\
\text { (min) }\end{array}$ & $\begin{array}{l}\text { Thermal } \\
\text { Dose (min) }\end{array}$ & $\begin{array}{c}t_{\text {int }} \\
(\min )\end{array}$ & Sequence & $\begin{array}{l}\text { Clinical Outcome } \\
\text { (Comment) }\end{array}$ \\
\hline Zhu et al. [163] & $\begin{array}{l}\text { Locally } \\
\text { advanced } \\
\text { esophageal } \\
\text { cancer, } \\
n=78\end{array}$ & $\begin{array}{c}450 \\
\text { 5-fluorouracil } \\
\text { five times } \\
\text { weekly } \\
\times 4-6 \\
25 \text { cisplatin } \\
\text { five times } \\
\text { weekly } \\
\times 4-6 \\
6\end{array}$ & $\begin{array}{c}60.0-66.0 \\
/ 30-33\end{array}$ & n.r. & $\begin{array}{c}\mathrm{N}_{\text {total }}: \\
6-12 \\
\mathrm{~N}_{\text {week }}: \\
2\end{array}$ & 60 & n.r. & 120 & n.r. & 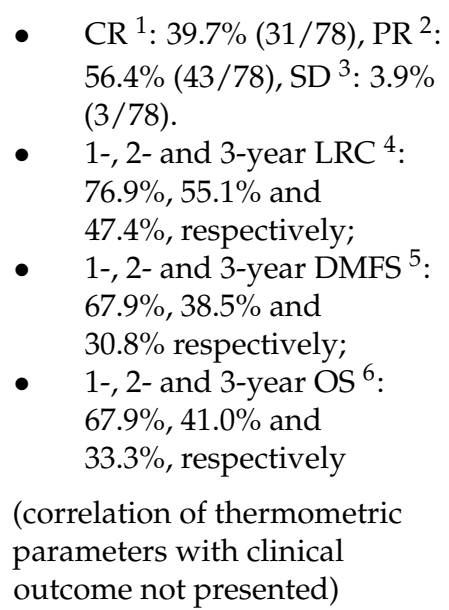 \\
\hline
\end{tabular}


Table 12. Cont.

\begin{tabular}{|c|c|c|c|c|c|c|c|c|c|c|}
\hline Author(s) & Cancer Site, $n$ & $\begin{array}{c}\text { CT Drug } \\
(\mathrm{s})\left(\mathrm{mg} / \mathrm{m}^{2}\right) \\
\text { Cycles }\end{array}$ & $\begin{array}{c}\text { RT Dose (Gy) } \\
\text { /Fractions }\end{array}$ & $\begin{array}{l}\text { Temperature } \\
\text { Metrics }\left({ }^{\circ} \mathrm{C}\right)\end{array}$ & Session & $\begin{array}{l}\mathbf{t}_{\text {treat }} \\
(\mathrm{min})\end{array}$ & $\begin{array}{l}\text { Thermal } \\
\text { Dose (min) }\end{array}$ & $\begin{array}{c}t_{\text {int }} \\
(\mathrm{min})\end{array}$ & Sequence & $\begin{array}{l}\text { Clinical Outcome } \\
\text { (Comment) }\end{array}$ \\
\hline Gani et al. [164] & $\begin{array}{l}\text { Locally } \\
\text { advanced } \\
\text { rectal } \\
\text { cancer, } \\
n=60\end{array}$ & $\begin{array}{c}1000 \\
\text { 5-fluorouracil } \\
\times 4\end{array}$ & $50.4 / 28$ & $\begin{array}{l}\mathrm{T}_{90} \ddagger: 39.3 \\
(37.1-40.6)\end{array}$ & $\begin{array}{c}\mathrm{N}_{\text {total }}{ }^{\ddagger}: \\
4 \\
\mathrm{~N}_{\text {week }}: \\
1-2\end{array}$ & 60 & $\begin{array}{l}\text { CEM } 43^{\circ} \mathrm{C} \ddagger: \\
1.1(0.0-9.2)\end{array}$ & n.r. & n.r. & $\begin{array}{l}\text { - } 5 \text {-year OS, DFS }{ }^{11} \text {, local } \\
\text { control and DMFS were } \\
83 \%, 75 \%, 93 \% \text { and } \\
76 \% \text {, respectively. } \\
\text { - } \quad \text { o impact of HT on DFS } \\
\text { and DMFS. } \\
\quad \mathrm{N}_{\text {total }} \text { not predictive for } \\
\quad \text { OS, DFS, LC, or DMFS. } \\
\text { Postoperative nodal stage } \\
\text { remained a significant } \\
\text { prognosticator for OS, DFS and } \\
\text { DMFS (multivariate analysis). }\end{array}$ \\
\hline Merten et al. [165] & $\begin{array}{c}\text { Bladder } \\
\text { cancer, } \\
n=79\end{array}$ & $\begin{array}{c}20 \text { cisplatin } \\
5 \text { times weekly } \\
\times 2 \\
600 \\
5 \text {-fluorouracil } \\
5 \text { times weekly } \\
\times 2\end{array}$ & $\begin{array}{c}50.4-55.8 / \\
28-31\end{array}$ & n.r. & $\begin{array}{c}\mathrm{N}_{\text {total }}: \\
5-7 \\
\mathrm{~N}_{\text {week }}: \\
1\end{array}$ & 60 & n.r. & $0-60$ & $\begin{array}{l}\text { RT after } \\
\text { concurrent } \\
\text { CT\&HT }\end{array}$ & $\begin{array}{ll}\text { - } & \text { CR: } 87 \% \text { (67/77). } \\
\text { - } & \text { 5- and 10-year OS: } 87 \% \\
& \text { and 60\%, respectively. } \\
\text { - } & \text { 5- and 10-year DFS to } 66 \% \\
& \text { and } 46 \% \text { respectively. } \\
\text { - } & \text { Acute toxicity, grade III: } \\
& 11 \% \text { and grade IV: } 3 \% . \\
\text { - } \quad \text { Late toxicity, grade } \\
\quad \text { III: } 1.3 \% \text {. } \\
\text { (correlation of thermometric } \\
\text { parameters with clinical } \\
\text { outcome not presented) }\end{array}$ \\
\hline
\end{tabular}


Table 12. Cont.

\begin{tabular}{|c|c|c|c|c|c|c|c|c|c|c|}
\hline Author(s) & Cancer Site, $n$ & $\begin{array}{c}\text { CT Drug } \\
(\mathrm{s})\left(\mathrm{mg} / \mathrm{m}^{2}\right) \times \\
\text { Cycles }\end{array}$ & $\begin{array}{l}\text { RT Dose (Gy) } \\
\text { /Fractions }\end{array}$ & $\begin{array}{l}\text { Temperature } \\
\text { Metrics }\left({ }^{\circ} \mathrm{C}\right)\end{array}$ & Session & $\begin{array}{l}t_{\text {treat }} \\
(\min )\end{array}$ & $\begin{array}{c}\text { Thermal } \\
\text { Dose (min) }\end{array}$ & $\begin{array}{c}t_{\text {int }} \\
(\mathrm{min})\end{array}$ & Sequence & $\begin{array}{l}\text { Clinical Outcome } \\
\text { (Comment) }\end{array}$ \\
\hline $\begin{array}{l}\text { van Haaren } \\
\text { et al. [166] }\end{array}$ & $\begin{array}{l}\text { Esophageal } \\
\text { cancer, } \\
n=29\end{array}$ & $\begin{array}{c}50 \\
\text { paclitaxelonce } \\
\text { weekly } \\
\times 5 \\
\text { and } \\
\text { carboplatin } \\
\text { (AUC }=2) \\
\text { once weekly } \\
\times 5\end{array}$ & $41.4 / 23$ & $\begin{array}{c}\mathrm{T}_{90}{ }^{+}: 38.6 \pm \\
0.5 \\
\mathrm{~T}_{50}{ }^{+}: 39.2 \pm \\
0.6 \\
\mathrm{~T}_{10}{ }^{+}: 40.1 \pm \\
0.8\end{array}$ & $\begin{array}{c}\mathrm{N}_{\text {total }}: \\
5 \\
\mathrm{~N}_{\text {week }}: \\
1\end{array}$ & 60 & n.r. & $0-60$ & $\begin{array}{l}\text { HT after } \\
\text { CT \& RT }\end{array}$ & $\begin{array}{l}\text { CR: } 19 \%(5 / 29), \mathrm{mPR}^{12} \text { : } \\
\text { 26\% }(7 / 29), \mathrm{PR}: 33 \% \\
(9 / 29) \text { and } \mathrm{SD}: \\
\text { 22\% }(6 / 29) . \\
\text { The dependence of } \mathrm{T}_{50} \text { on } \\
\text { the body size parameters } \\
\text { was substantial. } \\
\text { (correlation of thermometric } \\
\text { parameters with clinical } \\
\text { outcome not presented) }\end{array}$ \\
\hline
\end{tabular}


Moreover, the $\mathrm{N}_{\text {total }}$ was shown to be a prognostic factor for $\mathrm{OS}$ for bladder cancer patients treated with combined CT, RT, and HT followed by surgery [161]. In contrast, Gani et al. [164] reported that the number of HT sessions was not predictive for OS, DFS, LC, or distant metastasis-free survival. Neither did the sequencing of CT, HT, and RT in clinical reports follow a specific pattern. Preclinical studies are required to better understand the interaction of $\mathrm{CT}$, RT, and heat and how they should be combined in future clinical trials.

\section{Future Prospects}

The main limitations of HT as a cancer treatment in current clinical practice are the need for better standardization of treatment protocols, up-to-date quality assurance guidelines that are widely applicable and dedicated planning systems to generate patient treatment plans. The wide variation of thermometric parameters derived from clinical studies indicate that HT treatment is currently delivered according to individual clinical center guidelines. Consequently, the comparison of clinical study outcomes is substantially hampered by the large degree of variation in treatment parameters. Regarding the data summarized in Tables 6-11, apart from thermal dose and temperature measured during treatment, other thermometric parameters reported often include only $t_{\text {treat }}, t_{\text {int }}$, or $\mathrm{N}_{\text {week }}$.

Monitoring and measuring temperature is one of the main challenges in routine clinical practice and has hindered the clinical expansion of HT. The future of HT in combination with RT and CT requires novel technical developments for the delivery and measurement of homogenous heating of the malignant tissues. Not all studies (Tables 7-12) recorded temperatures in the region of the tumor. The process of inserting temperature probes to monitor and record the HT is considered invasive and uncomfortable, and sometimes the tumor site is inaccessible for the temperature probe. For example, Milani et al. [162] reported that even though the tumors were not deep-seated, intratumoral temperature measurements were only feasible in one of 24 patients, so no representative thermal doses could be reported. One of the non-invasive approaches currently under clinical evaluation is magnetic resonance thermometry (MRT) that provides 3-D temperature measurements. Hybrid MR/HT devices are currently installed in five European clinical centers.

Temperature measurements in anthropomorphic phantoms with MRT are accurate in comparison with thermistor probes [167], but clinical measurements are currently inaccurate in most pelvic and abdominal tumors [168]. The physiological changes in tissue microenvironment, patient movements, magnetic field drift over time, limited sensitivity in fatty tissues, and respiratory motion, including cardiac activity in regions of the pelvis and abdomen, hamper the accurate temperature measurement by MRT [168]. The temperature images from MRT systems contain image distortion, artifacts, and noise, leading to inaccurate temperature measurement, low temporal resolution, and low imaging to signal-to-noise ratio (SNR) [169]. The sources and solutions of image artifacts as a result of additional frequencies were described by Gellermann et al. [170]. Proton-resonance frequency shift (PRFS), apparent diffusion coefficient (ADC), longitudinal relaxation time $\left(\mathrm{T}_{1}\right)$, transversal relaxation time (T2), and equilibrium magnetization (M0) are the imaging techniques used to exploit temperature-dependent parameters [170-173]. The PRFS technique is the most frequently used MRT method, even though it was shown that when there is a poor magnetic field homogeneity, $\mathrm{ADC}$ or $\mathrm{T}_{1}$ techniques are preferable [174]. However, the accuracy of temperature measurements was in the range of \pm 0.4 to $\pm 0.5{ }^{\circ} \mathrm{C}$ between PRFS method and thermistor probe using a heterogeneous phantom [175]. A stronger correlation between MRT and thermistor probes was found in patients with soft tissue sarcomas of lower extremities and pelvis [176] in comparison with recurrent rectal carcinoma [177]. The successful implementation of MRT in clinical centers, as automated temperature feedback during the HT session, might have a considerable impact on clinical outcomes to deliver the desired heating and conform the heat distribution to spare healthy surrounding tissues. This could substantially help to standardize data collection and the analysis of thermometric parameters. Another experimental approach to monitoring treatment temperature during HT sessions is electrical impedance tomography (EIT) as 
recently reported in a simulation study by Poni et al. [178]. EIT captures the electrical conductivity of tissues depends on temperature elevation. For example, the multifrequency EIT technique detects the changes in conductivity due to perfusion increase induced by the change in temperature [179]. The accuracy of EIT for temperature measurements was reported to range from $1.5^{\circ} \mathrm{C}$ to $5{ }^{\circ} \mathrm{C}$ [180]. The potential of EIT to monitor temperature in the cardiac thermal ablation field is being investigated [181]. This technique also holds promise for HT treatment. Both MRT and EIT may allow for improvement of the spatial homogeneity of heat to the cancer tissues.

The technological advances and standardization of international treatment protocols for different cancer types will improve the effectiveness and synergy of HT in combination with RT and/or CT. In line with this, there is a need for clinically accepted processes for the recording and reporting of thermometric data. This will allow for the inclusion of specific thermometric parameters in future clinical studies combining HT with RT and/or $\mathrm{CT}$. For any future prospective study, it should be mandatory that thermometric parameters are recorded and some recommendations are available in the current guidelines $[43,46]$. The integration of thermometric parameters is one of the objectives of the HYPERBOOST ("Hyperthermia boosting the effect of Radiotherapy") international consortium within the European Horizon 2020 Program MSCA-ITN. The HYPERBOOST network aims to create a novel treatment planning system, including the standardization of thermometric parameters derived from retrospective and prospective clinical trials.

\section{Conclusions}

In this review, we provide an extensive overview of thermometric parameters reported in prospective and retrospective clinical studies which applied HT in combination with $\mathrm{RT}$ and/or CT and their correlation with clinical outcome. It is recognized that there is a wide variety in the practice of HT between clinical centers, and we aimed to elucidate the use and reporting of thermometric parameters in different clinical settings. It emerged that the sequencing of HT and RT varies more than the sequencing of HT and CT. Only a few standards seem to exist with regard to the sequence of HT with RT and CT in a triplet for specific CT drug, RT fractionation and thermal dose. According to the evaluated studies, $t_{\text {int }}$ is a critical parameter in clinical routine, but no clinical reference values have been established. Of note, a constant $t_{\text {treat }}$ of $60 \mathrm{~min}$ throughout the HT treatment course was described in most clinical studies. The most important parameter seems to be temperature itself, which correlates with thermal dose. Revealing the relationship between thermal dose and treatment response for different cancer entities in future clinical studies will lead to the improved application of heat to promote the synergistic actions of HT with RT and CT. We suggest that it become mandatory for new clinical study protocols to include the extensive recording and analysis of thermometric parameters for their validation and overall standardization of HT. This would allow for the definition of thermometric parameters, in particular of thresholds for temperature descriptors and thermal dose.

Author Contributions: Conceptualization, O.R. and P.G.; writing, A.A. and O.R.; writing- review and editing, A.A., O.R., D.P.V., P.G., D.M., H.C., E.O., O.J.O., S.R., P.W., R.A.H., E.P., S.B. and R.F.; visualization, A.A. and O.R.; supervision, H.C., R.F., O.R. and P.G. All authors have read and agreed to the published version of the manuscript.

Funding: This research has received support from the European Union's Horizon 2020 research and innovation programme under the Marie Skłodowska-Curie (MSCA-ITN) grant “Hyperboost" project, no. 955625.

Conflicts of Interest: The authors declare no conflict of interest.

\section{References}

1. Wust, P.; Hildebrandt, B.; Sreenivasa, G.; Rau, B.; Gellermann, J.; Riess, H.; Felix, R.; Schlag, P.M. Hyperthermia in combined treatment of cancer. Lancet Oncol. 2002, 3, 487-497. [CrossRef]

2. Van der Zee, J. Heating the patient: A promising approach? Ann. Oncol. 2002, 13, 1173-1184. [CrossRef] [PubMed] 
3. Horsman, M.R.; Overgaard, J. Hyperthermia: A potent enhancer of radiotherapy. Clin. Oncol. (R Coll. Radiol.) 2007, 19, 418-426. [CrossRef]

4. Engin, K. Biological rationale and clinical experience with hyperthermia. Control Clin. Trials 1996, 17, 316-342. [CrossRef]

5. Oei, A.L.; Vriend, L.E.; Crezee, J.; Franken, N.A.; Krawczyk, P.M. Effects of hyperthermia on DNA repair pathways: One treatment to inhibit them all. Radiat. Oncol. 2015, 10, 165. [CrossRef]

6. Dewey, W.C.; Hopwood, L.E.; Sapareto, S.A.; Gerweck, L.E. Cellular responses to combinations of hyperthermia and radiation. Radiology 1977, 123, 463-474. [CrossRef]

7. Overgaard, J. Effect of hyperthermia on the hypoxic fraction in an experimental mammary carcinoma in vivo. Br. J. Radiol. 1981, 54, 245-249. [CrossRef]

8. $\quad$ Oei, A.L.; Kok, H.P.; Oei, S.B.; Horsman, M.R.; Stalpers, L.J.A.; Franken, N.A.P.; Crezee, J. Molecular and biological rationale of hyperthermia as radio- and chemosensitizer. Adv. Drug Deliv. Rev. 2020, 163-164, 84-97. [CrossRef]

9. Dewhirst, M.W.; Vujaskovic, Z.; Jones, E.; Thrall, D. Re-setting the biologic rationale for thermal therapy. Int. J. Hyperthermia 2005, 21, 779-790. [CrossRef]

10. Lepock, J.R. Role of nuclear protein denaturation and aggregation in thermal radiosensitization. Int. J. Hyperthermia 2004, 20, 115-130. [CrossRef]

11. Calderwood, S.K.; Theriault, J.R.; Gong, J. How is the immune response affected by hyperthermia and heat shock proteins? Int. J. Hyperthermia 2005, 21, 713-716. [CrossRef] [PubMed]

12. Repasky, E.A.; Evans, S.S.; Dewhirst, M.W. Temperature matters! And why it should matter to tumor immunologists. Cancer Immunol. Res. 2013, 1, 210-216. [CrossRef] [PubMed]

13. Mukhopadhaya, A.; Mendecki, J.; Dong, X.; Liu, L.; Kalnicki, S.; Garg, M.; Alfieri, A.; Guha, C. Localized hyperthermia combined with intratumoral dendritic cells induces systemic antitumor immunity. Cancer Res. 2007, 67, 7798-7806. [CrossRef] [PubMed]

14. Frey, B.; Weiss, E.M.; Rubner, Y.; Wunderlich, R.; Ott, O.J.; Sauer, R.; Fietkau, R.; Gaipl, U.S. Old and new facts about hyperthermiainduced modulations of the immune system. Int. J. Hyperthermia 2012, 28, 528-542. [CrossRef] [PubMed]

15. Overgaard, J. Simultaneous and sequential hyperthermia and radiation treatment of an experimental tumor and its surrounding normal tissue in vivo. Int. J. Radiat. Oncol. Biol. Phys. 1980, 6, 1507-1517. [CrossRef]

16. Henle, K.J.; Leeper, D.B. Interaction of hyperthermia and radiation in CHO cells: Recovery kinetics. Radiat. Res. 1976, 66, 505-518. [CrossRef]

17. Overgaard, J.; Suit, H.D. Time-temperature relationship th hyperthermic treatment of malignant and normal tissue in vivo. Cancer Res. 1979, 39, 3248-3253.

18. Nielsen, O.S.; Overgaard, J.; Kamura, T. Influence of thermotolerance on the interaction between hyperthermia and radiation in a solid tumour in vivo. Br. J. Radiol. 1983, 56, 267-273. [CrossRef]

19. Roizin-Towle, L.; Pirro, J.P. The response of human and rodent cells to hyperthermia. Int. J. Radiat. Oncol. Biol. Phys. 1991, 20, 751-756. [CrossRef]

20. Dahl, O.; Mella, O. Effect of timing and sequence of hyperthermia and cyclophosphamide on a neurogenic rat tumor (BT4A) in vivo. Cancer 1983, 52, 983-987. [CrossRef]

21. Sapareto, S.A.; Dewey, W.C. Thermal dose determination in cancer therapy. Int. J. Radiat. Oncol. Biol. Phys. 1984, 10, 787-800. [CrossRef]

22. Van Leeuwen, C.M.; Oei, A.L.; Chin, K.; Crezee, J.; Bel, A.; Westermann, A.M.; Buist, M.R.; Franken, N.A.P.; Stalpers, L.J.A.; Kok, H.P. A short time interval between radiotherapy and hyperthermia reduces in-field recurrence and mortality in women with advanced cervical cancer. Radiat. Oncol. 2017, 12, 75. [CrossRef] [PubMed]

23. Overgaard, J. Influence of sequence and interval on the biological response to combined hyperthermia and radiation. Natl. Cancer Inst. Monogr. 1982, 61, 325-332. [PubMed]

24. Kapp, D.S.; Petersen, I.A.; Cox, R.S.; Hahn, G.M.; Fessenden, P.; Prionas, S.D.; Lee, E.R.; Meyer, J.L.; Samulski, T.V.; Bagshaw, M.A Two or six hyperthermia treatments as an adjunct to radiation therapy yield similar tumor responses: Results of a randomized trial. Int. J. Radiat. Oncol. Biol. Phys. 1990, 19, 1481-1495. [CrossRef]

25. Arcangeli, G.; Nervi, C.; Cividalli, A.; Lovisolo, G.A. Problem of sequence and fractionation in the clinical application of combined heat and radiation. Cancer Res. 1984, 44, 4857s-4863s.

26. Gerweck, L.E.; Gillette, E.L.; Dewey, W.C. Effect of heat and radiation on synchronous Chinese hamster cells: Killing and repair. Radiat. Res. 1975, 64, 611-623. [CrossRef]

27. Pauwels, B.; Korst, A.E.; Lardon, F.; Vermorken, J.B. Combined modality therapy of gemcitabine and radiation. Oncologist 2005, 10, 34-51. [CrossRef]

28. Ohtsubo, T.; Saito, H.; Tanaka, N.; Matsumoto, H.; Sugimoto, C.; Saito, T.; Hayashi, S.; Kano, E. Enhancement of cisplatin sensitivity and platinum uptake by 40 degrees $C$ hyperthermia in resistant cells. Cancer Lett. 1997, 119, 47-52. [CrossRef]

29. Oleson, J.R.; Sim, D.A.; Manning, M.R. Analysis of prognostic variables in hyperthermia treatment of 161 patients. Int. J. Radiat. Oncol. Biol. Phys. 1984, 10, 2231-2239. [CrossRef]

30. Cox, R.S.; Kapp, D.S. Correlation of thermal parameters with outcome in combined radiation therapy-hyperthermia trials. Int. J. Hyperthermia 1992, 8, 719-732. [CrossRef]

31. Dewhirst, M.W.; Sim, D.A. The utility of thermal dose as a predictor of tumor and normal tissue responses to combined radiation and hyperthermia. Cancer Res. 1984, 44, 4772s-4780s. [PubMed] 
32. Leopold, K.A.; Dewhirst, M.; Samulski, T.; Harrelson, J.; Tucker, J.A.; George, S.L.; Dodge, R.K.; Grant, W.; Clegg, S.; Prosnitz, L.R.; et al. Relationships among tumor temperature, treatment time, and histopathological outcome using preoperative hyperthermia with radiation in soft tissue sarcomas. Int. J. Radiat. Oncol. Biol. Phys. 1992, 22, 989-998. [CrossRef]

33. Kroesen, M.; Mulder, H.T.; van Holthe, J.M.L.; Aangeenbrug, A.A.; Mens, J.W.M.; van Doorn, H.C.; Paulides, M.M.; Oomen-de Hoop, E.; Vernhout, R.M.; Lutgens, L.C.; et al. The Effect of the Time Interval Between Radiation and Hyperthermia on Clinical Outcome in 400 Locally Advanced Cervical Carcinoma Patients. Front. Oncol. 2019, 9, 134. [CrossRef] [PubMed]

34. Crezee, J.; Oei, A.L.; Franken, N.A.P.; Stalpers, L.J.A.; Kok, H.P. Response: Commentary: The Impact of the Time Interval Between Radiation and Hyperthermia on Clinical Outcome in Patients With Locally Advanced Cervical Cancer. Front. Oncol. 2020, 10, 528. [CrossRef] [PubMed]

35. Jones, E.L.; Oleson, J.R.; Prosnitz, L.R.; Samulski, T.V.; Vujaskovic, Z.; Yu, D.; Sanders, L.L.; Dewhirst, M.W. Randomized trial of hyperthermia and radiation for superficial tumors. J. Clin. Oncol. 2005, 23, 3079-3085. [CrossRef]

36. Franckena, M.; Fatehi, D.; de Bruijne, M.; Canters, R.A.; van Norden, Y.; Mens, J.W.; van Rhoon, G.C.; van der Zee, J. Hyperthermia dose-effect relationship in 420 patients with cervical cancer treated with combined radiotherapy and hyperthermia. Eur. J. Cancer 2009, 45, 1969-1978. [CrossRef]

37. Kapp, D.S.; Cox, R.S. Thermal treatment parameters are most predictive of outcome in patients with single tumor nodules per treatment field in recurrent adenocarcinoma of the breast. Int. J. Radiat. Oncol. Biol. Phys. 1995, 33, 887-899. [CrossRef]

38. Oleson, J.R.; Samulski, T.V.; Leopold, K.A.; Clegg, S.T.; Dewhirst, M.W.; Dodge, R.K.; George, S.L. Sensitivity of hyperthermia trial outcomes to temperature and time: Implications for thermal goals of treatment. Int. J. Radiat. Oncol. Biol. Phys. 1993, 25, 289-297. [CrossRef]

39. Sherar, M.; Liu, F.F.; Pintilie, M.; Levin, W.; Hunt, J.; Hill, R.; Hand, J.; Vernon, C.; van Rhoon, G.; van der Zee, J.; et al. Relationship between thermal dose and outcome in thermoradiotherapy treatments for superficial recurrences of breast cancer: Data from a phase III trial. Int. J. Radiat. Oncol. Biol. Phys. 1997, 39, 371-380. [CrossRef]

40. Leopold, K.A.; Dewhirst, M.W.; Samulski, T.V.; Dodge, R.K.; George, S.L.; Blivin, J.L.; Prosnitz, L.R.; Oleson, J.R. Cumulative minutes with T90 greater than Tempindex is predictive of response of superficial malignancies to hyperthermia and radiation. Int. J. Radiat. Oncol. Biol. Phys. 1993, 25, 841-847. [CrossRef]

41. Dinges, S.; Harder, C.; Wurm, R.; Buchali, A.; Blohmer, J.; Gellermann, J.; Wust, P.; Randow, H.; Budach, V. Combined treatment of inoperable carcinomas of the uterine cervix with radiotherapy and regional hyperthermia. Results of a phase II trial. Strahlenther. Onkol. 1998, 174, 517-521. [CrossRef] [PubMed]

42. Bakker, A.; van der Zee, J.; van Tienhoven, G.; Kok, H.P.; Rasch, C.R.N.; Crezee, H. Temperature and thermal dose during radiotherapy and hyperthermia for recurrent breast cancer are related to clinical outcome and thermal toxicity: A systematic review. Int. J. Hyperthermia 2019, 36, 1024-1039. [CrossRef]

43. Trefná, H.D.; Crezee, H.; Schmidt, M.; Marder, D.; Lamprecht, U.; Ehmann, M.; Hartmann, J.; Nadobny, J.; Gellermann, J.; van Holthe, N.; et al. Quality assurance guidelines for superficial hyperthermia clinical trials: I. Clinical requirements. Int. J. Hyperthermia 2017, 33, 471-482. [CrossRef]

44. Paulides, M.M.; Dobsicek Trefna, H.; Curto, S.; Rodrigues, D.B. Recent technological advancements in radiofrequency- and microwave-mediated hyperthermia for enhancing drug delivery. Adv. Drug Deliv. Rev. 2020, 163-164, 3-18. [CrossRef] [PubMed]

45. Kok, H.P.; Crezee, J. A comparison of the heating characteristics of capacitive and radiative superficial hyperthermia. Int. J. Hyperthermia 2017, 33, 378-386. [CrossRef] [PubMed]

46. Bruggmoser, G.; Bauchowitz, S.; Canters, R.; Crezee, H.; Ehmann, M.; Gellermann, J.; Lamprecht, U.; Lomax, N.; Messmer, M.B.; Ott, O.; et al. Guideline for the clinical application, documentation and analysis of clinical studies for regional deep hyperthermia: Quality management in regional deep hyperthermia. Strahlenther. Onkol. 2012, 188 (Suppl. 2), 198-211. [CrossRef]

47. Dobšíček Trefná, H.; Crezee, J.; Schmidt, M.; Marder, D.; Lamprecht, U.; Ehmann, M.; Nadobny, J.; Hartmann, J.; Lomax, N.; Abdel-Rahman, S.; et al. Quality assurance guidelines for superficial hyperthermia clinical trials: II. Technical requirements for heating devices. Strahlenther. Onkol. 2017, 193, 351-366. [CrossRef]

48. Kroeze, H.; Kokubo, M.; Kamer, J.B.V.D.; Leeuw, A.A.C.D.; Kikuchi, M.; Hiraoka, M.; Lagendijk, J.J.W. Comparison of a Capacitive and a Cavity Slot Radiative Applicator for Regional Hyperthermia. Therm. Med. (Jpn. J. Hyperthermic Oncol.) 2002, 18, 75-91. [CrossRef]

49. Datta, N.R.; Marder, D.; Datta, S.; Meister, A.; Puric, E.; Stutz, E.; Rogers, S.; Eberle, B.; Timm, O.; Staruch, M.; et al. Quantification of thermal dose in moderate clinical hyperthermia with radiotherapy: A relook using temperature-time area under the curve (AUC). Int. J. Hyperthermia 2021, 38, 296-307. [CrossRef]

50. Fatehi, D.; van der Zee, J.; Notenboom, A.; van Rhoon, G.C. Comparison of intratumor and intraluminal temperatures during locoregional deep hyperthermia of pelvic tumors. Strahlenther. Onkol. 2007, 183, 479-486. [CrossRef]

51. Fatehi, D.; de Bruijne, M.; van der Zee, J.; van Rhoon, G.C. RHyThM, a tool for analysis of PDOS formatted hyperthermia treatment data generated by the BSD2000/3D system. Int. J. Hyperthermia 2006, 22, 173-184. [CrossRef] [PubMed]

52. Overgaard, J. Formula to estimate the thermal enhancement ratio of a single simultaneous hyperthermia and radiation treatment. Acta Radiol. Oncol. 1984, 23, 135-139. [CrossRef] [PubMed]

53. Song, C.W.; Park, H.; Griffin, R.J. Improvement of tumor oxygenation by mild hyperthermia. Radiat. Res. 2001, 155, 515-528. [CrossRef] 
54. Overgaard, J.; Radacic, M.M.; Grau, C. Interaction of hyperthermia and cis-diamminedichloroplatinum(II) alone or combined with radiation in a C3H mammary carcinoma in vivo. Cancer Res. 1991, 51, 707-711. [PubMed]

55. Lindegaard, J.C.; Radacic, M.; Khalil, A.A.; Horsman, M.R.; Overgaard, J. Cisplatin and hyperthermia treatment of a C3H mammary carcinoma in vivo. Importance of sequence, interval, drug dose, and temperature. Acta Oncol. 1992, 31, 347-351. [CrossRef] [PubMed]

56. Hintzsche, H.; Riese, T.; Stopper, H. Hyperthermia-induced micronucleus formation in a human keratinocyte cell line. Mutat. Res. 2012, 738-739, 71-74. [CrossRef]

57. Urano, M.; Ling, C.C. Thermal enhancement of melphalan and oxaliplatin cytotoxicity in vitro. Int. J. Hyperthermia 2002, 18, 307-315. [CrossRef]

58. Kampinga, H.H.; Dynlacht, J.R.; Dikomey, E. Mechanism of radiosensitization by hyperthermia $(>$ or $=43$ degrees C) as derived from studies with DNA repair defective mutant cell lines. Int. J. Hyperthermia 2004, 20, 131-139. [CrossRef]

59. Kampinga, H.H. Cell biological effects of hyperthermia alone or combined with radiation or drugs: A short introduction to newcomers in the field. Int. J. Hyperthermia 2006, 22, 191-196. [CrossRef]

60. Ohtsubo, T.; Chang, S.W.; Tsuji, K.; Picha, P.; Saito, H.; Kano, E. Effects of cis-diamminedichloroplatinum (CDDP) and cisdiammine (1,1-cyclobutanedicarboxylate) platinum (CBDCA) on thermotolerance development and thermosensitivity of the thermotolerant cells. Int. J. Hyperthermia 1990, 6, 1031-1039. [CrossRef]

61. Van der Zee, J.; Peer-Valstar, J.N.; Rietveld, P.J.; de Graaf-Strukowska, L.; van Rhoon, G.C. Practical limitations of interstitial thermometry during deep hyperthermia. Int. J. Radiat. Oncol. Biol. Phys. 1998, 40, 1205-1212. [CrossRef]

62. Oleson, J.R. Eugene Robertson Special Lecture. Hyperthermia from the clinic to the laboratory: A hypothesis. Int. J. Hyperthermia 1995, 11, 315-322. [CrossRef] [PubMed]

63. Sapareto, S.A.; Hopwood, L.E.; Dewey, W.C.; Raju, M.R.; Gray, J.W. Effects of hyperthermia on survival and progression of Chinese hamster ovary cells. Cancer Res. 1978, 38, 393-400. [PubMed]

64. Bing, C.; Patel, P.; Staruch, R.M.; Shaikh, S.; Nofiele, J.; Wodzak Staruch, M.; Szczepanski, D.; Williams, N.S.; Laetsch, T.; Chopra, R. Longer heating duration increases localized doxorubicin deposition and therapeutic index in Vx2 tumors using MR-HIFU mild hyperthermia and thermosensitive liposomal doxorubicin. Int. J. Hyperthermia 2019, 36, 196-203. [CrossRef]

65. Bhuyan, B.K.; Day, K.J.; Edgerton, C.E.; Ogunbase, O. Sensitivity of different cell lines and of different phases in the cell cycle to hyperthermia. Cancer Res. 1977, 37, 3780-3784. [PubMed]

66. Dewey, W.C. Arrhenius relationships from the molecule and cell to the clinic. Int. J. Hyperthermia 2009, 25, 3-20. [CrossRef]

67. Law, M.P. Induced thermal resistance in the mouse ear: The relationship between heating time and temperature. Int. J. Radiat. Biol. Relat. Stud. Phys. Chem. Med. 1979, 35, 481-485. [CrossRef]

68. Li, G.C.; Mivechi, N.F.; Weitzel, G. Heat shock proteins, thermotolerance, and their relevance to clinical hyperthermia. Int. J. Hyperthermia 1995, 11, 459-488. [CrossRef]

69. Field, S.B.; Morris, C.C. The relationship between heating time and temperature: Its relevance to clinical hyperthermia. Radiother. Oncol. 1983, 1, 179-186. [CrossRef]

70. Dewhirst, M.W.; Viglianti, B.L.; Lora-Michiels, M.; Hanson, M.; Hoopes, P.J. Basic principles of thermal dosimetry and thermal thresholds for tissue damage from hyperthermia. Int. J. Hyperthermia 2003, 19, 267-294. [CrossRef]

71. Nielsen, O.S. Fractionated hyperthermia and thermotolerance. Experimental studies on heat-induced resistance in tumour cells treated with hyperthermia alone or in combination with radiotherapy. Dan. Med. Bull. 1984, 31, 376-390. [PubMed]

72. Ben-Hur, E.; Elkind, M.M.; Bronk, B.V. Thermally enhanced radioresponse of cultured Chinese hamster cells: Inhibition of repair of sublethal damage and enhancement of lethal damage. Radiat. Res. 1974, 58, 38-51. [CrossRef] [PubMed]

73. Van Rhoon, G.C.; Samaras, T.; Yarmolenko, P.S.; Dewhirst, M.W.; Neufeld, E.; Kuster, N. CEM $43^{\circ} \mathrm{C}$ thermal dose thresholds: A potential guide for magnetic resonance radiofrequency exposure levels? Eur. Radiol. 2013, 23, 2215-2227. [CrossRef] [PubMed]

74. Yarmolenko, P.S.; Moon, E.J.; Landon, C.; Manzoor, A.; Hochman, D.W.; Viglianti, B.L.; Dewhirst, M.W. Thresholds for thermal damage to normal tissues: An update. Int. J. Hyperthermia 2011, 27, 320-343. [CrossRef]

75. Thrall, D.E.; LaRue, S.M.; Yu, D.; Samulski, T.; Sanders, L.; Case, B.; Rosner, G.; Azuma, C.; Poulson, J.; Pruitt, A.F.; et al. Thermal dose is related to duration of local control in canine sarcomas treated with thermoradiotherapy. Clin. Cancer Res. 2005, 11, 5206-5214. [CrossRef]

76. Kossatz, S.; Ludwig, R.; Dähring, H.; Ettelt, V.; Rimkus, G.; Marciello, M.; Salas, G.; Patel, V.; Teran, F.J.; Hilger, I. High therapeutic efficiency of magnetic hyperthermia in xenograft models achieved with moderate temperature dosages in the tumor area. Pharm. Res. 2014, 31, 3274-3288. [CrossRef]

77. Dewhirst, M.W.; Sim, D.A.; Sapareto, S.; Connor, W.G. Importance of minimum tumor temperature in determining early and long-term responses of spontaneous canine and feline tumors to heat and radiation. Cancer Res. 1984, 44, 43-50.

78. Van Rhoon, G.C. Is CEM43 still a relevant thermal dose parameter for hyperthermia treatment monitoring? Int. J. Hyperthermia 2016, 32, 50-62. [CrossRef]

79. Gerner, E.W.; Boone, R.; Connor, W.G.; Hicks, J.A.; Boone, M.L. A transient thermotolerant survival response produced by single thermal doses in HeLa cells. Cancer Res. 1976, 36, 1035-1040.

80. Li, Z.; Sun, Q.; Huang, X.; Zhang, J.; Hao, J.; Li, Y.; Zhang, S. The Efficacy of Radiofrequency Hyperthermia Combined with Chemotherapy in the Treatment of Advanced Ovarian Cancer. Open Med. 2018, 13, 83-89. [CrossRef] 
81. Kamura, T.; Nielsen, O.S.; Overgaard, J.; Andersen, A.H. Development of thermotolerance during fractionated hyperthermia in a solid tumor in vivo. Cancer Res. 1982, 42, 1744-1748.

82. Zywietz, F.; Reeker, W.; Kochs, E. Changes in tumor oxygenation during a combined treatment with fractionated irradiation and hyperthermia: An experimental study. Int. J. Radiat. Oncol. Biol. Phys. 1997, 37, 155-162. [CrossRef]

83. Nah, B.S.; Choi, I.B.; Oh, W.Y.; Osborn, J.L.; Song, C.W. Vascular thermal adaptation in tumors and normal tissue in rats. Int. J. Radiat. Oncol. Biol. Phys. 1996, 35, 95-101. [CrossRef]

84. Overgaard, J.; Nielsen, O.S. The importance of thermotolerance for the clinical treatment with hyperthermia. Radiother. Oncol. 1983, 1, 167-178. [CrossRef]

85. Mei, X.; Ten Cate, R.; van Leeuwen, C.M.; Rodermond, H.M.; de Leeuw, L.; Dimitrakopoulou, D.; Stalpers, L.J.A.; Crezee, J.; Kok, H.P.; Franken, N.A.P.; et al. Radiosensitization by Hyperthermia: The Effects of Temperature, Sequence, and Time Interval in Cervical Cell Lines. Cancers 2020, 12, 582. [CrossRef] [PubMed]

86. Notter, M.; Piazena, H.; Vaupel, P. Hypofractionated re-irradiation of large-sized recurrent breast cancer with thermographycontrolled, contact-free water-filtered infra-red-A hyperthermia: A retrospective study of 73 patients. Int. J. Hyperthermia 2017, 33, 227-236. [CrossRef] [PubMed]

87. Hurwitz, M.; Stauffer, P. Hyperthermia, radiation and chemotherapy: The role of heat in multidisciplinary cancer care. Semin. Oncol. 2014, 41, 714-729. [CrossRef]

88. Joschko, M.A.; Webster, L.K.; Groves, J.; Yuen, K.; Palatsides, M.; Ball, D.L.; Millward, M.J. Enhancement of radiation-induced regrowth delay by gemcitabine in a human tumor xenograft model. Radiat. Oncol. Investig. 1997, 5, 62-71. [CrossRef]

89. Van Bree, C.; Beumer, C.; Rodermond, H.M.; Haveman, J.; Bakker, P.J. Effectiveness of 2',2'difluorodeoxycytidine (Gemcitabine) combined with hyperthermia in rat R-1 rhabdomyosarcoma in vitro and in vivo. Int. J. Hyperthermia 1999, 15, 549-556.

90. Li, G.C.; Kal, H.B. Effect of hyperthermia on the radiation response of two mammalian cell lines. Eur. J. Cancer 1977, 13, 65-69. [CrossRef]

91. Chen, H.; Ma, G.; Wang, X.; Zhou, W.; Wang, S. Time interval after heat stress plays an important role in the combination therapy of hyperthermia and cancer chemotherapy agents. Int. J. Hyperthermia 2020, 37, 254-255. [CrossRef] [PubMed]

92. Mitsumori, M.; Zeng, Z.F.; Oliynychenko, P.; Park, J.H.; Choi, I.B.; Tatsuzaki, H.; Tanaka, Y.; Hiraoka, M. Regional hyperthermia combined with radiotherapy for locally advanced non-small cell lung cancers: A multi-institutional prospective randomized trial of the International Atomic Energy Agency. Int. J. Clin. Oncol. 2007, 12, 192-198. [CrossRef] [PubMed]

93. Wust, P.; Gellermann, J.; Harder, C.; Tilly, W.; Rau, B.; Dinges, S.; Schlag, P.; Budach, V.; Felix, R. Rationale for using invasive thermometry for regional hyperthermia of pelvic tumors. Int. J. Radiat. Oncol. Biol. Phys. 1998, 41, 1129-1137. [CrossRef]

94. Ohguri, T.; Imada, H.; Yahara, K.; Morioka, T.; Nakano, K.; Terashima, H.; Korogi, Y. Radiotherapy with 8-MHz radiofrequencycapacitive regional hyperthermia for stage III non-small-cell lung cancer: The radiofrequency-output power correlates with the intraesophageal temperature and clinical outcomes. Int. J. Radiat. Oncol. Biol. Phys. 2009, 73, 128-135. [CrossRef] [PubMed]

95. Ohguri, T.; Yahara, K.; Moon, S.D.; Yamaguchi, S.; Imada, H.; Terashima, H.; Korogi, Y. Deep regional hyperthermia for the whole thoracic region using $8 \mathrm{MHz}$ radiofrequency-capacitive heating device: Relationship between the radiofrequency-output power and the intra-oesophageal temperature and predictive factors for a good heating in 59 patients. Int. J. Hyperthermia 2011, 27, 20-26. [PubMed]

96. Chi, M.S.; Yang, K.L.; Chang, Y.C.; Ko, H.L.; Lin, Y.H.; Huang, S.C.; Huang, Y.Y.; Liao, K.W.; Kondo, M.; Chi, K.H. Comparing the Effectiveness of Combined External Beam Radiation and Hyperthermia Versus External Beam Radiation Alone in Treating Patients With Painful Bony Metastases: A Phase 3 Prospective, Randomized, Controlled Trial. Int. J. Radiat. Oncol. Biol. Phys. 2018, 100, 78-87. [CrossRef]

97. Nishimura, Y.; Hiraoka, M.; Akuta, K.; Jo, S.; Nagata, Y.; Masunaga, S.; Takahashi, M.; Abe, M. Hyperthermia combined with radiation therapy for primarily unresectable and recurrent colorectal cancer. Int. J. Radiat. Oncol. Biol. Phys. 1992, 23, 759-768. [CrossRef]

98. Engin, K.; Tupchong, L.; Moylan, D.J.; Alexander, G.A.; Waterman, F.M.; Komarnicky, L.; Nerlinger, R.E.; Leeper, D.B. Randomized trial of one versus two adjuvant hyperthermia treatments per week in patients with superficial tumours. Int. J. Hyperthermia 1993, 9, 327-340. [CrossRef]

99. Tilly, W.; Gellermann, J.; Graf, R.; Hildebrandt, B.; Weissbach, L.; Budach, V.; Felix, R.; Wust, P. Regional hyperthermia in conjunction with definitive radiotherapy against recurrent or locally advanced prostate cancer T3 pN0 M0. Strahlenther. Onkol. 2005, 181, 35-41. [CrossRef]

100. Masunaga, S.I.; Hiraoka, M.; Akuta, K.; Nishimura, Y.; Nagata, Y.; Jo, S.; Takahashi, M.; Abe, M.; Terachi, T.; Oishi, K.; et al. Phase I/II trial of preoperative thermoradiotherapy in the treatment of urinary bladder cancer. Int. J. Hyperthermia 1994, 10, 31-40. [CrossRef]

101. Valdagni, R.; Liu, F.F.; Kapp, D.S. Important prognostic factors influencing outcome of combined radiation and hyperthermia. Int. J. Radiat. Oncol. Biol. Phys. 1988, 15, 959-972. [CrossRef]

102. Kroesen, M.; Mulder, H.T.; van Holthe, J.M.L.; Aangeenbrug, A.A.; Mens, J.W.M.; van Doorn, H.C.; Paulides, M.M.; Oomen-de Hoop, E.; Vernhout, R.M.; Lutgens, L.C.; et al. Confirmation of thermal dose as a predictor of local control in cervical carcinoma patients treated with state-of-the-art radiation therapy and hyperthermia. Radiother. Oncol. 2019, 140, 150-158. [CrossRef] [PubMed] 
103. Valdagni, R.; Amichetti, M. Report of long-term follow-up in a randomized trial comparing radiation therapy and radiation therapy plus hyperthermia to metastatic lymph nodes in stage IV head and neck patients. Int. J. Radiat. Oncol. Biol. Phys. 1994, 28, 163-169. [CrossRef]

104. Van der Zee, J.; González González, D.; van Rhoon, G.C.; van Dijk, J.D.; van Putten, W.L.; Hart, A.A.; Dutch Deep Hyperthermia Group. Comparison of radiotherapy alone with radiotherapy plus hyperthermia in locally advanced pelvic tumours: A prospective, randomised, multicentre trial. Lancet 2000, 355, 1119-1125. [CrossRef]

105. Harima, Y.; Nagata, K.; Harima, K.; Ostapenko, V.V.; Tanaka, Y.; Sawada, S. A randomized clinical trial of radiation therapy versus thermoradiotherapy in stage IIIB cervical carcinoma. Int. J. Hyperthermia 2001, 17, 97-105. [CrossRef] [PubMed]

106. Berdov, B.A.; Menteshashvili, G.Z. Thermoradiotherapy of patients with locally advanced carcinoma of the rectum. Int. J. Hyperthermia 1990, 6, 881-890. [CrossRef]

107. Maluta, S.; Dall'Oglio, S.; Romano, M.; Marciai, N.; Pioli, F.; Giri, M.G.; Benecchi, P.L.; Comunale, L.; Porcaro, A.B. Conformal radiotherapy plus local hyperthermia in patients affected by locally advanced high risk prostate cancer: Preliminary results of a prospective phase II study. Int. J. Hyperthermia 2007, 23, 451-456. [CrossRef] [PubMed]

108. Anscher, M.S.; Samulski, T.V.; Dodge, R.; Prosnitz, L.R.; Dewhirst, M.W. Combined external beam irradiation and external regional hyperthermia for locally advanced adenocarcinoma of the prostate. Int. J. Radiat. Oncol. Biol. Phys. 1997, 37, 1059-1065. [CrossRef]

109. Gabriele, P.; Amichetti, M.; Orecchia, R.; Valdagni, R. Hyperthermia and radiation therapy for inoperable or recurrent parotid carcinoma. A phase I/II study. Cancer 1995, 75, 908-913. [CrossRef]

110. Maguire, P.D.; Samulski, T.V.; Prosnitz, L.R.; Jones, E.L.; Rosner, G.L.; Powers, B.; Layfield, L.W.; Brizel, D.M.; Scully, S.P.; Harrelson, J.M.; et al. A phase II trial testing the thermal dose parameter CEM43 degrees T90 as a predictor of response in soft tissue sarcomas treated with pre-operative thermoradiotherapy. Int. J. Hyperthermia 2001, 17, 283-290. [CrossRef]

111. Lutgens, L.C.; Koper, P.C.; Jobsen, J.J.; van der Steen-Banasik, E.M.; Creutzberg, C.L.; van den Berg, H.A.; Ottevanger, P.B.; van Rhoon, G.C.; van Doorn, H.C.; Houben, R.; et al. Radiation therapy combined with hyperthermia versus cisplatin for locally advanced cervical cancer: Results of the randomized RADCHOC trial. Radiother. Oncol. 2016, 120, 378-382. [CrossRef] [PubMed]

112. Hurwitz, M.D.; Hansen, J.L.; Prokopios-Davos, S.; Manola, J.; Wang, Q.; Bornstein, B.A.; Hynynen, K.; Kaplan, I.D. Hyperthermia combined with radiation for the treatment of locally advanced prostate cancer: Long-term results from Dana-Farber Cancer Institute study 94-153. Cancer 2011, 117, 510-516. [CrossRef] [PubMed]

113. Vernon, C.C.; Hand, J.W.; Field, S.B.; Machin, D.; Whaley, J.B.; van der Zee, J.; van Putten, W.L.; van Rhoon, G.C.; van Dijk, J.D.; González González, D.; et al. Radiotherapy with or without hyperthermia in the treatment of superficial localized breast cancer: Results from five randomized controlled trials. International Collaborative Hyperthermia Group. Int. J. Radiat. Oncol. Biol. Phys. 1996, 35, 731-744. [PubMed]

114. Datta, N.R.; Bose, A.K.; Kapoor, H.K.; Gupta, S. Head and neck cancers: Results of thermoradiotherapy versus radiotherapy. Int. J. Hyperthermia 1990, 6, 479-486. [CrossRef]

115. Overgaard, J.; Gonzalez Gonzalez, D.; Hulshof, M.C.; Arcangeli, G.; Dahl, O.; Mella, O.; Bentzen, S.M. Randomised trial of hyperthermia as adjuvant to radiotherapy for recurrent or metastatic malignant melanoma. European Society for Hyperthermic Oncology. Lancet 1995, 345, 540-543. [CrossRef]

116. Overgaard, J.; Gonzalez Gonzalez, D.; Hulshof, M.C.; Arcangeli, G.; Dahl, O.; Mella, O.; Bentzen, S.M. Hyperthermia as an adjuvant to radiation therapy of recurrent or metastatic malignant melanoma. A multicentre randomized trial by the European Society for Hyperthermic Oncology. Int. J. Hyperthermia 1996, 12, 3-20. [CrossRef] [PubMed]

117. Kim, B.S.; Chung, H.C.; Seong, J.S.; Suh, C.O.; Kim, G.E. Phase II trial for combined external radiotherapy and hyperthermia for unresectable hepatoma. Cancer Chemother. Pharmacol. 1992, 31, S119-S127. [CrossRef]

118. Overgaard, J.; Overgaard, M.; Hansen, P.V.; von der Maase, H. Some factors of importance in the radiation treatment of malignant melanoma. Radiother. Oncol. 1986, 5, 183-192. [CrossRef]

119. Emami, B.; Perez, C.A.; Konefal, J.; Pilepich, M.V.; Leybovich, L.; Straube, W.; VonGerichten, D.; Hederman, M.A. Thermoradiotherapy of malignant melanoma. Int. J. Hyperthermia 1988, 4, 373-381. [CrossRef]

120. Franckena, M.; Lutgens, L.C.; Koper, P.C.; Kleynen, C.E.; van der Steen-Banasik, E.M.; Jobsen, J.J.; Leer, J.W.; Creutzberg, C.L.; Dielwart, M.F.; van Norden, Y.; et al. Radiotherapy and hyperthermia for treatment of primary locally advanced cervix cancer: Results in 378 patients. Int. J. Radiat. Oncol. Biol. Phys. 2009, 73, 242-250. [CrossRef]

121. Oldenborg, S.; Van Os, R.M.; Van rij, C.M.; Crezee, J.; Van de Kamer, J.B.; Rutgers, E.J.; Geijsen, E.D.; Zum vörde sive vörding, P.J.; Koning, C.C.; Van tienhoven, G. Elective re-irradiation and hyperthermia following resection of persistent locoregional recurrent breast cancer: A retrospective study. Int. J. Hyperthermia 2010, 26, 136-144. [CrossRef] [PubMed]

122. Shen, H.; Li, X.D.; Wu, C.P.; Yin, Y.M.; Wang, R.S.; Shu, Y.Q. The regimen of gemcitabine and cisplatin combined with radio frequency hyperthermia for advanced non-small cell lung cancer: A phase II study. Int. J. Hyperthermia 2011, 27, 27-32. [CrossRef]

123. Issels, R.D.; Lindner, L.H.; Verweij, J.; Wust, P.; Reichardt, P.; Schem, B.C.; Abdel-Rahman, S.; Daugaard, S.; Salat, C.; Wendtner, C.M.; et al. Neo-adjuvant chemotherapy alone or with regional hyperthermia for localised high-risk soft-tissue sarcoma: A randomised phase 3 multicentre study. Lancet Oncol. 2010, 11, 561-570. [CrossRef]

124. Sugimachi, K.; Kuwano, H.; Ide, H.; Toge, T.; Saku, M.; Oshiumi, Y. Chemotherapy combined with or without hyperthermia for patients with oesophageal carcinoma: A prospective randomized trial. Int. J. Hyperthermia 1994, 10, 485-493. [CrossRef] 
125. Colombo, R.; Salonia, A.; Leib, Z.; Pavone-Macaluso, M.; Engelstein, D. Long-term outcomes of a randomized controlled trial comparing thermochemotherapy with mitomycin- $\mathrm{C}$ alone as adjuvant treatment for non-muscle-invasive bladder cancer (NMIBC). BJU Int. 2011, 107, 912-918. [CrossRef] [PubMed]

126. Braun, J.; Hahn, G.M. Enhanced cell killing by bleomycin and 43 degrees hyperthermia and the inhibition of recovery from potentially lethal damage. Cancer Res. 1975, 35, 2921-2927.

127. Zagar, T.M.; Vujaskovic, Z.; Formenti, S.; Rugo, H.; Muggia, F.; O’Connor, B.; Myerson, R.; Stauffer, P.; Hsu, I.C.; Diederich, C.; et al. Two phase I dose-escalation/pharmacokinetics studies of low temperature liposomal doxorubicin (LTLD) and mild local hyperthermia in heavily pretreated patients with local regionally recurrent breast cancer. Int. J. Hyperthermia 2014, 30, 285-294. [CrossRef]

128. Wallner, K.E.; DeGregorio, M.W.; Li, G.C. Hyperthermic potentiation of cis-diamminedichloroplatinum(II) cytotoxicity in Chinese hamster ovary cells resistant to the drug. Cancer Res. 1986, 46, 6242-6245.

129. Magin, R.L.; Sikic, B.I.; Cysyk, R.L. Enhancement of bleomycin activity against Lewis lung tumors in mice by local hyperthermia. Cancer Res. 1979, 39, 3792-3795.

130. Kong, G.; Anyarambhatla, G.; Petros, W.P.; Braun, R.D.; Colvin, O.M.; Needham, D.; Dewhirst, M.W. Efficacy of liposomes and hyperthermia in a human tumor xenograft model: Importance of triggered drug release. Cancer Res. 2000, 60, 6950-6957.

131. Van der Heijden, A.G.; Jansen, C.F.; Verhaegh, G.; O'Donnell M, A.; Schalken, J.A.; Witjes, J.A. The effect of hyperthermia on mitomycin-C induced cytotoxicity in four human bladder cancer cell lines. Eur. Urol. 2004, 46, 670-674. [CrossRef] [PubMed]

132. Van Rhoon, G.C.; Franckena, M.; Ten Hagen, T.L.M. A moderate thermal dose is sufficient for effective free and TSL based thermochemotherapy. Adv. Drug Deliv. Rev. 2020, 163-164, 145-156. [CrossRef] [PubMed]

133. Rietbroek, R.C.; Schilthuis, M.S.; Bakker, P.J.; van Dijk, J.D.; Postma, A.J.; González González, D.; Bakker, A.J.; van der Velden, J.; Helmerhorst, T.J.; Veenhof, C.H. Phase II trial of weekly locoregional hyperthermia and cisplatin in patients with a previously irradiated recurrent carcinoma of the uterine cervix. Cancer 1997, 79, 935-943. [CrossRef]

134. Fiegl, M.; Schlemmer, M.; Wendtner, C.M.; Abdel-Rahman, S.; Fahn, W.; Issels, R.D. Ifosfamide, carboplatin and etoposide (ICE) as second-line regimen alone and in combination with regional hyperthermia is active in chemo-pre-treated advanced soft tissue sarcoma of adults. Int. J. Hyperthermia 2004, 20, 661-670. [CrossRef] [PubMed]

135. De Wit, R.; van der Zee, J.; van der Burg, M.E.; Kruit, W.H.; Logmans, A.; van Rhoon, G.C.; Verweij, J. A phase I/II study of combined weekly systemic cisplatin and locoregional hyperthermia in patients with previously irradiated recurrent carcinoma of the uterine cervix. Br. J. Cancer 1999, 80, 1387-1391. [CrossRef]

136. Alvarez Secord, A.; Jones, E.L.; Hahn, C.A.; Petros, W.P.; Yu, D.; Havrilesky, L.J.; Soper, J.T.; Berchuck, A.; Spasojevic, I.; ClarkePearson, D.L.; et al. Phase I/II trial of intravenous Doxil and whole abdomen hyperthermia in patients with refractory ovarian cancer. Int. J. Hyperthermia 2005, 21, 333-347. [CrossRef]

137. Stahl, R.; Wang, T.; Lindner, L.H.; Abdel-Rahman, S.; Santl, M.; Reiser, M.F.; Issels, R.D. Comparison of radiological and pathohistological response to neoadjuvant chemotherapy combined with regional hyperthermia (RHT) and study of response dependence on the applied thermal parameters in patients with soft tissue sarcomas (STS). Int. J. Hyperthermia 2009, 25, 289-298. [CrossRef]

138. Vujaskovic, Z.; Kim, D.W.; Jones, E.; Lan, L.; McCall, L.; Dewhirst, M.W.; Craciunescu, O.; Stauffer, P.; Liotcheva, V.; Betof, A.; et al. A phase I/II study of neoadjuvant liposomal doxorubicin, paclitaxel, and hyperthermia in locally advanced breast cancer. Int. J. Hyperthermia 2010, 26, 514-521. [CrossRef]

139. Ishikawa, T.; Kokura, S.; Sakamoto, N.; Ando, T.; Imamoto, E.; Hattori, T.; Oyamada, H.; Yoshinami, N.; Sakamoto, M.; Kitagawa, K.; et al. Phase II trial of combined regional hyperthermia and gemcitabine for locally advanced or metastatic pancreatic cancer. Int. J. Hyperthermia 2012, 28, 597-604. [CrossRef]

140. Yang, W.H.; Xie, J.; Lai, Z.Y.; Yang, M.D.; Zhang, G.H.; Li, Y.; Mu, J.B.; Xu, J. Radiofrequency deep hyperthermia combined with chemotherapy in the treatment of advanced non-small cell lung cancer. Chin. Med. J. 2019, 132, 922-927. [CrossRef]

141. Tschoep-Lechner, K.E.; Milani, V.; Berger, F.; Dieterle, N.; Abdel-Rahman, S.; Salat, C.; Issels, R.D. Gemcitabine and cisplatin combined with regional hyperthermia as second-line treatment in patients with gemcitabine-refractory advanced pancreatic cancer. Int. J. Hyperthermia 2013, 29, 8-16. [CrossRef] [PubMed]

142. Monge, O.R.; Rofstad, E.K.; Kaalhus, O. Thermochemotherapy in vivo of a C3H mouse mammary carcinoma: Single fraction heat and drug treatment. Eur. J. Cancer Clin. Oncol. 1988, 24, 1661-1669. [CrossRef]

143. Adachi, S.; Kokura, S.; Okayama, T.; Ishikawa, T.; Takagi, T.; Handa, O.; Naito, Y.; Yoshikawa, T. Effect of hyperthermia combined with gemcitabine on apoptotic cell death in cultured human pancreatic cancer cell lines. Int. J. Hyperthermia 2009, 25, 210-219. [CrossRef] [PubMed]

144. Albregts, M.; Hulshof, M.C.; Zum Vörde Sive Vörding, P.J.; van Lanschot, J.J.; Richel, D.J.; Crezee, H.; Fockens, P.; van Dijk, J.D.; González González, D. A feasibility study in oesophageal carcinoma using deep loco-regional hyperthermia combined with concurrent chemotherapy followed by surgery. Int. J. Hyperthermia 2004, 20, 647-659. [CrossRef] [PubMed]

145. Herman, T.S.; Teicher, B.A.; Jochelson, M.; Clark, J.; Svensson, G.; Coleman, C.N. Rationale for use of local hyperthermia with radiation therapy and selected anticancer drugs in locally advanced human malignancies. Int. J. Hyperthermia 1988, 4, 143-158. [CrossRef] 
146. Herman, T.S.; Jochelson, M.S.; Teicher, B.A.; Scott, P.J.; Hansen, J.; Clark, J.R.; Pfeffer, M.R.; Gelwan, L.E.; Molnar-Griffin, B.J.; Fraser, S.M.; et al. A phase I-II trial of cisplatin, hyperthermia and radiation in patients with locally advanced malignancies. Int. J. Radiat. Oncol. Biol. Phys. 1989, 17, 1273-1279. [CrossRef]

147. Herman, T.S.; Teicher, B.A. Sequencing of trimodality therapy[cis-diamminedichloroplatinum(II)/hyperthermia/radiation] as determined by tumor growth delay and tumor cell survival in the FSaIIC fibrosarcoma. Cancer Res. 1988, 48, $2693-2697$.

148. Ohguri, T.; Imada, H.; Yahara, K.; Narisada, H.; Morioka, T.; Nakano, K.; Korogi, Y. Concurrent chemoradiotherapy with gemcitabine plus regional hyperthermia for locally advanced pancreatic carcinoma: Initial experience. Radiat. Med. 2008, 26, 587-596. [CrossRef]

149. Harima, Y.; Ohguri, T.; Imada, H.; Sakurai, H.; Ohno, T.; Hiraki, Y.; Tuji, K.; Tanaka, M.; Terashima, H. A multicentre randomised clinical trial of chemoradiotherapy plus hyperthermia versus chemoradiotherapy alone in patients with locally advanced cervical cancer. Int. J. Hyperthermia 2016, 32, 801-808. [CrossRef]

150. Westermann, A.M.; Jones, E.L.; Schem, B.C.; van der Steen-Banasik, E.M.; Koper, P.; Mella, O.; Uitterhoeve, A.L.; de Wit, R.; van der Velden, J.; Burger, C.; et al. First results of triple-modality treatment combining radiotherapy, chemotherapy, and hyperthermia for the treatment of patients with stage IIB, III, and IVA cervical carcinoma. Cancer 2005, 104, 763-770. [CrossRef]

151. Asao, T.; Sakurai, H.; Harashima, K.; Yamaguchi, S.; Tsutsumi, S.; Nonaka, T.; Shioya, M.; Nakano, T.; Kuwano, H. The synchronization of chemotherapy to circadian rhythms and irradiation in pre-operative chemoradiation therapy with hyperthermia for local advanced rectal cancer. Int. J. Hyperthermia 2006, 22, 399-406. [CrossRef] [PubMed]

152. Amichetti, M.; Graiff, C.; Fellin, G.; Pani, G.; Bolner, A.; Maluta, S.; Valdagni, R. Cisplatin, hyperthermia, and radiation (trimodal therapy) in patients with locally advanced head and neck tumors: A phase I-II study. Int. J. Radiat. Oncol. Biol. Phys. 1993, 26, 801-807. [CrossRef]

153. Kouloulias, V.E.; Dardoufas, C.E.; Kouvaris, J.R.; Gennatas, C.S.; Polyzos, A.K.; Gogas, H.J.; Sandilos, P.H.; Uzunoglu, N.K.; Malas, E.G.; Vlahos, L.J. Liposomal doxorubicin in conjunction with reirradiation and local hyperthermia treatment in recurrent breast cancer: A phase I/II trial. Clin. Cancer Res. 2002, 8, 374-382.

154. Gani, C.; Lamprecht, U.; Ziegler, A.; Moll, M.; Gellermann, J.; Heinrich, V.; Wenz, S.; Fend, F.; Königsrainer, A.; Bitzer, M.; et al. Deep regional hyperthermia with preoperative radiochemotherapy in locally advanced rectal cancer, a prospective phase II trial. Radiother. Oncol. 2021, 159, 155-160. [CrossRef] [PubMed]

155. Ohguri, T.; Harima, Y.; Imada, H.; Sakurai, H.; Ohno, T.; Hiraki, Y.; Tuji, K.; Tanaka, M.; Terashima, H. Relationships between thermal dose parameters and the efficacy of definitive chemoradiotherapy plus regional hyperthermia in the treatment of locally advanced cervical cancer: Data from a multicentre randomised clinical trial. Int. J. Hyperthermia 2018, 34, 461-468. [CrossRef]

156. Maluta, S.; Schaffer, M.; Pioli, F.; Dall'oglio, S.; Pasetto, S.; Schaffer, P.M.; Weber, B.; Giri, M.G. Regional hyperthermia combined with chemoradiotherapy in primary or recurrent locally advanced pancreatic cancer: An open-label comparative cohort trial. Strahlenther. Onkol. 2011, 187, 619-625. [CrossRef]

157. Barsukov, Y.A.; Gordeyev, S.S.; Tkachev, S.I.; Fedyanin, M.Y.; Perevoshikov, A.G. Phase II study of concomitant chemoradiotherapy with local hyperthermia and metronidazole for locally advanced fixed rectal cancer. Colorectal Dis. 2013, 15, 1107-1114. [CrossRef]

158. Ott, O.J.; Gani, C.; Lindner, L.H.; Schmidt, M.; Lamprecht, U.; Abdel-Rahman, S.; Hinke, A.; Weissmann, T.; Hartmann, A.; Issels, R.D.; et al. Neoadjuvant Chemoradiation Combined with Regional Hyperthermia in Locally Advanced or Recurrent Rectal Cancer. Cancers 2021, 13, 1279. [CrossRef]

159. Rau, B.; Wust, P.; Hohenberger, P.; Löffel, J.; Hünerbein, M.; Below, C.; Gellermann, J.; Speidel, A.; Vogl, T.; Riess, H.; et al. Preoperative hyperthermia combined with radiochemotherapy in locally advanced rectal cancer: A phase II clinical trial. Ann. Surg. 1998, 227, 380-389. [CrossRef]

160. Rau, B.; Wust, P.; Tilly, W.; Gellermann, J.; Harder, C.; Riess, H.; Budach, V.; Felix, R.; Schlag, P.M. Preoperative radiochemotherapy in locally advanced or recurrent rectal cancer: Regional radiofrequency hyperthermia correlates with clinical parameters. Int. J. Radiat. Oncol. Biol. Phys. 2000, 48, 381-391. [CrossRef]

161. Wittlinger, M.; Rödel, C.M.; Weiss, C.; Krause, S.F.; Kühn, R.; Fietkau, R.; Sauer, R.; Ott, O.J. Quadrimodal treatment of highrisk T1 and T2 bladder cancer: Transurethral tumor resection followed by concurrent radiochemotherapy and regional deep hyperthermia. Radiother. Oncol. 2009, 93, 358-363. [CrossRef] [PubMed]

162. Milani, V.; Pazos, M.; Issels, R.D.; Buecklein, V.; Rahman, S.; Tschoep, K.; Schaffer, P.; Wilkowski, R.; Duehmke, E.; Schaffer, M. Radiochemotherapy in combination with regional hyperthermia in preirradiated patients with recurrent rectal cancer. Strahlenther. Onkol. 2008, 184, 163-168. [CrossRef] [PubMed]

163. Zhu, H.; Huo, X.; Chen, L.; Wang, H.; Yu, H. Clinical experience with radio-, chemo- and hyperthermotherapy combined trimodality on locally advanced esophageal cancer. Mol. Clin. Oncol. 2013, 1, 1009-1012. [CrossRef] [PubMed]

164. Gani, C.; Schroeder, C.; Heinrich, V.; Spillner, P.; Lamprecht, U.; Berger, B.; Zips, D. Long-term local control and survival after preoperative radiochemotherapy in combination with deep regional hyperthermia in locally advanced rectal cancer. Int. J. Hyperthermia 2016, 32, 187-192. [CrossRef] [PubMed]

165. Merten, R.; Ott, O.; Haderlein, M.; Bertz, S.; Hartmann, A.; Wullich, B.; Keck, B.; Kühn, R.; Rödel, C.M.; Weiss, C.; et al. Long-Term Experience of Chemoradiotherapy Combined with Deep Regional Hyperthermia for Organ Preservation in High-Risk Bladder Cancer (Ta, Tis, T1, T2). Oncologist 2019, 24, e1341-e1350. [CrossRef] [PubMed] 
166. Van Haaren, P.M.; Hulshof, M.C.; Kok, H.P.; Oldenborg, S.; Geijsen, E.D.; Van Lanschot, J.J.; Crezee, J. Relation between body size and temperatures during locoregional hyperthermia of oesophageal cancer patients. Int. J. Hyperthermia 2008, 24, 663-674. [CrossRef]

167. Curto, S.; Aklan, B.; Mulder, T.; Mils, O.; Schmidt, M.; Lamprecht, U.; Peller, M.; Wessalowski, R.; Lindner, L.H.; Fietkau, R.; et al. Quantitative, Multi-institutional Evaluation of MR Thermometry Accuracy for Deep-Pelvic MR-Hyperthermia Systems Operating in Multi-vendor MR-systems Using a New Anthropomorphic Phantom. Cancers 2019, 11, 1709. [CrossRef]

168. Winter, L.; Oberacker, E.; Paul, K.; Ji, Y.; Oezerdem, C.; Ghadjar, P.; Thieme, A.; Budach, V.; Wust, P.; Niendorf, T. Magnetic resonance thermometry: Methodology, pitfalls and practical solutions. Int. J. Hyperthermia 2016, 32, 63-75. [CrossRef]

169. Adibzadeh, F.; Sumser, K.; Curto, S.; Yeo, D.T.B.; Shishegar, A.A.; Paulides, M.M. Systematic review of pre-clinical and clinical devices for magnetic resonance-guided radiofrequency hyperthermia. Int. J. Hyperthermia 2020, 37, 15-27. [CrossRef]

170. Gellermann, J.; Faehling, H.; Mielec, M.; Cho, C.H.; Budach, V.; Wust, P. Image artifacts during MRT hybrid hyperthermia-causes and elimination. Int. J. Hyperthermia 2008, 24, 327-335. [CrossRef]

171. Ishihara, Y.; Calderon, A.; Watanabe, H.; Okamoto, K.; Suzuki, Y.; Kuroda, K.; Suzuki, Y. A precise and fast temperature mapping using water proton chemical shift. Magn. Reson. Med. 1995, 34, 814-823. [CrossRef] [PubMed]

172. Le Bihan, D.; Delannoy, J.; Levin, R.L. Temperature mapping with MR imaging of molecular diffusion: Application to hyperthermia. Radiology 1989, 171, 853-857. [CrossRef] [PubMed]

173. Gellermann, J.; Wlodarczyk, W.; Feussner, A.; Fähling, H.; Nadobny, J.; Hildebrandt, B.; Felix, R.; Wust, P. Methods and potentials of magnetic resonance imaging for monitoring radiofrequency hyperthermia in a hybrid system. Int. J. Hyperthermia 2005, 21, 497-513. [CrossRef] [PubMed]

174. Lüdemann, L.; Wlodarczyk, W.; Nadobny, J.; Weihrauch, M.; Gellermann, J.; Wust, P. Non-invasive magnetic resonance thermography during regional hyperthermia. Int. J. Hyperthermia 2010, 26, 273-282. [CrossRef]

175. Gellermann, J.; Wlodarczyk, W.; Ganter, H.; Nadobny, J.; Fähling, H.; Seebass, M.; Felix, R.; Wust, P. A practical approach to thermography in a hyperthermia/magnetic resonance hybrid system: Validation in a heterogeneous phantom. Int. J. Radiat. Oncol. Biol. Phys. 2005, 61, 267-277. [CrossRef]

176. Gellermann, J.; Hildebrandt, B.; Issels, R.; Ganter, H.; Wlodarczyk, W.; Budach, V.; Felix, R.; Tunn, P.U.; Reichardt, P.; Wust, P. Noninvasive magnetic resonance thermography of soft tissue sarcomas during regional hyperthermia: Correlation with response and direct thermometry. Cancer 2006, 107, 1373-1382. [CrossRef]

177. Gellermann, J.; Wlodarczyk, W.; Hildebrandt, B.; Ganter, H.; Nicolau, A.; Rau, B.; Tilly, W.; Fähling, H.; Nadobny, J.; Felix, R.; et al. Noninvasive magnetic resonance thermography of recurrent rectal carcinoma in a 1.5 Tesla hybrid system. Cancer Res. 2005, 65, 5872-5880. [CrossRef]

178. Poni, R.; Neufeld, E.; Capstick, M.; Bodis, S.; Samaras, T.; Kuster, N. Feasibility of Temperature Control by Electrical Impedance Tomography in Hyperthermia. Cancers 2021, 13, 3297. [CrossRef]

179. Esrick, M.A.; McRae, D.A. The effect of hyperthermia-induced tissue conductivity changes on electrical impedance temperature mapping. Phys. Med. Biol. 1994, 39, 133-144. [CrossRef]

180. Paulsen, K.D.; Moskowitz, M.J.; Ryan, T.P.; Mitchell, S.E.; Hoopes, P.J. Initial in vivo experience with EIT as a thermal estimator during hyperthermia. Int. J. Hyperthermia 1996, 12, 573-591. [CrossRef]

181. Nguyen, D.M.; Andersen, T.; Qian, P.; Barry, T.; McEwan, A. Electrical Impedance Tomography for monitoring cardiac radiofrequency ablation: A scoping review of an emerging technology. Med. Eng. Phys. 2020, 84, 36-50. [CrossRef] [PubMed] 